\title{
Sobolev stability of Prandtl expansions for the steady Navier-Stokes equations
}

\author{
*David Gerard-Varet †Yasunori Maekawa
}

November 8, 2018

\begin{abstract}
We show the $H^{1}$ stability of shear flows of Prandtl type: $U^{\nu}=\left(U_{s}(y / \sqrt{\nu}), 0\right)$, in the steady two-dimensional Navier-Stokes equations, under the natural assumptions that $U_{s}(Y)>0$ for $Y>0, U_{s}(0)=0$, and $U_{s}^{\prime}(0)>0$. Our result is in sharp contrast with the unsteady ones, in which at most Gevrey stability can be obtained, even under global monotonicity and concavity hypotheses. It provides the first positive answer to the inviscid limit problem in Sobolev regularity for a non-trivial class of steady Navier-Stokes flows with no-slip boundary condition.
\end{abstract}

\section{Introduction and main result}

Our concern in this paper is the vanishing viscosity limit of the two-dimensional steady Navier-Stokes equations:

$$
\left\{\begin{array}{l}
v^{\nu} \cdot \nabla v^{\nu}-\nu \Delta v^{\nu}+\nabla q^{\nu}=g^{\nu}, \quad(x, y) \in \mathbb{T}_{\kappa} \times \mathbb{R}_{+}, \\
\operatorname{div} v^{\nu}=0, \quad(x, y) \in \mathbb{T}_{\kappa} \times \mathbb{R}_{+}, \\
\left.v^{\nu}\right|_{y=0}=0 .
\end{array}\right.
$$

Here $\mathbb{T}_{\kappa}=\mathbb{R} /(2 \pi \kappa) \mathbb{Z}, \kappa>0$, is a torus with periodicity $2 \pi \kappa, \mathbb{R}_{+}=\{y \in \mathbb{R} \mid y>0\}$, while $v^{\nu}=\left(v_{1}^{\nu}, v_{2}^{\nu}\right)$ and $q^{\nu}$ are respectively the unknown velocity field and pressure field of the fluid. The positive constant $\nu$ is the viscosity coefficient. The vector field $g^{\nu}$ is an external force, decaying fast enough at infinity. The usual no-slip condition is prescribed at $y=0$.

Understanding the behaviour of $v^{\nu}$ for small $\nu$ is a classical and difficult problem: $\nabla v^{\nu}$ tends to blow-up near the boundary as $\nu \rightarrow 0$, and the dynamics of this so-called boundary layer is uneasy to analyze. A main step forward was made by L. Prandtl in 1904, who suggested asymptotics of the form

$$
\begin{aligned}
& v^{\nu}(x, y) \sim\left(V_{1}(x, y / \sqrt{\nu}), \sqrt{\nu} V_{2}(x, y / \sqrt{\nu})\right) \text { near the boundary, } \\
& v^{\nu}(x, y) \sim v^{0}(x, y) \text { away from the boundary, }
\end{aligned}
$$

where $V=\left(V_{1}, V_{2}\right)(x, Y)$ depends on a rescaled variable $Y=y / \sqrt{\nu}$. Hence, in the Prandtl model, the boundary layer has a characteristic scale $\sqrt{\nu}$. Moreover, it connects to an Euler solution $v^{0}$ as $Y \rightarrow+\infty$. By plugging the expansion in (1.1), one obtains a kind of reduced

${ }^{*}$ Université Paris Diderot and IUF, Sorbonne Paris Cité, Institut de Mathématiques de Jussieu-Paris Rive Gauche, UMR 7586, F- 75205 Paris, France

${ }^{\dagger}$ Department of Mathematics, Graduate School of Science, Kyoto University, Kitashirakawa Oiwake-cho, Sakyo-ku, Kyoto 606-8502, Japan 
Navier-Stokes system on $V$, the Prandtl equation, now classical in fluid dynamics. Nevertheless, as pointed out by Prandtl himself, this formal asymptotics is expected to have a limited range of validity, due to an instability phenomenon called boundary layer separation. This instability is typical of flows around obstacles. Roughly, under an adverse pressure gradient in the boundary layer, past a certain distance $x=x_{*}$ from the leading edge of the obstacle, the stress $\left.\partial_{y} v_{1}^{\nu}\right|_{y=0}$ may vanish. This leads to the appearance of a reverse flow for $x>x_{*}$, and detachment of the boundary layer streamlines: see [35, page 39] for a more detailed description of the underlying physics and illustrations.

Mathematically, the importance of this phenomenon has been well recognized in the analysis of the steady Prandtl model. On one hand, it is known from the works of Oleinik [32] that given a horizontal velocity $V_{1}$ at $x=0$ satisfying $\left.V_{1}\right|_{x=0}>0,\left.\partial_{Y} V_{1}\right|_{x=0, Y=0}>0$, one can construct a local in $x$ smooth solution of the Prandtl equation. This result is based on the socalled Von Mises transform, which turns the Prandtl equation into a nonlinear heat equation, with $x$ as an evolution variable. Moreover, this smooth solution exists as long as $V_{1}>0$ and $\left.\partial_{Y} V_{1}\right|_{Y=0}>0$. On the other hand, there exists blowing-up solutions: it was established recently in [4], see also $[14,30,6]$. Still, these results leave aside the behaviour of the full system (1.1), and the justification of the Prandtl asymptotics (1.2) prior to separation. The purpose of the present paper is to contribute to fill in this gap.

Let us stress that most recent mathematical results on the validity of the Prandtl asymptotics are actually related to the unsteady Navier-Stokes equations. In such case, it is now wellunderstood that the justification of the Prandtl approach requires stringent assumptions on the data. The underlying reason is the presence of many hydrodynamic instabilities. We refer for instance to $[3,20,9]$ for discussions and numerics around the various blow-up scenarios. Even to hope for short time stability, one must impose either restrictions on the structure of the perturbations $[27,31]$, or strong regularity assumptions. As regards the well-posedness of the Prandtl model, we refer to [26, 22, 10, 2, 29, 37, 12, 23] and citations therein. As regards the full Navier-Stokes model, a complete justification of the Prandtl theory was obtained for analytic data $[33,34,36]$ and for the initial vorticity supported away from the boundary $[28,7]$. On the contrary, counterexamples to the $H^{1}$ stability of Prandtl expansions of shear flow type was provided by Grenier in [15], using boundary layer profiles with inflexion points. Even in the favourable case of monotonic and concave boundary layer profiles, the boundary layer expansion (1.2) is not stable in a Sobolev framework. This is due to a viscous instability mechanism, the so-called Tollmien-Schlichting wave. This instability, identified in the first half of the 20th century [5], was examined in a nice article by Grenier, Guo and Nguyen [16]. Properly rescaled, their analysis provides highly growing eigenmodes of the linearized Navier-Stokes system around a shear flow of Prandtl type. These eigenmodes have high $x$ frequency $n \sim \nu^{-3 / 8}$, and associated growth rate $\sigma \sim n^{2 / 3} \sim \nu^{-1 / 4}$. For arbitrary small $\nu$, these high frequencies must have very small initial amplitude to be controlled on a time scale independent of $\nu$ : namely, one can only hope for a short time stability result in functional spaces of Gevrey class $3 / 2$ in $x$. A result in this direction was obtained recently by the authors and N. Masmoudi in [11]. See [17] for related statements.

In view of the complexity of the unsteady framework, one could think that justifying Prandtl expansions in the steady case should require stringent assumptions. Surprisingly, we will be able to show local in space Sobolev stability of shear flow solutions of Prandtl type: $U^{\nu}(x, y)=\left(U_{s}(y / \sqrt{\nu}), 0\right)$, under the main assumptions $U_{s}(Y)>0$ for $Y>0, U_{s}(0)=0$, and $U_{s}^{\prime}(0)>0$. These assumptions are somehow minimal in view of the previous discussion: they forbid reverse flow and boundary layer separation. As far as we know, this is the first boundary layer stability result for the steady Navier-Stokes equations with the usual no- 
slip conditions ${ }^{1}$. The only previous articles that we are aware of are [19, 21], dedicated to inhomogeneous Dirichlet conditions. For instance, Guo and Nguyen consider in [19] the steady Navier-Stokes equations in a half-plane, but with a positive Dirichlet datum for the horizontal velocity. They construct general boundary layer expansions for this problem and prove their Sobolev stability through the use of original energy functionals. Let us mention that similar ideas are encountered in the context of the non-stationary MHD equations, where Sobolev stability can be recovered if the magnetic field has a non-vanishing tangential component at the boundary: see $[13,24,25]$. In the present paper, the analysis is of a different nature, and centered on handling the degeneracy due to the homogeneous Dirichlet condition.

We now state precisely our main result. Let $U_{s}=U_{s}(Y) \in C^{2}\left(\overline{\mathbb{R}_{+}}\right)$such that

$$
\begin{aligned}
& U_{s}(0)=0, \quad U_{s}>0 \text { in } Y>0, \quad \lim _{Y \rightarrow \infty} U_{s}(Y)=U_{E}>0, \\
& \partial_{Y} U_{s}(0)>0, \\
& \sum_{k=1,2} \sup _{Y \geq 0}(1+Y)^{3}\left|\partial_{Y}^{k} U_{s}(Y)\right|<\infty .
\end{aligned}
$$

From the continuity and (1.4) we have $\partial_{Y} U_{s}>0$ on $0 \leq Y \leq 4 Y_{0}$ for some $Y_{0} \in(0,1]$. This nondegeneracy near the boundary will be crucial. We then consider the shear flow

$$
U^{\nu}=\left(U_{s}^{\nu}(y), 0\right), \quad U_{s}^{\nu}(y)=U_{s}(y / \sqrt{\nu}) .
$$

Obviously, (1.6) can be seen as a solution of (1.1), setting $g^{\nu}=-\nu \partial_{y}^{2} U^{\nu}$ and $q^{\nu}=0$. The goal of the paper is to establish stability estimates for this solution of boundary layer type. Denoting $u^{\nu}=v^{\nu}-U^{\nu}$ the perturbation induced by $f^{\nu}=g^{\nu}+\nu \partial_{y}^{2} U^{\nu}$, we get

$$
\left\{\begin{array}{l}
U_{s}^{\nu} \partial_{x} u^{\nu}+u_{2}^{\nu} \partial_{y} U_{s}^{\nu} \mathbf{e}_{1}-\nu \Delta u^{\nu}+\nabla p^{\nu}=-u^{\nu} \cdot \nabla u^{\nu}+f^{\nu}, \quad(x, y) \in \mathbb{T}_{\kappa} \times \mathbb{R}_{+}, \\
\operatorname{div} u^{\nu}=0, \quad(x, y) \in \mathbb{T}_{\kappa} \times \mathbb{R}_{+}, \\
\left.u^{\nu}\right|_{y=0}=0 .
\end{array}\right.
$$

Here $\mathbf{e}_{1}=(1,0)$. We then have to specify a functional setting, with $2 \pi \kappa$ periodicity in $x$. Let $\mathcal{P}_{n}, n \in \mathbb{Z}$, be the orthogonal projection on the $n$-th Fourier mode in variable $x$ :

$$
\left(\mathcal{P}_{n} u\right)(x, y)=u_{n}(y) e^{i \tilde{n} x}, \quad \tilde{n}=\frac{n}{\kappa}, \quad u_{n}(y)=\frac{1}{2 \pi \kappa} \int_{0}^{2 \pi \kappa} u(x, y) e^{-i \tilde{n} x} d x
$$

The divergence-free and homogeneous Dirichlet conditions imply $u_{0}=\left(u_{0,1}, 0\right)$. Setting

$$
\mathcal{Q}_{0} u=\left(I-\mathcal{P}_{0}\right) u,
$$

where $I$ is the identity operator, we can identify $u$ with the couple $\left(u_{0,1}, \mathcal{Q}_{0} u\right)$. With this identification we introduce

$$
\begin{array}{r}
X=\left\{\left(u_{0,1}, \mathcal{Q}_{0} u\right) \in B C\left(\overline{\mathbb{R}_{+}}\right) \times W_{0}^{1,2}\left(\mathbb{T}_{\kappa} \times \mathbb{R}_{+}\right)^{2}\left|\quad \partial_{y} u_{0,1} \in L^{2}\left(\mathbb{R}_{+}\right), \quad u_{0,1}\right|_{y=0}=0,\right. \\
\left.\|u\|_{X}=\left\|u_{0,1}\right\|_{L^{\infty}\left(\mathbb{R}_{+}\right)}+\left\|\partial_{y} u_{0,1}\right\|_{L^{2}\left(\mathbb{R}_{+}\right)}+\sum_{n \neq 0}\left\|u_{n}\right\|_{L^{\infty}\left(\mathbb{R}_{+}\right)}+\left\|\mathcal{Q}_{0} u\right\|_{W^{1,2}\left(\mathbb{T}_{\kappa} \times \mathbb{R}_{+}\right)}<\infty\right\},
\end{array}
$$

where the Sobolev space $W_{0}^{1,2}\left(\mathbb{T}_{\kappa} \times \mathbb{R}_{+}\right)$is defined as the subspace of $W^{1,2}\left(\mathbb{T}_{\kappa} \times \mathbb{R}_{+}\right)$with functions having the zero boundary trace on $y=0$. For simplicity we assume that $f^{\nu}=\mathcal{Q}_{0} f^{\nu}$ below, though it is not difficult to extend our result to a general case by imposing a suitable condition on $f_{0}^{\nu}(y)$.

\footnotetext{
${ }^{1}$ see Remark 1.5 for an update.
} 
Theorem 1.1. There exist positive numbers $\kappa_{0}, \nu_{0}, \epsilon$ such that the following statement holds for $0<\kappa \leq \kappa_{0}$ and $0<\nu \leq \nu_{0}$. If $f^{\nu}=\mathcal{Q}_{0} f^{\nu}$ and $\left\|f^{\nu}\right\|_{L^{2}} \leq \epsilon \nu^{\frac{3}{4}}|\log \nu|^{-1}$ then there exists a unique solution $\left(u^{\nu}, \nabla p^{\nu}\right) \in\left(X \cap W_{\text {loc }}^{2,2}\left(\mathbb{T}_{\kappa} \times \mathbb{R}_{+}\right)^{2}\right) \times L^{2}\left(\mathbb{T}_{\kappa} \times \mathbb{R}_{+}\right)^{2}$ to (1.7) such that

$$
\begin{aligned}
& \left\|u_{0,1}^{\nu}\right\|_{L^{\infty}}+\nu^{\frac{1}{4}}\left\|\partial_{y} u_{0,1}^{\nu}\right\|_{L^{2}} \\
& \quad+\sum_{n \neq 0}\left\|u_{n}^{\nu}\right\|_{L^{\infty}}+\nu^{-\frac{1}{4}}\left\|\mathcal{Q}_{0} u^{\nu}\right\|_{L^{2}}+\nu^{\frac{1}{4}}\left\|\nabla \mathcal{Q}_{0} u^{\nu}\right\|_{L^{2}} \leq \frac{C|\log \nu|^{\frac{1}{2}}}{\nu^{\frac{1}{4}}}\left\|f^{\nu}\right\|_{L^{2}},
\end{aligned}
$$

Here $C$ is independent of $\nu$ and $\kappa$.

Remark 1.2. The main structural assumptions of our stability theorems are (1.3) and (1.4), which are natural in view of the previous comments on boundary layer separation. Another important requirement is the smallness condition on $\kappa$ : it means that our stability result is only local in space (although on a lengthscale independent of $\nu$ ). An interesting question is wether this locality requirement is only a technical restriction of our stability method, or if instabilities are possible at larger wavelengths.

Remark 1.3. The perturbation $u^{\nu}$ converges (at least in a weak sense) to a constant shear flow at infinity:

$$
\lim _{y \rightarrow+\infty} v^{\nu}=\left(c^{\nu}, 0\right)
$$

First, the requirement $\mathcal{Q}_{0} u^{\nu} \in W^{1,2}$ implies that $\mathcal{Q}_{0} u^{\nu}$ goes to zero at infinity. Then, as regards the $x$-average $u_{0}^{\nu}=\left(u_{0,1}^{\nu}, 0\right)$, we deduce from the first line of (1.7) and the fact that $f_{0}^{\nu}=0$ :

$$
-\nu \partial_{y}^{2} u_{0,1}^{\nu}=-\partial_{y}\left(Q_{0} u_{2}^{\nu} Q_{0} u_{1}^{\nu}\right)_{0} .
$$

As $\partial_{y} u_{0,1} \in L^{2}$, we can integrate this identity from $y=+\infty$ to deduce

$$
-\nu \partial_{y} u_{0,1}^{\nu}=-\left(Q_{0} u_{2}^{\nu} Q_{0} u_{1}^{\nu}\right)_{0}
$$

Eventually, as the right-hand side belongs to $L^{1}$, we find (1.12) with

$$
c^{\nu}=\frac{1}{\nu} \int_{\mathbb{R}_{+}}\left(Q_{0} u_{2}^{\nu} Q_{0} u_{1}^{\nu}\right)_{0} .
$$

Note that this constant at infinity can not be prescribed. Moreover, it obeys the bound

$$
\left|c^{\nu}\right| \leq \frac{C|\log \nu|^{\frac{1}{2}}}{\nu^{\frac{1}{4}}}\left\|f^{\nu}\right\|_{L^{2}}
$$

as a consequence of estimate (1.11).

Remark 1.4. Theorem 1.1 has an easy implication on the inviscid limit problem. Namely, any solution $v^{\nu}$ of (1.1) associated to a source term of the form $g^{\nu}=-\nu \partial_{y}^{2} U^{\nu}+f^{\nu}$, where $U^{\nu}$ satisfies (1.3)-(1.4)-(1.5)-(1.6) and $\left\|f^{\nu}\right\|_{L^{2}}=o\left(\nu^{3 / 4} /|\log \nu|^{-1}\right)$, converges to the Euler solution $v^{0}=\left(U_{E}, 0\right)$ in $L_{l o c}^{\infty} \cap H_{l o c}^{1}$. This is the first non-trivial example of a class of steady Navier-Stokes solutions for which the inviscid limit holds true in finite regularity.

Remark 1.5. (Update 07/31/2018) Just after our manuscript submission on the arXiv, Y. Guo and S. Iyer have submitted the very interesting preprint [18]. They establish there the Sobolev stability of a subclass of Prandtl expansions, the main example of which being the famous Blasius flow. We feel that this work and ours are complementary: the focus of [18] is the very important Blasius self-similar solution (with $x$-dependence), while our analysis is 
more elementary: it treats the simpler case of shear flows under minimal assumptions. In this perspective, we hope that our paper will be a first step in the stability analysis of general steady boundary layer expansions, able to cover both works ${ }^{2}$. Moreover, beyond the context of boundary layer flows, we feel that our analysis may provide new tools to investigate the stability of shear flows, which is a classical topic in hydrodynamics.

To conclude this introduction, we give the outline of the proof of Theorem 1.1. After collecting a few useful estimates in Section 2, we will turn to the core of the proof, which is the analysis of the linearized system around $U^{\nu}$. Through a Fourier transform in $x$, it can be written

$$
\left\{\begin{array}{l}
i \tilde{n} U_{s}^{\nu} u_{n}+u_{n, 2}\left(\partial_{y} U_{s}^{\nu}\right) \mathbf{e}_{1}-\nu\left(\partial_{y}^{2}-\tilde{n}^{2}\right) u_{n}+\left(\begin{array}{c}
i \tilde{n} p_{n} \\
\partial_{y} p_{n}
\end{array}\right)=f_{n}, \quad y>0, \\
i \tilde{n} u_{n, 1}+\partial_{y} u_{n, 2}=0, \quad y>0 \\
\left.u_{n}\right|_{y=0}=0
\end{array}\right.
$$

We remind that $u_{n}=u_{n}(y)$ is the $n$-th Fourier coefficient of the velocity, and $\tilde{n}=n / \kappa$. Note that $| \pm \tilde{1}|$ is large if $\kappa$ is small. The zero mode does not raise any difficulty, and is estimated in Section 3. The difficult part is the derivation of good bounds for $\tilde{n} \neq 0$, see Theorem 4.1. For $\kappa$ small enough, we can always ensure that $|\tilde{n}| \gg 1$ for all $n$. Nevertheless, as $\nu \ll 1$, the tangential diffusion term $-\nu \tilde{n}^{2} u_{n}$ in the first line of (1.13) is in general far too small to control the stretching term $u_{n, 2} \partial_{y} U_{s}^{\nu}=O\left(\frac{1}{\sqrt{\nu}}\left|u_{n}\right|\right)$.

To obtain good bounds, we distinguish between two regimes: $|\tilde{n}| \ll \nu^{-3 / 4}$ and $|\tilde{n}| \gtrsim \nu^{-3 / 4}$. The regime $|\tilde{n}| \gtrsim \nu^{-3 / 4}$ is handled in Section 8, through a direct analysis of system (1.13). The subcase $|\tilde{n}| \gg \nu^{-3 / 4}$ can be treated by simple energy estimates, $c f$ item i) in Proposition 8.1: the diffusion term is enough to control stretching by the boundary layer velocity. However, the regime where $|\tilde{n}| \sim \nu^{-3 / 4}$ is much more difficult, and requires new estimates. Such estimates, in which the convection term is involved, are actually valid in the wider regime $\nu^{-1 / 2} \ll|\tilde{n}| \lesssim \nu^{-3 / 4}$, cf item ii) in Proposition 8.1.

Stability in the regime $|\tilde{n}| \ll \nu^{-3 / 4}$ is the most delicate to obtain. It is deduced from a careful analysis of the steady Orr-Sommerfeld system (4.12), which is a reformulation of (1.13) in terms of the stream function and of the rescaled variable $Y=y / \sqrt{\nu}$. It reads

$$
\left\{\begin{array}{l}
O S[\phi]:=U_{s}\left(\partial_{Y}^{2}-\alpha^{2}\right) \phi-U_{s}^{\prime \prime} \phi+i \varepsilon\left(\partial_{Y}^{2}-\alpha^{2}\right)^{2} \phi=-f_{2}-\frac{i}{\alpha} \partial_{Y} f_{1}, \quad Y>0, \\
\left.\phi\right|_{Y=0}=\left.\partial_{Y} \phi\right|_{Y=0}=0 .
\end{array}\right.
$$

where parameters $\alpha$ and $\varepsilon$ are related to the tangential frequency $\tilde{n}$ and the viscosity $\nu$. In short, the regime $|\tilde{n}| \ll \nu^{-3 / 4}$ corresponds to the case $\varepsilon^{1 / 3} \alpha \ll 1$.

The point is that we are not able to get direct estimates on this system. Instead, we construct the solution through an iterative process, reminiscent of splitting methods in numerical analysis. More precisely, one main idea is to construct a solution to the Orr-Sommerfeld equation in the form of a series, where successive corrections solve alternatively:

- inviscid approximations of the equation, based on the so-called Rayleigh equation.

- viscous approximations of the equation, based on the so-called Airy equation.

This idea of a splitting method was already present in our Gevrey stability study of the unsteady case [11], and found its origin in article [16]: the construction of an unstable eigenmode for the linearized Navier-Stokes equations was performed with a similar iteration, although more explicit and specific to a narrower regime of parameters. Here and in [11], the convergence of the iteration is rather shown by energy arguments, and adapted to the whole

\footnotetext{
${ }^{2}$ As regards the unsteady case, extension of article [11] to general $x$-dependent expansions is in progress.
} 
range $|\tilde{n}| \ll \nu^{-3 / 4}$. But in the steady setting considered here, we must rely on estimates that are totally different from the ones in [11], in order to reach Sobolev stability. Moreover, the implementation of the splitting method is different.

The inviscid estimates are established in Section 5: they are mostly about the equation $\operatorname{Ray}[\varphi]=f$, where the Rayleigh operator Ray $:=U_{s}\left(\partial_{Y}^{2}-\alpha^{2}\right)-U_{s}^{\prime \prime}$ corresponds to neglecting the diffusion in the Orr-Sommerfeld operator. Due to the degeneracy of $U_{s}$ at $Y=0$, the derivation of good bounds is uneasy, and provided in Proposition 5.1. The most difficult case is when $\alpha \ll 1$ : indeed taking $\alpha \rightarrow 0$ in the Rayleigh equation yields a singular perturbation problem, $c f$ Remark 5.2. Nevertheless, as shown in the estimates of Proposition 5.1, a crucial point is that the singularity shows up only when the source term $f$ has nonzero average in $Y$.

After the inviscid analysis of Section 5, Section 6 collects various estimates on viscous equations of Airy type: they all involve the operator Airy $:=U_{s}+i \varepsilon\left(\partial_{Y}^{2}-\alpha^{2}\right)$. Note that the Rayleigh and Airy operators are naturally involved within the full Orr-Sommerfeld operator through the identities (to be detailed later):

$$
\begin{aligned}
& O S[\phi]=\operatorname{Ray}[\phi]+i \varepsilon\left(\partial_{Y}^{2}-\alpha^{2}\right)^{2} \phi=\operatorname{Ray}[\phi]+i \varepsilon\left(\partial_{Y}^{2}-\alpha^{2}\right)\left[\frac{1}{U_{s}} \operatorname{Ray}[\phi]+\frac{U_{s}^{\prime \prime}}{U_{s}} \phi\right], \\
& O S[\phi]=\left(\partial_{Y}^{2}-\alpha^{2}\right) \operatorname{Airy}[\phi]-2 \partial_{Y}\left(U_{s}^{\prime} \phi\right), \\
& O S[\phi]=\operatorname{Airy}\left[\frac{1}{U_{s}} \operatorname{Ray}[\phi]\right]+i \varepsilon\left(\partial_{Y}^{2}-\alpha^{2}\right) \frac{U_{s}^{\prime \prime}}{U_{s}} \phi
\end{aligned}
$$

These identities are at the basis of the splitting method alluded to above, which provides a solution to the Orr-Sommerfeld equation under the form of a converging series. This construction, called Rayleigh-Airy iteration, is described in Subsection 7.1. In this process, a special attention is paid to the possible singularity generated by the Rayleigh equation when $\alpha \ll 1$, which could forbid the convergence of the series. In short, one has to ensure that each "Rayleigh step" is performed with a zero average source term. This major difficulty is new compared to the unsteady analysis in [11], and leads to a different iteration.

Moreover, the Rayleigh-Airy iteration is not enough to conclude: it provides a solution to the Orr-Sommerfeld equation with a given source term, but this solution does not satisfy both Dirichlet and Neumann conditions. Only the Dirichlet condition is maintained through the iteration. One must then combine it with two solutions of the homogeneous Orr-Sommerfeld equation (with an inhomogeneous Dirichlet condition $\left.\phi\right|_{Y=0}=1$ ). These special solutions $\phi_{\text {slow }}$ and $\phi_{\text {fast }}$ are called slow and fast modes, following a terminology of [16]. They are built in Subsection 7.3 (see also the preliminary results given in Corollay 5.5 and Proposition 5.6) and Subsection 7.4 respectively. Let us stress that the construction of the slow and fast modes can not be performed in an abstract way, like for the solution coming from the Rayleigh-Airy iteration. They are rather obtained starting from an explicit approximation (of inviscid type for the slow mode, of viscous "boundary layer type" for the fast mode), which fulfills the inhomogeneous condition, but solves approximately the equation. One can then add a corrector to get an exact solution, notably making use of the Rayleigh-Airy iteration developped earlier. The proof of the linear stability result in the regime $|\tilde{n}| \ll \nu^{-3 / 4}$ is then achieved in Paragraphs 7.5 and 7.6.

Once the linear estimates of Theorem 4.1 are shown, the proof of our main Theorem 1.1 can be completed classically by a fixed point argument. This is done in Section 9 .

\section{Preliminaries}

We collect here a few estimates to appear in the next sections. Assume that $U_{s}$ satisfies (1.3)-(1.5), and let $Y_{0} \in(0,1]$ be a number such that $\partial_{Y} U_{s}>0$ holds on $0 \leq Y \leq 4 Y_{0}$. 
Proposition 2.1. (1) The following inequalities hold:

$$
\begin{aligned}
& \left\|\frac{Y \partial_{Y} U_{s}}{U_{s}}\right\|_{L_{Y}^{2}}+\left\|\frac{Y \partial_{Y}^{2} U_{s}}{U_{s}}\right\|_{L_{Y}^{\infty}}<\infty, \\
& \left|\partial_{y}^{2} U_{s}^{\nu}(y)\right| \leq \frac{C}{\nu^{\frac{1}{2}}} \partial_{y} U_{s}^{\nu}(y), \quad 0 \leq y \leq 2 Y_{0} \nu^{\frac{1}{2}} .
\end{aligned}
$$

(2) $\operatorname{Set} G_{s}(Y)=U_{s}(Y) \int_{Y}^{Y_{0}} \frac{1}{U_{s}^{2}} d Y_{1}$. Then

$$
\partial_{Y}\left(U_{s}^{2} \partial_{Y}\left(\frac{G_{s}}{U_{s}}\right)\right)=0 \quad \text { for } \quad Y>0, \quad G_{s}(0)=\frac{1}{\partial_{Y} U_{s}(0)},
$$

and

$$
\left\|\frac{G_{s}}{1+Y}\right\|_{L_{Y}^{\infty}}+\left\|\frac{\partial_{Y} G_{s}}{\log Y}\right\|_{L_{Y}^{\infty}(\{0<Y \leq 1 / 2\})}+\left\|\partial_{Y} G_{s}\right\|_{L_{Y}^{\infty}(\{Y \geq 1 / 2\})}<\infty .
$$

Proof. The results of (1) are straightforward, so we only give the proof of (2). It is easy to see that $G_{s}$ satisfies the equation as in the claim. Moreover, we see

$$
G_{s}(Y)=-U_{s}(Y) \int_{Y}^{Y_{0}}\left(\frac{1}{U_{s}}\right)^{\prime} \frac{d Y_{1}}{U_{s}^{\prime}}=\frac{1}{U_{s}^{\prime}(Y)}-\frac{U_{s}(Y)}{U_{s}\left(Y_{0}\right) U_{s}^{\prime}\left(Y_{0}\right)}-U_{s}(Y) \int_{Y}^{Y_{0}} \frac{1}{U_{s}} \frac{U_{s}^{\prime \prime}}{\left(U_{s}^{\prime}\right)^{2}} d Y_{1} .
$$

Thus, we have $G_{s}(0)=\frac{1}{U_{s}^{\prime}(0)}$, and we also have

$$
\partial_{Y} G_{s}(Y)=-\frac{U_{s}^{\prime}(Y)}{U_{s}\left(Y_{0}\right) U_{s}^{\prime}\left(Y_{0}\right)}-U_{s}^{\prime}(Y) \int_{Y}^{Y_{0}} \frac{1}{U_{s}} \frac{U_{s}^{\prime \prime}}{\left(U_{s}^{\prime}\right)^{2}} d Y_{1}
$$

Thus, the fact $U_{s} \sim U_{s}^{\prime}(0) Y$ for $0<Y \ll 1$ implies $\left\|\frac{\partial_{Y} G_{s}}{\log Y}\right\|_{L_{Y}^{\infty}(\{0<Y<1 / 2\})}<\infty$. On the other hand, since $U_{s} \sim U_{E}$ for $Y \gg 1$ and $U_{s}>0$ in $Y>0$, it is easy to show that $\left\|\frac{G_{s}}{1+Y}\right\|_{L_{Y}^{\infty}}+\left\|\partial_{Y} G_{s}\right\|_{L_{Y}^{\infty}(\{Y \geq 1 / 2\})}<\infty$. The proof is complete.

Proposition 2.2. (1) Let $\sigma[\cdot]$ be the linear operator defined by

$$
\sigma[f](Y)=\int_{Y}^{\infty} f d Y_{1}, \quad f \in C_{0}^{\infty}\left(\overline{\mathbb{R}_{+}}\right) .
$$

Then for $1 \leq p<\infty$ and $k=0,1, \ldots$,

$$
\left\|Y^{k} \sigma[f]\right\|_{L_{Y}^{p}} \leq C_{p}\left\|Y^{k+1} f\right\|_{L_{Y}^{p}} .
$$

(2) Let $L[\cdot]$ be the linear operator defined by

$$
L[f](Y)=U_{s}(Y) \int_{Y}^{\infty} \frac{f}{U_{s}^{2}} d Y_{1}, \quad f \in C_{0}^{\infty}\left(\overline{\mathbb{R}_{+}}\right) .
$$

Then for $1<p<\infty$ and $k=0,1, \ldots$,

$$
\begin{aligned}
\left\|Y^{k} L[f]\right\|_{L_{Y}^{p}} & \leq C\left\|Y^{k}(1+Y) f\right\|_{L_{Y}^{p}}, \\
\left\|\partial_{Y} L[f]\right\|_{L_{Y}^{2}} & \leq C\left(\|f\|_{L_{Y}^{1}}+\|f\|_{L_{Y}^{2}}+\left\|\partial_{Y} f\right\|_{L_{Y}^{2}}\right) .
\end{aligned}
$$

Remark 2.3. In the estimate of $\partial_{Y} L[f]$ we do not need the condition $\left.f\right|_{Y=0}=0$. Thus, the estimate is valid for any $f \in L^{1}\left(\mathbb{R}_{+}\right) \cap H^{1}\left(\mathbb{R}_{+}\right)$. 
Proof. (1) Since $Y^{k}|\sigma[f](Y)| \leq \sigma\left[\left|f_{k}\right|\right](Y)$ with $f_{k}(Y)=Y^{k} f(Y)$ pointwisely, it suffices to consider the case $k=0$. By changing the order of the integral we see $\|\sigma[f]\|_{L_{Y}^{1}} \leq\|Y f\|_{L_{Y}^{1}}$, while we have for $1<p<\infty,|\sigma[f](Y)| \leq C_{p} Y^{-1 / p}\|Y f\|_{L_{Y}^{p}}$, which implies $\|\sigma[f]\|_{L_{Y}^{p, \infty}} \leq$ $C_{p}\|Y f\|_{L_{Y}^{p}}$. Thus the Marcinkiewicz interpolation theorem gives $\|\sigma[f]\|_{L_{Y}^{p}} \leq C\|Y f\|_{L_{Y}^{p}}$. The proof is complete.

(2) It suffices to consider the case $k=0$. Since $U_{s} \sim U_{s}^{\prime}(0) Y$ for $0<Y \ll 1$ and $U_{s} \sim U_{E}$ for $Y \gg 1$ we observe that

$$
|L[f](Y)| \leq \frac{C_{p}}{Y^{\frac{1}{p}}}\|(1+Y) f\|_{L_{Y}^{p}}, \quad Y>0 .
$$

Thus $\|L[f]\|_{L_{Y}^{p, \infty}} \leq C_{p}\|(1+Y) f\|_{L_{Y}^{p}}$ holds for $1<p<\infty$, and hence, the Marcinkiewicz interpolation theorem yields $\|L[f]\|_{L_{Y}^{p}} \leq C\|(1+Y) f\|_{L_{Y}^{p}}$ for $1<p<\infty$. Next we see

$$
\partial_{Y} L[f](Y)=U_{s}^{\prime}(Y) \int_{Y}^{\infty} \frac{f}{U_{s}^{2}} d Y_{1}-\frac{f}{U_{s}}=U_{s}^{\prime}(Y) \int_{Y}^{Y_{0}} \frac{f}{U_{s}^{2}} d Y_{1}+U_{s}^{\prime}(Y) \int_{Y_{0}}^{\infty} \frac{f}{U_{s}^{2}} d Y_{1}-\frac{f}{U_{s}} .
$$

From this expression it is easy to see that

$$
\left\|\partial_{Y} L[f]\right\|_{L^{2}\left(\left\{Y \geq Y_{0}\right\}\right)} \leq C\left(\|f\|_{L_{Y}^{1}}+\|f\|_{L_{Y}^{2}}\right),
$$

and also

$$
\left\|U_{s}^{\prime}(Y) \int_{Y_{0}}^{\infty} \frac{f}{U_{s}^{2}} d Y_{1}\right\|_{L_{Y}^{2}} \leq C\|f\|_{L_{Y}^{1}}
$$

Now it suffices to consider the estimate of $U_{s}^{\prime}(Y) \int_{Y}^{Y_{0}} \frac{f}{U_{s}^{2}} d Y_{1}-\frac{f}{U_{s}}$ in $0<Y \leq Y_{0}$. We see

$$
\begin{aligned}
U_{s}^{\prime}(Y) \int_{Y}^{Y_{0}} \frac{f}{U_{s}^{2}} d Y_{1}-\frac{f}{U_{s}} & =-U_{s}^{\prime}(Y) \int_{Y}^{Y_{0}}\left(\frac{1}{U_{s}}\right)^{\prime} \frac{f}{U_{s}^{\prime}} d Y_{1}-\frac{f}{U_{s}} \\
& =-\frac{U_{s}^{\prime}(Y) f\left(Y_{0}\right)}{U_{s}\left(Y_{0}\right) U_{s}^{\prime}\left(Y_{0}\right)}+U_{s}^{\prime}(Y) \int_{Y}^{Y_{0}} \frac{1}{U_{s}}\left(\frac{f}{U_{s}^{\prime}}\right)^{\prime} d Y_{1} .
\end{aligned}
$$

It is clear that

$$
\left\|\frac{U_{s}^{\prime}(Y) f\left(Y_{0}\right)}{U_{s}\left(Y_{0}\right) U_{s}^{\prime}\left(Y_{0}\right)}\right\|_{L^{2}\left(\left\{0<Y \leq Y_{0}\right\}\right)} \leq C\left(\left\|\partial_{Y} f\right\|_{L_{Y}^{2}}+\|f\|_{L_{Y}^{2}}\right)
$$

by applying the embedding inequality $\|f\|_{L_{Y}^{\infty}} \leq C\left\|\partial_{Y} f\right\|_{L_{Y}^{2}}^{\frac{1}{2}}\|f\|_{L_{Y}^{2}}^{\frac{1}{2}}$ (note that the condition $\left.f\right|_{Y=0}=0$ is not required). Let us consider the estimate of $\left\|U_{s}^{\prime}(Y) \int_{Y}^{Y_{0}} \frac{1}{U_{s}}\left(\frac{f}{U_{s}^{\prime}}\right)^{\prime} d Y_{1}\right\|_{L^{2}\left(\left\{0<Y \leq Y_{0}\right\}\right)}$. We see

$$
\left|U_{s}^{\prime}(Y) \int_{Y}^{Y_{0}} \frac{1}{U_{s}}\left(\frac{f}{U_{s}^{\prime}}\right)^{\prime} d Y_{1}\right| \leq \frac{C}{Y^{\frac{1}{p}}}\left\|\left(\frac{f}{U_{s}^{\prime}}\right)^{\prime}\right\|_{L^{p}\left(\left\{0<Y \leq Y_{0}\right\}\right)}, \quad 1<p<\infty, \quad 0<Y \leq Y_{0} .
$$

Hence the Marcinkiewicz interpolation theorem yields

$$
\left\|U_{s}^{\prime}(Y) \int_{Y}^{Y_{0}} \frac{1}{U_{s}}\left(\frac{f}{U_{s}^{\prime}}\right)^{\prime} d Y_{1}\right\|_{L^{2}\left(\left\{0<Y \leq Y_{0}\right\}\right)} \leq C\left\|\left(\frac{f}{U_{s}^{\prime}}\right)^{\prime}\right\|_{L^{2}\left(\left\{0<Y \leq Y_{0}\right\}\right)} \leq C\left(\left\|\partial_{Y} f\right\|_{L_{Y}^{2}}+\|f\|_{L_{Y}^{2}}\right) .
$$

The proof is complete.

We end this short section with an interpolation inequality that will be applied several times. 
Proposition 2.4. There exists $C>0$ such that

$$
\|g\|_{L_{Y}^{2}}^{2} \leq C\left\|\sqrt{U_{s}} g\right\|_{L_{Y}^{2}}^{\frac{4}{3}}\left\|\partial_{Y} g\right\|_{L_{Y}^{2}}^{\frac{2}{3}}+C\left\|\sqrt{U_{s}} g\right\|_{L_{Y}^{2}}^{2}, \quad g=g(Y) \in H^{1}\left(\mathbb{R}_{+}\right) .
$$

Proof. This bound is an easy consequence of the inequality

$$
\|g\|_{L_{Y}^{2}}^{2} \leq C\|\sqrt{Y} g\|_{L_{Y}^{2}}\|g\|_{L_{Y}^{\infty}} \leq C\|\sqrt{Y} g\|_{L_{Y}^{2}}\left\|\partial_{Y} g\right\|_{L_{Y}^{2}}^{\frac{1}{2}}\|g\|_{L_{Y}^{2}}^{\frac{1}{2}}
$$

which implies

$$
\|g\|_{L_{Y}^{2}} \leq C\|\sqrt{Y} g\|_{L_{Y}^{2}}^{\frac{2}{3}}\left\|\partial_{Y} g\right\|_{L_{Y}^{2}}^{\frac{1}{3}}
$$

and of the properties of $U_{s}: U_{s}(Y) \sim U_{s}^{\prime}(0) Y$ around $Y=0, U_{s}(Y) \sim U_{E}>0$ around $Y=\infty$, together with the assumption $U_{s}>0$ for any $Y>0$. The first inequality is obtained by writing that for all $A>0$

$$
\|g\|_{L_{Y}^{2}}^{2} \leq \int_{0}^{A}|g|^{2}+\int_{A}^{+\infty}|g|^{2} \leq A\|g\|_{L_{Y}^{\infty}}^{2}+\frac{1}{A}\|\sqrt{Y} g\|_{L_{Y}^{2}}^{2}
$$

and optimizing in $A$. The proof is complete.

Note that an interpolation inequality of this type is also used in the work [8] to construct a steady Navier-Stokes flow around a rotating disk.

\section{Linear result for the zero mode}

When $n=0$ the linearized problem (1.13) is reduced to a simple ODE: indeed, $u_{0,2}=0$ and $\nu \partial_{y}^{2} u_{0,1}=f_{0,1}$ with $\left.u_{0,1}\right|_{y=0}=0$. Then $u_{0,1}(y)=-\frac{1}{\nu} \int_{0}^{y} \int_{y^{\prime}}^{\infty} f_{0,1}\left(y^{\prime \prime}\right) d y^{\prime \prime} d y^{\prime}$. The pressure $p_{0}$ is given by $p_{0}(y)=-\int_{y}^{\infty} f_{0,2}\left(y^{\prime}\right) d y^{\prime}$. Hence we have

Theorem 3.1. Let $f_{0} \in L^{1}\left(\mathbb{R}_{+}^{2}\right)^{2}$ and $f_{0,1}=\partial_{y} F_{0,1}$ with $F_{0,1} \in L^{1}\left(\mathbb{R}_{+}\right) \cap L^{2}\left(\mathbb{R}_{+}\right)$. Then there exists a unique solution $u_{0}=\left(u_{0,1}, 0\right)^{\top}$ to $(1.13)$ with $\tilde{n}=0$ such that

$$
\begin{aligned}
\left\|u_{0,1}\right\|_{L^{\infty}} & \leq \frac{1}{\nu}\left\|F_{0,1}\right\|_{L^{1}}, \\
\left\|\partial_{y} u_{0,1}\right\|_{L^{2}} & =\frac{1}{\nu}\left\|F_{0,1}\right\|_{L^{2}} .
\end{aligned}
$$

We also have $\lim _{y \rightarrow \infty} u_{0,1}=\frac{1}{\nu} \int_{0}^{\infty} F_{0,1} d y$.

\section{Linear result for the non-zero modes}

In this section we state the main result for the linearized problem (1.13) when $\tilde{n} \neq 0$.

Theorem 4.1. There exist positive numbers $\kappa_{0}, \nu_{0}$, and $\delta_{*}$ such that the following statement holds for any $0<\kappa \leq \kappa_{0}, 0<\nu \leq \nu_{0}$, and $\tilde{n} \neq 0$. For any $f_{n} \in L^{2}\left(\mathbb{R}_{+}\right)^{2}$ there exists a unique solution $u_{n} \in H^{2}\left(\mathbb{R}_{+}\right)^{2} \cap H_{0}^{1}\left(\mathbb{R}_{+}\right)^{2}$ to (1.13) satisfying the estimates stated below: 
(i) if $0<|\tilde{n}| \leq \nu^{-\frac{3}{7}}$ then

$$
\begin{aligned}
\left\|u_{n}\right\|_{L^{2}} & \leq \begin{cases}\frac{C}{|\tilde{n}|^{\frac{1}{2}}}\left\|f_{n}\right\|_{L^{2}}, & 0<|\tilde{n}| \leq \nu^{-\frac{3}{8}} \\
\frac{C}{|\tilde{n}|^{\frac{11}{6}} \nu^{\frac{1}{2}}}\left\|f_{n}\right\|_{L^{2}}, & \nu^{-\frac{3}{8}} \leq|\tilde{n}| \leq \nu^{-\frac{3}{7}}\end{cases} \\
\left\|u_{n}\right\|_{L^{\infty}} & \leq \frac{C}{|\tilde{n}|^{\frac{1}{2}} \nu^{\frac{1}{4}}}\left\|f_{n}\right\|_{L^{2}}, \\
\left\|\partial_{y} u_{n}\right\|_{L^{2}}+|\tilde{n}|\left\|u_{n}\right\|_{L^{2}} & \leq \frac{C}{|\tilde{n}|^{\frac{1}{3}} \nu^{\frac{1}{2}}}\left\|f_{n}\right\|_{L^{2}} .
\end{aligned}
$$

(ii) if $\nu^{-\frac{3}{7}} \leq|\tilde{n}| \leq \delta_{*} \nu^{-\frac{3}{4}}$ then

$$
\begin{aligned}
\left\|u_{n}\right\|_{L^{2}} & \leq \frac{C}{|\tilde{n}|^{\frac{2}{3}}}\left\|f_{n}\right\|_{L^{2}}, \\
\left\|\partial_{y} u_{n}\right\|_{L^{2}}+|\tilde{n}|\left\|u_{n}\right\|_{L^{2}} & \leq \frac{C}{|\tilde{n}|^{\frac{1}{3}} \nu^{\frac{1}{2}}}\left\|f_{n}\right\|_{L^{2}} .
\end{aligned}
$$

(iii) if $|\tilde{n}| \geq \delta_{*} \nu^{-\frac{3}{4}}$ then

$$
\begin{aligned}
\left\|u_{n}\right\|_{L^{2}} & \leq \frac{C}{|\tilde{n}|^{2} \nu}\left\|f_{n}\right\|_{L^{2}}, \\
\left\|\partial_{y} u_{n}\right\|_{L^{2}}+|\tilde{n}|\left\|u_{n}\right\|_{L^{2}} & \leq \frac{C}{|\tilde{n}| \nu}\left\|f_{n}\right\|_{L^{2}} .
\end{aligned}
$$

Remark 4.2. (1) In Theorem 4.1, the associated pressure $p_{n}$ belongs to $H^{1}\left(\mathbb{R}_{+}\right)$.

(2) Estimate (4.2) is not a consequence of the interpolation between (4.1) and (4.3). On the other hand, by the interpolation inequality $\left\|u_{n}\right\|_{L^{\infty}} \leq C\left\|\partial_{y} u_{n}\right\|_{L^{2}}^{\frac{1}{2}}\left\|u_{n}\right\|_{L^{2}}^{\frac{1}{2}}$, we have from (4.4), (4.5), (4.6), and (4.7),

$$
\left\|u_{n}\right\|_{L^{\infty}} \leq \begin{cases}\frac{C}{|\tilde{n}|^{\frac{1}{2}} \nu^{\frac{1}{4}}}\left\|f_{n}\right\|_{L^{2}}, & \nu^{-\frac{3}{7}} \leq|\tilde{n}| \leq \delta_{*} \nu^{-\frac{3}{4}}, \\ \frac{C}{|\tilde{n}|^{\frac{3}{2}} \nu}\left\|f_{n}\right\|_{L^{2}}, & |\tilde{n}| \geq \delta_{*} \nu^{-\frac{3}{4}}\end{cases}
$$

The proof of Theorem 4.1 consists of several steps, and is given in Sections 5 - 8 below. The core part of the proof is the study of the Orr-Sommerfeld equation for the streamfunction. For the moment we assume that $f_{n}$ is smooth enough, say, $f_{n} \in H^{1}\left(\mathbb{R}_{+}\right)^{2}$. The Orr-Sommerfeld equation is deduced from the equation for the vorticity $\omega_{n}=i \tilde{n} u_{n, 2}-\partial_{y} u_{n, 1}$ :

$$
i \tilde{n} U_{s}^{\nu} \omega_{n}-u_{n, 2} \partial_{y}^{2} U_{s}^{\nu}-\nu\left(\partial_{y}^{2}-\tilde{n}^{2}\right) \omega_{n}=i \tilde{n} f_{n, 2}-\partial_{y} f_{n, 1}, \quad y>0 .
$$

Let us introduce the streamfunction $\phi_{n}$ as the solution to the Poisson equation

$$
-\left(\partial_{y}^{2}-\tilde{n}^{2}\right) \phi_{n}=\omega_{n},\left.\quad \phi_{n}\right|_{y=0}=0 .
$$

The $n$th mode of the velocity $u_{n}$ is recovered from the formula

$$
u_{n, 1}=\partial_{y} \phi_{n}, \quad u_{n, 2}=-i \tilde{n} \phi_{n} .
$$

Taking into account no-slip boundary condition on $u_{n, 1}$, we obtain the fourth order ODE

$$
\left\{\begin{array}{l}
-i \tilde{n} U_{s}^{\nu}\left(\partial_{y}^{2}-\tilde{n}^{2}\right) \phi_{n}+i \tilde{n} \partial_{y}^{2} U_{s}^{\nu} \phi_{n}+\nu\left(\partial_{y}^{2}-\tilde{n}^{2}\right)^{2} \phi_{n}=i \tilde{n} f_{n, 2}-\partial_{y} f_{n, 1}, \quad y>0 \\
\left.\phi_{n}\right|_{y=0}=\left.\partial_{y} \phi_{n}\right|_{y=0}=0 .
\end{array}\right.
$$


One can check that this fourth order ODE is equivalent to (1.13), and in particular, if $\phi_{n} \in$ $H^{4}\left(\mathbb{R}_{+}\right)$is a solution to this ODE then the velocity $u_{n}$ defined by (4.10) solves (1.13) with a suitable pressure $p_{n} \in H^{1}\left(\mathbb{R}_{+}\right)$. Next we introduce the rescaled variable $Y=y / \sqrt{\nu}$ and set

$$
\sqrt{\nu} \phi(Y)=\phi_{n}(y), \quad \frac{1}{\sqrt{\nu}} f(Y)=f_{n}(y)
$$

for simplicity. The rescaled unknown then satisfies

$$
-i \tilde{n} U_{s}\left(\partial_{Y}^{2}-\alpha^{2}\right) \phi+i \tilde{n} U_{s}^{\prime \prime} \phi+\left(\partial_{Y}^{2}-\alpha^{2}\right)^{2} \phi=i \tilde{n} f_{2}-\frac{1}{\sqrt{\nu}} \partial_{Y} f_{1}, \quad Y>0,
$$

where $U_{s}^{\prime \prime}=\partial_{Y}^{2} U_{s}$ and we have set

$$
\alpha=\tilde{n} \sqrt{\nu} .
$$

By dividing by $-i \tilde{n}$ of the above equation for $\phi$ and by setting $\varepsilon=1 / \tilde{n}$, we have arrived at the Orr-Sommerfeld equation

$$
\left\{\begin{array}{l}
U_{s}\left(\partial_{Y}^{2}-\alpha^{2}\right) \phi-U_{s}^{\prime \prime} \phi+i \varepsilon\left(\partial_{Y}^{2}-\alpha^{2}\right)^{2} \phi=-f_{2}-\frac{i}{\alpha} \partial_{Y} f_{1}, \quad Y>0 \\
\left.\phi\right|_{Y=0}=\left.\partial_{Y} \phi\right|_{Y=0}=0
\end{array}\right.
$$

We note that the constant $\varepsilon=1 / \tilde{n}=\kappa / n$ is small if $|n| \geq 1$ and $\kappa>0$ is small. For simplicity of notations we set

$$
\|f\|=\|f\|_{L_{Y}^{2}}=\left(\int_{0}^{\infty}|f(Y)|^{2} d Y\right)^{\frac{1}{2}}
$$

\section{Rayleigh equation}

In this section we consider the Rayleigh equation in the rescaled variable, where the Rayleigh operator is defined as $\operatorname{Ray}[\varphi]=U_{s}\left(\partial_{Y}^{2}-\alpha^{2}\right) \varphi-U_{s}^{\prime \prime} \varphi$. Without loss of generality we may assume $\alpha>0$. The system under study is

$$
\left\{\begin{array}{l}
\operatorname{Ray}[\varphi]=f, \quad Y>0 \\
\left.\varphi\right|_{Y=0}=0 .
\end{array}\right.
$$

Proposition 5.1. Let $f / U_{s} \in L^{2}\left(\mathbb{R}_{+}\right)$. Then there exists a unique solution $\varphi \in H^{2}\left(\mathbb{R}_{+}\right) \cap$ $H_{0}^{1}\left(\mathbb{R}_{+}\right)$to (5.1) such that

(i) when $\alpha \geq 1$,

$$
\begin{aligned}
\left\|\partial_{Y} \varphi\right\|+\alpha\|\varphi\| & \leq C \min \left\{\left\|\frac{Y}{U_{s}} f\right\|, \frac{1}{\alpha}\left\|\frac{f}{U_{s}}\right\|\right\}, \\
\left\|\left(\partial_{Y}^{2}-\alpha^{2}\right) \varphi\right\| & \leq C \min \left\{\left\|\frac{Y}{U_{s}} f\right\|, \frac{1}{\alpha}\left\|\frac{f}{U_{s}}\right\|\right\}+\left\|\frac{f}{U_{s}}\right\| .
\end{aligned}
$$

(ii) when $0<\alpha \leq 1$, if $(1+Y) \sigma[f] \in L^{2}\left(\mathbb{R}_{+}\right)$with $\sigma[f](Y)=\int_{Y}^{\infty} f d Y_{1}$ in addition,

$$
\begin{gathered}
\alpha\|\varphi\| \leq C \alpha\|(1+Y) \sigma[f]\|+\frac{C}{\alpha^{\frac{1}{2}}}\left|\int_{0}^{\infty} f d Y\right|, \\
\left\|\partial_{Y} \varphi\right\| \leq C(\|(1+Y) \sigma[f]\|+\|f\|)+\frac{C}{\alpha}\left|\int_{0}^{\infty} f d Y\right|, \\
\left\|\left(\partial_{Y}^{2}-\alpha^{2}\right) \varphi\right\| \leq C\left(\|(1+Y) \sigma[f]\|+\left\|\frac{f}{U_{s}}\right\|\right)+\frac{C}{\alpha}\left|\int_{0}^{\infty} f d Y\right| .
\end{gathered}
$$


Remark 5.2. (1) We obtain solutions even for $0<\alpha<1$, which is non-trivial. Note that the existence and uniqueness of $H^{2}$ solutions requires the condition $f / U_{s} \in L^{2}\left(\mathbb{R}_{+}\right)$. Nevertheless, it is seen from the proof that the existence and uniqueness of a weak solution in $H_{0}^{1}\left(\mathbb{R}_{+}\right)$is valid under the milder condition $f \in L^{2}\left(\mathbb{R}_{+}\right)$. In such a case, the only estimates that are retained are (i) when $\alpha \geq 1$,

$$
\left\|\partial_{Y} \varphi\right\|+\alpha\|\varphi\| \leq C\left\|\frac{Y}{U_{s}} f\right\|
$$

(ii) when $0<\alpha \leq 1$,

$$
\begin{aligned}
\alpha\|\varphi\| & \leq C \alpha\|(1+Y) \sigma[f]\|+\frac{C}{\alpha^{\frac{1}{2}}}\left|\int_{0}^{\infty} f d Y\right|, \\
\left\|\partial_{Y} \varphi\right\| & \leq C(\|(1+Y) \sigma[f]\|+\|f\|)+\frac{C}{\alpha}\left|\int_{0}^{\infty} f d Y\right| .
\end{aligned}
$$

Moreover, the solution is locally $H^{2}$ for $Y>0$, and satisfies

$$
\left\|U_{s}\left(\partial_{Y}^{2}-\alpha^{2}\right) \varphi\right\| \leq C\left\|\partial_{Y} \varphi\right\|+\|f\|,
$$

which is verified from the equation and the Hardy inequality $\left\|U_{s}^{\prime \prime} \varphi\right\| \leq C\left\|\partial_{Y} \varphi\right\|$, in virtue of the sufficient decay of $U_{s}^{\prime \prime}$ and $\left.\varphi\right|_{Y=0}=0$.

(2) The singularity in $\alpha$, seen in (5.4)-(5.5)-(5.6) when $0<\alpha \ll 1$, can not be avoided in general. It is due to the fact that $\alpha \rightarrow 0$ is a singular limit: indeed the formal limit system

$$
U_{s} \partial_{Y}^{2} \varphi-U_{s}^{\prime \prime} \varphi=f
$$

has in general no solution satisfying $\left.\varphi\right|_{Y=0}=0$. However, as we will show below, it has a solution under the additional condition that $f$ has zero average. This explains that the singular factor $\alpha^{-\frac{1}{2}}$ or $\alpha^{-1}$ is only in front of $\left|\int_{0}^{\infty} f d Y\right|$. This will be used crucially in our analysis.

(3) Estimate (5.4) is optimal in view of local regularity; roughly speaking, the $L^{2}$ norm of $\varphi$ is estimated in terms of $H^{-1}$ norm of $f$. We note that the Marcinkiewicz interpolation theorem yields the bound $\|(1+Y) \sigma[f]\| \leq C\|(1+Y) Y f\|$ as stated in Proposition 2.2 (1).

The proof of Proposition 5.1 is a consequence of the next lemma. We consider the problem

$$
\left\{\begin{array}{l}
\left(\partial_{Y}^{2}-\alpha^{2}\right) \varphi-\frac{U_{s}^{\prime \prime}}{U_{s}} \varphi=h, \quad Y>0, \\
\left.\varphi\right|_{Y=0}=0 .
\end{array}\right.
$$

Lemma 5.3. For any $h \in L^{2}\left(\mathbb{R}_{+}\right)$there exists a unique solution $\varphi \in H^{2}\left(\mathbb{R}_{+}\right) \cap H_{0}^{1}\left(\mathbb{R}_{+}\right)$and satisfies

(i) when $\alpha \geq 1$,

$$
\begin{aligned}
\left\|\partial_{Y} \varphi\right\|+\alpha\|\varphi\| & \leq C \min \left\{\|Y h\|, \frac{1}{\alpha}\|h\|\right\}, \\
\left\|\left(\partial_{Y}^{2}-\alpha^{2}\right) \varphi\right\| & \leq C \min \left\{\|Y h\|, \frac{1}{\alpha}\|h\|\right\}+\|h\|,
\end{aligned}
$$

(ii) when $0<\alpha \leq 1$, if $(1+Y) \sigma\left[U_{s} h\right] \in L^{2}\left(\mathbb{R}_{+}\right)$with $\sigma\left[U_{s} h\right](Y)=\int_{Y}^{\infty} U_{s} h d Y_{1}$ in addition,

$$
\begin{gathered}
\alpha\|\varphi\| \leq C \alpha\left\|(1+Y) \sigma\left[U_{s} h\right]\right\|+\frac{C}{\alpha^{\frac{1}{2}}}\left|\int_{0}^{\infty} U_{s} h d Y\right|, \\
\left\|\partial_{Y} \varphi\right\| \leq C\left(\left\|(1+Y) \sigma\left[U_{s} h\right]\right\|+\left\|U_{s} h\right\|\right)+\frac{C}{\alpha}\left|\int_{0}^{\infty} U_{s} h d Y\right|, \\
\left\|\left(\partial_{Y}^{2}-\alpha^{2}\right) \varphi\right\| \leq C\left(\left\|(1+Y) \sigma\left[U_{s} h\right]\right\|+\|h\|\right)+\frac{C}{\alpha}\left|\int_{0}^{\infty} U_{s} h d Y\right| .
\end{gathered}
$$


Proof. Let $T$ be the operator $T=\partial_{Y}^{2}-\alpha^{2}-U_{s}^{\prime \prime} / U_{s}$, which is realized in $L^{2}\left(\mathbb{R}_{+}\right)$with the domain $H^{2}\left(\mathbb{R}_{+}\right) \cap H_{0}^{1}\left(\mathbb{R}_{+}\right)$. Note that $T$ is relatively compact to $\partial_{Y}^{2}-\alpha^{2}$ whose domain is taken in the same way. This is because $\lim _{Y \rightarrow \infty} \frac{U_{s}^{\prime \prime}}{U_{s}}=0$ by our assumptions and because the Hardy inequality implies

$$
\left\|\frac{U_{s}^{\prime \prime}}{U_{s}} \varphi\right\| \leq\left\|\frac{Y U_{s}^{\prime \prime}}{U_{s}}\right\|_{L^{\infty}}\left\|\frac{\varphi}{Y}\right\| \leq 2\left\|\frac{Y U_{s}^{\prime \prime}}{U_{s}}\right\|_{L^{\infty}}\left\|\partial_{Y} \varphi\right\|, \quad \varphi \in H_{0}^{1}\left(\mathbb{R}_{+}\right),
$$

and thus the term $\frac{U_{s}^{\prime \prime}}{U_{s}} \varphi$ is a lower order operator both in view of regularity and spatial decay. Since the spectrum of $\partial_{Y}^{2}-\alpha^{2}$ is contained in $\left\{\lambda \in \mathbb{C} \mid \operatorname{Re} \lambda \leq-\alpha^{2}\right\}$ the spectrum of $T$ outside the set $\left\{\lambda \in \mathbb{C} \mid \operatorname{Re} \lambda \leq-\alpha^{2}\right\}$ consists only of isolated eigenvalues with finite multiplicities. Therefore, to show the invertiblity of $T$ in $L^{2}\left(\mathbb{R}_{+}\right)$it suffices to show the injectivity of $T$. To this end we observe the identity

$$
T \varphi=\frac{1}{U_{s}} \partial_{Y}\left(U_{s}^{2}\left(\partial_{Y}\left(\frac{\varphi}{U_{s}}\right)\right)-\alpha^{2} \varphi\right.
$$

and then, from the computation for $\varphi \in H^{2}\left(\mathbb{R}_{+}\right) \cap H_{0}^{1}\left(\mathbb{R}_{+}\right)$,

$$
\begin{aligned}
\lim _{\delta \downarrow 0} \int_{\delta}^{\infty} \frac{1}{U_{s}} \partial_{Y}\left(U_{s}^{2} \partial_{Y}\left(\frac{\varphi}{U_{s}}\right)\right) \bar{\varphi} d Y & =\lim _{\delta \downarrow 0}\left(-\int_{\delta}^{\infty} U_{s}^{2}\left|\partial_{Y}\left(\frac{\varphi}{U_{s}}\right)\right|^{2} d Y-\partial_{Y}\left(\frac{\varphi}{U_{s}}\right)(\delta) U_{s}(\delta) \bar{\varphi}(\delta)\right) \\
& =-\left\|U_{s} \partial_{Y}\left(\frac{\varphi}{U_{s}}\right)\right\|^{2},
\end{aligned}
$$

we verify the identity

$$
\left\|U_{s} \partial_{Y}\left(\frac{\varphi}{U_{s}}\right)\right\|^{2}+\alpha^{2}\|\varphi\|^{2}=-\operatorname{Re}\langle h, \varphi\rangle .
$$

Equality (5.14) implies the injectivity of $T$, and thus, $T$ is invertible in $L^{2}\left(\mathbb{R}_{+}\right)$as explained above. In particular, there exists a unique solution $\varphi \in H^{2}\left(\mathbb{R}_{+}\right) \cap H_{0}^{1}\left(\mathbb{R}_{+}\right)$to $T \varphi=h$.

Next we observe that the inner product with $\varphi$ in the equation $T \varphi=h$ also provides

$$
\left\|\partial_{Y} \varphi\right\|^{2}+\alpha^{2}\|\varphi\|^{2}=-\operatorname{Re}\left\langle\frac{U_{s}^{\prime \prime}}{U_{s}} \varphi, \varphi\right\rangle-\operatorname{Re}\langle h, \varphi\rangle .
$$

The first term in the right-hand side of (5.15) is estimated as

$$
\left|\left\langle\frac{U_{s}^{\prime \prime}}{U_{s}} \varphi, \varphi\right\rangle\right| \leq\left\|\frac{Y(1+Y) U_{s}^{\prime \prime}}{U_{s}}\right\|_{L^{2}}\left\|\frac{\varphi}{Y}\right\|\left\|\frac{1}{1+Y} \varphi\right\|_{L^{\infty}} \leq C\left\|\partial_{Y} \varphi\right\|\left\|\frac{\varphi}{1+Y}\right\|_{L^{\infty}} .
$$

Thus from (5.14) and (5.15), we obtain

$$
\begin{aligned}
\left\|U_{s} \partial_{Y}\left(\frac{\varphi}{U_{s}}\right)\right\|^{2}+\alpha^{2}\|\varphi\|^{2} & \leq|\operatorname{Re}\langle h, \varphi\rangle|, \\
\left\|\partial_{Y} \varphi\right\|^{2} & \leq C\left\|\frac{\varphi}{1+Y}\right\|_{L^{\infty}}^{2}+C|\operatorname{Re}\langle h, \varphi\rangle| .
\end{aligned}
$$

(i) When $\alpha \geq 1$ : In this case estimate (5.9) easily follows from (5.16) by applying $\left\|\frac{\varphi}{1+Y}\right\|_{L^{\infty}}^{2} \leq$ $C\|\varphi\|\left\|\partial_{Y} \varphi\right\|$. Finally, the estimate of $\left(\partial_{Y}^{2}-\alpha^{2}\right) \varphi$ is obtained from (5.8), for

$$
\left\|\left(\partial_{Y}^{2}-\alpha^{2}\right) \varphi\right\| \leq\left\|\frac{U_{s}^{\prime \prime}}{U_{s}} \varphi\right\|+\|h\| \leq 2\left\|\frac{Y U_{s}^{\prime \prime}}{U_{s}}\right\|_{L^{\infty}}\left\|\frac{\varphi}{Y}\right\|+\|h\| \leq C\left\|\partial_{Y} \varphi\right\|+\|h\| .
$$

The proof is complete. 
(ii) When $0<\alpha \leq 1$ : This case requires a more delicate analysis. We decompose $h$ as

$$
h=h_{1}+h_{2}, \quad h_{2}=\left(\int_{0}^{\infty} U_{s} h d Y\right) \rho, \quad \int_{0}^{\infty} U_{s} h_{1} d Y=0,
$$

where $\rho \in C_{0}^{\infty}\left(\mathbb{R}_{+}\right), \int_{0}^{\infty} U_{s} \rho d Y=1$, is taken independently of $\alpha$ and $h$, and we may assume that $\operatorname{supp} \rho \subset\{1 \leq Y \leq 2\}$. According to the decomposition of $h=h_{1}+h_{2}$ as above, we decompose $\varphi$ as $\varphi=\varphi_{1}+\varphi_{2}$, where

$$
T \varphi_{j}=h_{j},\left.\quad \varphi_{j}\right|_{Y=0}=0 .
$$

Step 1 (Estimate of $\varphi_{1}$ ). Let $\varphi_{1,1}$ be the function defined by

$$
\varphi_{1,1}=U_{s} \int_{Y}^{\infty} \frac{\sigma\left[U_{s} h_{1}\right]}{U_{s}^{2}} d Y_{1}=L\left[\sigma\left[U_{s} h_{1}\right]\right]
$$

where $L$ is studied in Proposition $2.2(2)$, and $\varphi_{1,1}$ satisfies $\frac{1}{U_{s}} \partial_{Y}\left(U_{s}^{2} \partial_{Y}\left(\frac{\varphi_{1,1}}{U_{s}}\right)\right)=h_{1}$ in $Y>0$. Moreover, in virtue of $\int_{0}^{\infty} U_{s} h_{1} d Y=0$, we can write $\sigma\left[U_{s} h_{1}\right]\left(Y_{1}\right)=-\int_{0}^{Y_{1}} U_{s} h_{1} d Y_{2}$, which ensure the boundary condition $\left.\varphi_{1,1}\right|_{Y=0}=0$ as well. From Proposition 2.2 (2) we have the estimate of $\varphi_{1,1}$ as follows.

$$
\left\|\varphi_{1,1}\right\|_{L^{2}}=\left\|L\left[\sigma\left[U_{s} h_{1}\right]\right]\right\| \leq C\left\|(1+Y) \sigma\left[U_{s} h_{1}\right]\right\| .
$$

We look for the solution $\varphi_{1}$ of the form $\varphi_{1}=\varphi_{1,1}+\varphi_{1,2}$, and thus, $\varphi_{1,2}$ is the solution to $T \varphi_{1,2}=\alpha^{2} \varphi_{1,1}$ in $Y>0$ and $\left.\varphi_{1,2}\right|_{Y=0}=0$. From (5.16) we have $\left\|\varphi_{1,2}\right\|^{2} \leq C\left\|\varphi_{1,1}\right\|^{2}$, which gives

$$
\left\|\varphi_{1}\right\| \leq\left\|\varphi_{1,1}\right\|+\left\|\varphi_{1,2}\right\| \leq C\left\|(1+Y) \sigma\left[U_{s} h_{1}\right]\right\| .
$$

Then, since $T \varphi_{1}=h_{1}$, we have again from (5.16) and $h_{1}=-\frac{1}{U_{s}} \partial_{Y} \sigma\left[U_{s} h_{1}\right]$ and $\left.\sigma\left[U_{s} h_{1}\right]\right|_{Y=0}=$ $\int_{0}^{\infty} U_{s} h d Y=0$

$$
\left\|U_{s} \partial_{Y}\left(\frac{\varphi_{1}}{U_{s}}\right)\right\|^{2} \leq\left|\operatorname{Re}\left\langle h_{1}, \varphi_{1}\right\rangle\right| \leq\left\|\frac{\sigma\left[U_{s} h_{1}\right]}{U_{s}}\right\|\left\|U_{s} \partial_{Y}\left(\frac{\varphi_{1}}{U_{s}}\right)\right\|
$$

that is, $\left\|U_{s} \partial_{Y}\left(\frac{\varphi_{1}}{U_{s}}\right)\right\| \leq\left\|\frac{\sigma\left[U_{s} h_{1}\right]}{U_{s}}\right\|$, and thus, (5.16) yields

$$
\begin{aligned}
\left\|\partial_{Y} \varphi_{1}\right\|^{2} \leq C\left\|\partial_{Y} \varphi_{1}\right\|\left\|\varphi_{1}\right\|+C\left|\operatorname{Re}\left\langle h_{1}, \varphi_{1}\right\rangle\right| & \leq C\left\|\varphi_{1}\right\|^{2}+C\left\|\frac{\sigma\left[U_{s} h_{1}\right]}{U_{s}}\right\|\left\|U_{s} \partial_{Y}\left(\frac{\varphi_{1}}{U_{s}}\right)\right\| \\
& \leq C\left\|(1+Y) \sigma\left[U_{s} h_{1}\right]\right\|^{2}+\left\|\frac{\sigma\left[U_{s} h_{1}\right]}{U_{s}}\right\|^{2} \\
& \leq C\left\|(1+Y) \sigma\left[U_{s} h_{1}\right]\right\|^{2}+C\left\|U_{s} h_{1}\right\|^{2} .
\end{aligned}
$$

Here we have used the Hardy-type inequality in the last line: $\left\|\frac{\sigma\left[U_{s} h_{1}\right]}{U_{s}}\right\| \leq C\left(\left\|\partial_{Y} \sigma\left[U_{s} h_{1}\right]\right\|+\right.$ $\left.\left\|\sigma\left[U_{s} h_{1}\right]\right\|\right)$. The $H^{2}$ estimate is then obtained from the equation and (5.21) as

$$
\begin{aligned}
\left\|\left(\partial_{Y}^{2}-\alpha^{2}\right) \varphi_{1}\right\| \leq\left\|\frac{U_{s}^{\prime \prime}}{U_{s}} \varphi_{1}\right\|+\left\|h_{1}\right\| & \leq C\left\|\partial_{Y} \varphi_{1}\right\|+\left\|h_{1}\right\| \\
& \leq C\left\|(1+Y) \sigma\left[U_{s} h_{1}\right]\right\|+C\left\|U_{s} h_{1}\right\|+\left\|h_{1}\right\| .
\end{aligned}
$$


Step 2 (Estimate of $\varphi_{2}$ ). Next we consider the estimate of $\varphi_{2}$, which is the solution to $T \varphi_{2}=h_{2}$ with $\left.\varphi_{2}\right|_{Y=0}=0$, where $h_{2}=\left(\int_{0}^{\infty} U_{s} h d Y\right) \rho$. First we set

$$
\begin{aligned}
& \varphi_{2,1,1}=U_{s} \int_{Y}^{\infty} \frac{1}{U_{s}^{2}} \int_{Y_{1}}^{\infty} U_{s} h_{2} d Y_{2} d Y_{1}=L\left[\sigma\left[U_{s} \rho\right]\right] \int_{0}^{\infty} U_{s} h d Y, \\
& \varphi_{2,1,2}=-U_{s} \int_{Y}^{Y_{0}} \frac{1}{U_{s}^{2}} d Y_{1} e^{-\alpha Y} \int_{0}^{\infty} U_{s} h d Y=-G_{s} e^{-\alpha Y} \int_{0}^{\infty} U_{s} h d Y .
\end{aligned}
$$

Here the function $G_{s}$ is studied in Proposition 2.1. Then, since $\rho \in C_{0}^{\infty}\left(\mathbb{R}_{+}\right)$, Proposition 2.2 (2) for $L$ and Proposition 2.1 (2) for $G_{s}$ imply

$$
\left\|\partial_{Y} \varphi_{2,1,1}\right\|+\left\|\varphi_{2,1,1}\right\|+\left\|\varphi_{2,1,1}\right\|_{L^{\infty}}+\left\|\frac{\varphi_{2,1,2}}{1+Y}\right\|_{L^{\infty}} \leq C\left|\int_{0}^{\infty} U_{s} h d Y\right| .
$$

Here $C$ is independent of $\alpha$. Moreover, the same computation as in the proof of Proposition 2.1 (2) leads to $\varphi_{2,1,1}(0)=\frac{1}{U_{s}^{\prime}(0)} \int_{0}^{\infty} U_{s} h d Y$ thanks to $\int_{0}^{\infty} U_{s} \rho d Y=1$, and thus, we have

$$
\left.\left(\varphi_{2,1,1}+\varphi_{2,1,2}\right)\right|_{Y=0}=0 .
$$

In particular, together with the estimates in Propositions 2.1 and 2.2 we see that $\varphi_{2,1}=$ $\varphi_{2,1,1}+\varphi_{2,1,2}$ belongs to $H_{0}^{1}\left(\mathbb{R}_{+}\right)$. Moreover, $\varphi_{2,1}$ satisfies for $Y>0$,

$$
\begin{aligned}
T \varphi_{2,1} & =h_{2}+2 \alpha \partial_{Y} G_{s} e^{-\alpha Y} \int_{0}^{\infty} U_{s} h d Y-\alpha^{2} \varphi_{2,1,1} \\
& =: h_{2}+g_{1} .
\end{aligned}
$$

To correct the error term $g_{1}$ we take $\varphi_{2,2}$ as the solution to $T \varphi_{2,2}=-g_{1}$ with $\left.\varphi_{2,2}\right|_{Y=0}=0$. Let us decompose $g_{1}$ as $g_{1}=g_{1} \chi+g_{1}(1-\chi)$, where $\chi$ is a smooth cut-off such that $\chi=1$ for $0 \leq Y \leq 1$ and $\chi=0$ for $Y \geq 2$, and let $\varphi_{2,2,1}$ and $\varphi_{2,2,2}$ be respectively the solutions in $H^{2}\left(\mathbb{R}_{+}\right) \cap H_{0}^{1}\left(\mathbb{R}_{+}\right)$to $T \varphi_{2,2,1}=-g_{1} \chi$ and $T \varphi_{2,2,2}=-g_{1}(1-\chi)$. From the formula $T=\frac{1}{U_{s}} \partial_{Y}\left(U_{s}^{2} \partial_{Y}\left(\frac{\dot{U_{s}}}{)}\right)\right)-\alpha^{2}$ we have the estimate

$$
\left\|\frac{\varphi_{2,2,2}}{U_{s}}\right\|_{L^{\infty}} \leq \frac{C}{\alpha^{2}}\left\|\frac{g_{1}(1-\chi)}{U_{s}}\right\|_{L^{\infty}} \leq \frac{C}{\alpha^{2}}\left\|g_{1}(1-\chi)\right\|_{L^{\infty}} .
$$

Indeed, for $l \in \mathbb{N}$ we compute $\left\langle T \varphi_{2,2,2}, \varphi_{2,2,2}\left(\frac{\varphi_{2,2,2}}{U_{s}}\right)^{2 l}\right\rangle=-\left\langle g_{1}(1-\chi), \varphi_{2,2,2}\left(\frac{\varphi_{2,2,2}}{U_{s}}\right)^{2 l}\right\rangle$, which gives

$$
\begin{aligned}
& (2 l+1) \int_{0}^{\infty}\left|\partial_{Y}\left(\frac{\varphi_{2,2,2}}{U_{s}}\right)\right|^{2}\left|\frac{\varphi_{2,2,2}}{U_{s}}\right|^{2 l} U_{s}^{2} d Y+\alpha^{2} \int_{0}^{\infty}\left|\frac{\varphi_{2,2,2}}{U_{s}}\right|^{2(l+1)} U_{s}^{2} d Y \\
& \leq \int_{0}^{\infty}\left|\frac{g_{1}(1-\chi)}{U_{s}}\right|\left|\frac{\varphi_{2,2,2}}{U_{s}}\right|^{2 l+1} U_{s}^{2} d Y,
\end{aligned}
$$

which gives

$$
\left(\int_{0}^{\infty}\left|\frac{\varphi_{2,2,2}}{U_{s}}\right|^{2(l+1)} U_{s}^{2} d Y\right)^{\frac{1}{2(l+1)}} \leq \frac{1}{\alpha^{2}}\left(\int_{0}^{\infty}\left|\frac{g_{1}(1-\chi)}{U_{s}}\right|^{2(l+1)} U_{s}^{2} d Y\right)^{\frac{1}{2(l+1)}} .
$$

Then taking the limit $l \rightarrow \infty$ yields (5.25). Thus we have from (5.24) and from Proposition 2.1 (2) for the $L^{\infty}$ bound of $\partial_{Y} G_{s}$ in $Y \geq 1$,

$$
\left\|\frac{\varphi_{2,2,2}}{U_{s}}\right\|_{L^{\infty}} \leq \frac{C}{\alpha}\left|\int_{0}^{\infty} U_{s} h d Y\right| .
$$


As for $\varphi_{2,2,1}$, we have from (5.16) that

$$
\left\|\varphi_{2,2,1}\right\| \leq \frac{C}{\alpha^{2}}\left\|g_{1} \chi\right\| \leq \frac{C}{\alpha}\left|\int_{1}^{2} U_{s} h_{2} d Y\right|,
$$

and thus, again from (5.16) and the interpolation inequality we have

$$
\left\|\partial_{Y} \varphi_{2,2,1}\right\|^{2} \leq C\left\|\varphi_{2,2,1}\right\|^{2}+C\left\|Y g_{1} \chi\right\|^{2} \leq \frac{C}{\alpha^{2}}\left|\int_{0}^{\infty} U_{s} h d Y\right| .
$$

Collecting these, we obtain

$$
\left\|\varphi_{2,2}\right\|_{L^{\infty}} \leq\left\|\varphi_{2,2,1}\right\|_{L^{\infty}}+\left\|\varphi_{2,2,2}\right\|_{L^{\infty}} \leq \frac{C}{\alpha}\left|\int_{0}^{\infty} U_{s} h d Y\right| .
$$

Thus, $\varphi_{2}=\varphi_{2,1}+\varphi_{2,2}=\varphi_{2,1,1}+\varphi_{2,1,2}+\varphi_{2,2}$ satisfies, by (5.24),

$$
\left\|\frac{\varphi_{2}}{1+Y}\right\|_{L^{\infty}} \leq \frac{C}{\alpha}\left|\int_{0}^{\infty} U_{s} h d Y\right| .
$$

Then the fact $T \varphi_{2}=h_{2}$ with $\left.\varphi_{2}\right|_{Y=0}=0$ and (5.16) with (5.27) yield

$$
\left\|\partial_{Y} \varphi_{2}\right\|^{2} \leq \frac{C}{\alpha^{2}}\left|\int_{0}^{\infty} U_{s} h d Y\right|^{2}+C\left\|Y h_{2}\right\|^{2} \leq \frac{C}{\alpha^{2}}\left|\int_{0}^{\infty} U_{s} h d Y\right|^{2},
$$

and therefore, again from (5.16),

$$
\alpha^{2}\left\|\varphi_{2}\right\|^{2} \leq C\left\|Y h_{2}\right\|\left\|\partial_{Y} \varphi_{2}\right\| \leq \frac{C}{\alpha}\left|\int_{0}^{\infty} U_{s} h d Y\right|^{2} .
$$

Finally the $H^{2}$ estimate of $\varphi_{2}$ is obtained from the equation as

$$
\left\|\left(\partial_{Y}^{2}-\alpha^{2}\right) \varphi_{2}\right\| \leq\left\|\frac{U_{s}^{\prime \prime}}{U_{s}} \varphi_{2}\right\|+\left\|h_{2}\right\| \leq C\left\|\partial_{Y} \varphi_{2}\right\|+\left\|h_{2}\right\| \leq \frac{C}{\alpha}\left|\int_{0}^{\infty} U_{s} h d Y\right| .
$$

Collecting (5.20), (5.21), (5.22) with $h_{1}=h-\left(\int_{0}^{\infty} U_{s} h d Y\right) \rho$, and (5.28), (5.29), and (5.30), we obtain the estimates of $\varphi=\varphi_{1}+\varphi_{2}$. The proof is complete.

The next proposition will be used to construct a slow mode in the case $0<\alpha \ll 1$, the boundary corrector for the Orr-Sommerfeld equation. For later use let us introduce the operator

$$
K[f](Y)=U_{s} \int_{Y}^{\infty} \frac{1}{U_{s}^{2}} \int_{Y_{1}}^{\infty} f d Y_{2} d Y_{1}=L[\sigma[f]](Y), \quad f \in C_{0}^{\infty}\left(\mathbb{R}_{+}\right) .
$$

The estimate of $K$ will be derived from the estimates of $L$ and $\sigma$ in Proposition 2.2.

Proposition 5.4. For any $0<\alpha \leq 1$, there exists a function $\varphi \in H^{1}\left(\mathbb{R}_{+}\right)$satisfying

$$
\operatorname{Ray}[\varphi]=0 \quad Y>0
$$

in the sense of distributions and the following properties: $\varphi=\varphi_{0}+\varphi_{1}+\varphi_{2}$, where

$$
\begin{aligned}
& \varphi_{0}=U_{s} e^{-\alpha Y},\left.\quad \varphi_{1}\right|_{Y=0}=\frac{U_{E}^{2}}{U_{s}^{\prime}(0)} \alpha+O\left(\alpha^{2}\right), \\
& \left\|\partial_{Y} \varphi_{1}\right\|+\left\|\varphi_{1}\right\| \leq C \alpha \\
& \left\|\partial_{Y} \varphi_{2}\right\|+\alpha\left\|\varphi_{2}\right\| \leq C \alpha^{3 / 2} .
\end{aligned}
$$

Here $C$ is independent of $\alpha$. If $\frac{U_{s}^{\prime \prime}}{U_{s}} \in L^{2}\left(\mathbb{R}_{+}\right)$in addition, then $\varphi_{1}$ and $\varphi_{2}$ belong to $H^{2}\left(\mathbb{R}_{+}\right)$. 
Proof. We look for a solution $\varphi$ to $\operatorname{Ray}[\varphi]=0$ of the form $\varphi=U_{s} e^{-\alpha Y}+\varphi_{1}+\varphi_{2}$. We should have $\operatorname{Ray}\left[\varphi_{1}+\varphi_{2}\right]=2 \alpha U_{s} U_{s}^{\prime} e^{-\alpha Y}$.

We first consider the problem

$$
U_{s} \partial_{Y}^{2} \varphi_{1}-U_{s}^{\prime \prime} \varphi_{1}=2 \alpha U_{s} U_{s}^{\prime} e^{-\alpha Y}, \quad \lim _{Y \rightarrow \infty} \varphi_{1}=0
$$

Its solution is given by

$$
\varphi_{1}=2 \alpha K\left[U_{s} U_{s}^{\prime} e^{-\alpha Y}\right],
$$

where $K$ is defined by (5.31). From Proposition 2.2 we have

$$
\begin{aligned}
\left\|\varphi_{1}\right\|=2 \alpha\left\|L\left[\sigma\left[U_{s} U_{s}^{\prime} e^{-\alpha Y}\right]\right]\right\| & \leq C \alpha\left\|(1+Y) \sigma\left[U_{s} U_{s}^{\prime} e^{-\alpha Y}\right]\right\| \\
& \leq C \alpha\left(\left\|(1+Y)^{2} U_{s} U_{s}^{\prime} e^{-\alpha Y}\right\| \leq C \alpha .\right.
\end{aligned}
$$

Here we have used the decay condition $\left|U_{s}^{\prime}(Y)\right| \leq C(1+Y)^{-3}$. Similarly, we have

$$
\begin{aligned}
\left\|\partial_{Y} \varphi_{1}\right\| & \leq C \alpha\left(\left\|\sigma\left[U_{s} U_{s}^{\prime} e^{-\alpha Y}\right]\right\|_{L^{1}}+\left\|\sigma\left[U_{s} U_{s}^{\prime} e^{-\alpha Y}\right]\right\|+\left\|\partial_{Y} \sigma\left[U_{s} U_{s}^{\prime} e^{-\alpha Y}\right]\right\|\right) \\
& \leq C \alpha\left(\left\|Y U_{s} U_{s}^{\prime} e^{-\alpha Y}\right\|_{L^{1}}+\left\|Y U_{s} U_{s}^{\prime} e^{-\alpha Y}\right\|+\left\|U_{s} U_{s}^{\prime} e^{-\alpha Y}\right\|\right) \\
& \leq C \alpha
\end{aligned}
$$

Estimate (5.33) is proved.

Eventually, we introduce the solution $\varphi_{2}$ of

$$
\operatorname{Ray}\left[\varphi_{2}\right]=\alpha^{2} U_{s} \varphi_{1}=2 \alpha^{3} U_{s} K\left[U_{s} U_{s}^{\prime} e^{-\alpha Y}\right],\left.\quad \varphi_{2}\right|_{Y=0}=0,
$$

that can be estimated using case ii) of Proposition 5.1. We use again Proposition 2.2 and the bound $\left|U_{s}^{\prime}(Y)\right| \leq C(1+Y)^{-3}$ to compute:

$$
\begin{aligned}
\left\|\partial_{Y} \varphi_{2}\right\|+\alpha\left\|\varphi_{2}\right\| & \leq C \alpha^{3}\left\|(1+Y)^{2} K\left[U_{s} U_{s}^{\prime} e^{-\alpha Y}\right]\right\|+C \alpha^{2}\left|\int_{0}^{+\infty} K\left[U_{s} U_{s}^{\prime} e^{-\alpha Y}\right](Y) d Y\right| \\
& \leq C \alpha^{3}\left\|(1+Y)^{4} U_{s} U_{s}^{\prime} e^{-\alpha Y}\right\|+C \alpha^{2} \int_{0}^{+\infty}(1+Y)^{-1} e^{-\alpha Y} d Y \\
& \leq C \alpha^{3}\left\|(1+Y) e^{-\alpha Y}\right\|+C \alpha^{2}|\ln \alpha| \leq C \alpha^{3 / 2}+\alpha^{2}|\ln \alpha| .
\end{aligned}
$$

Here, note that the bound $\left|K\left[U_{s} U_{s}^{\prime} e^{-\alpha Y}\right](Y)\right| \leq C(1+Y)^{-1} e^{-\alpha Y}$ used in the second line is proved by the following observation:

$$
\begin{aligned}
\left|K\left[U_{s} U_{s}^{\prime} e^{-\alpha Y}\right](Y)\right| & \leq C U_{s} e^{-\alpha Y} \int_{Y}^{\infty} \frac{1}{U_{s}^{2}} \int_{Y_{1}}^{\infty}\left(1+Y_{2}\right)^{-3} d Y_{2} d Y_{1} \\
& \leq C U_{s} e^{-\alpha Y} \int_{Y}^{\infty} \frac{1}{U_{s}^{2}\left(1+Y_{1}\right)^{2}} d Y_{1} \leq C(1+Y) e^{-\alpha Y}
\end{aligned}
$$

In the last line we have also used the argument as in the computation of $G_{s}$ in Proposition 2.1 (2) when $Y$ is small. The details are omitted here. The proof is complete.

Let $\varphi=\varphi_{0}+\varphi_{1}+\varphi_{2} \in H^{1}\left(\mathbb{R}_{+}\right)$be the function obtained in Proposition 5.4, and set

$$
\varphi_{\text {slow }, \text { Ray }}=\frac{c_{E}}{\alpha} \varphi, \quad c_{E}=\frac{\alpha}{\varphi_{1}(0)}=\frac{U_{s}^{\prime}(0)}{U_{E}^{2}}+O(\alpha), \quad 0<\alpha \leq 1
$$

As a direct consequence of Proposition 5.4, we have 
Corollary 5.5. For any $0<\alpha \leq 1$, there exists a function $\varphi_{\text {slow, Ray }} \in H^{1}\left(\mathbb{R}_{+}\right)$satisfying

$$
\operatorname{Ray}\left[\varphi_{\text {slow }, R a y}\right]=0 \quad Y>0
$$

in the sense of distributions and the following properties: $\varphi_{\text {slow, Ray }}=\varphi_{\text {sRay }, 0}+\varphi_{\text {sRay }, 1}+$ $\varphi_{\text {sRay }, 2}$, where

$$
\begin{aligned}
& \varphi_{\text {sRay }, 0}=\frac{c_{E}}{\alpha} U_{s} e^{-\alpha Y}, \quad \varphi_{\text {sRay }, 1}(0)=1, \\
& \left\|\partial_{Y} \varphi_{s R a y, 1}\right\|+\left\|\varphi_{\text {sRay }, 1}\right\| \leq C \\
& \left\|\partial_{Y} \varphi_{\text {sRay }, 2}\right\|+\alpha\left\|\varphi_{\text {sRay }, 2}\right\| \leq C \alpha^{1 / 2}
\end{aligned}
$$

Here $C$ is independent of $\alpha$. In particular, we have

$$
\varphi_{\text {slow, Ray }}(0)=1
$$

If $\frac{U_{s}^{\prime \prime}}{U_{s}} \in L^{2}\left(\mathbb{R}_{+}\right)$in addition, then $\varphi_{\text {sRay }, 1}$ and $\varphi_{\text {sRay }, 2}$ belong to $H^{2}\left(\mathbb{R}_{+}\right)$.

When $\alpha \geq 1$ we have

Proposition 5.6. If $\alpha \geq 1$ then there exists a function $\varphi_{\text {slow, Ray }} \in H^{1}\left(\mathbb{R}_{+}\right)$satisfying

$$
\operatorname{Ray}\left[\varphi_{\text {slow }, \text { Ray }}\right]=0 \quad Y>0,\left.\quad \varphi_{\text {slow, Ray }}\right|_{Y=0}=1,
$$

and the following properties: $\varphi_{\text {slow, Ray }}=e^{-\alpha Y}+\tilde{\varphi}_{\text {slow, Ray }}$ with $\tilde{\varphi}_{\text {slow, Ray }} \in H_{0}^{1}\left(\mathbb{R}_{+}\right)$, where

$$
\left\|\partial_{Y} \tilde{\varphi}_{\text {slow }, \text { Ray }}\right\|+\alpha\left\|\tilde{\varphi}_{\text {slow,Ray }}\right\| \leq C \min \left\{1, \alpha^{-\frac{1}{2}}\right\} .
$$

Here $C$ is independent of $\alpha \geq 1$. If $\frac{U_{s}^{\prime \prime}}{U_{s}} \in L^{2}\left(\mathbb{R}_{+}\right)$in addition, then $\tilde{\varphi}_{\text {slow, Ray belongs to }}$ $H^{2}\left(\mathbb{R}_{+}\right)$.

Proof. The function $\tilde{\varphi}_{\text {slow, Ray }}$ is constructed as the weak solution to

$$
\operatorname{Ray}\left[\tilde{\varphi}_{\text {slow }, \text { Ray }}\right]=U_{s}^{\prime \prime} e^{-\alpha Y},\left.\quad \tilde{\varphi}_{\text {slow }, R a y}\right|_{Y=0}=0 .
$$

Proposition 5.1 shows that

$$
\left\|\partial_{Y} \tilde{\varphi}_{\text {slow }, R a y}\right\|+\alpha\left\|\tilde{\varphi}_{\text {slow }, \text { Ray }}\right\| \leq C\left\|(1+Y) U_{s}^{\prime \prime} e^{-\alpha Y}\right\| \leq C \min \left\{1, \alpha^{-\frac{1}{2}}\right\} .
$$

From Ray $=U_{s}\left(\partial_{Y}^{2}-\alpha^{2}\right)-U_{s}^{\prime \prime}$ it is straightforward that $\tilde{\varphi}_{\text {slow, Ray }}$ belongs to $H^{2}\left(\mathbb{R}_{+}\right)$if $\frac{U_{s}^{\prime \prime}}{U_{s}} \in L^{2}\left(\mathbb{R}_{+}\right)$. The proof is complete.

\section{Airy equation}

Set $\operatorname{Airy}[\psi]=U_{s} \psi+i \varepsilon\left(\partial_{Y}^{2}-\alpha^{2}\right) \psi$ with $\varepsilon=1 / \tilde{n}$. In this section we consider the Airy equation

$$
\left\{\begin{array}{l}
\operatorname{Airy}[\psi]=\varepsilon f, \quad Y>0, \\
\left.\psi\right|_{Y=0}=0 .
\end{array}\right.
$$

Proposition 6.1. Let $f \in L^{2}\left(\mathbb{R}_{+}\right)$. Then there exists a unique solution $\psi \in H^{2}\left(\mathbb{R}_{+}\right) \cap H_{0}^{1}\left(\mathbb{R}_{+}\right)$ to (6.1) such that

$$
\left\|U_{s} \psi\right\|+\varepsilon^{\frac{1}{6}}\left\|\sqrt{U_{s}} \psi\right\|+\varepsilon^{\frac{1}{3}}\|\psi\|+\varepsilon^{\frac{2}{3}}\left(\left\|\partial_{Y} \psi\right\|+\alpha\|\psi\|\right)+\varepsilon\left\|\left(\partial_{Y}^{2}-\alpha^{2}\right) \psi\right\| \leq C \varepsilon\|f\|,
$$


and also

$$
\left\|U_{s} Y \psi\right\| \leq C \varepsilon\|Y f\|+C \varepsilon^{\frac{4}{3}}\|f\|
$$

if $(1+Y) f \in L^{2}\left(\mathbb{R}_{+}\right)$in addition. Moreover, if $f$ is replaced by $\partial_{Y} f$ or $\frac{f}{Y}$, then

$$
\varepsilon^{\frac{1}{2}}\left\|\sqrt{U_{s}} \psi\right\|+\varepsilon^{\frac{2}{3}}\|\psi\|+\varepsilon\left(\left\|\partial_{Y} \psi\right\|+\alpha\|\psi\|\right) \leq C \varepsilon\|f\|
$$

In the case when $f$ is replaced by $\frac{f}{Y}$ we also have

$$
\left\|U_{s} \psi\right\| \leq C \varepsilon^{\frac{2}{3}}\|f\|
$$

Remark 6.2. From the proof one can check that the unique existence of the weak solution in $H_{0}^{1}\left(\mathbb{R}_{+}\right)$is valid even when $f$ is replaced by $\partial_{Y} f$ or $\frac{f}{Y}$.

Proof. We focus on the a priori estimates. We first take the inner product with $\psi$ in the equation $\operatorname{Airy}[\psi]=\varepsilon f$, and then the real part and the imaginary part respectively give

$$
\begin{aligned}
\left\|\sqrt{U_{s}} \psi\right\|^{2} & =\varepsilon \operatorname{Re}\langle f, \psi\rangle, \\
\left\|\partial_{Y} \psi\right\|^{2}+\alpha^{2}\|\psi\|^{2} & =-\mathcal{I} m\langle f, \psi\rangle .
\end{aligned}
$$

Similarly, we take the inner product with $\left(\partial_{Y}^{2}-\alpha^{2}\right) \psi$ in the equation Airy $[\psi]=\varepsilon f$, and then the imaginary part lead to

$$
\varepsilon\left\|\left(\partial_{Y}^{2}-\alpha^{2}\right) \psi\right\|^{2}=\mathcal{I} m\left\langle U_{s}^{\prime} \psi, \partial_{Y} \psi\right\rangle+\varepsilon\left\langle f,\left(\partial_{Y}^{2}-\alpha^{2}\right) \psi\right\rangle .
$$

To obtain the estimate of $\|\psi\|$, we apply the interpolation inequality (2.1) together with (6.6)-(6.7). They imply

$$
\begin{aligned}
\|\psi\|^{2} & \leq C\left\|\sqrt{U_{s}} \psi\right\|^{\frac{4}{3}}\left\|\partial_{Y} \psi\right\|^{\frac{2}{3}}+C\left\|\sqrt{U_{s}} \psi\right\|^{2} \\
& \leq C(\varepsilon \operatorname{Re}\langle f, \psi\rangle)^{\frac{2}{3}}(|\mathcal{I} m\langle f, \psi\rangle|)^{\frac{1}{3}}+C \varepsilon \operatorname{Re}\langle f, \psi\rangle \\
& \leq C \varepsilon^{\frac{2}{3}}\|f\|\|\psi\|+\varepsilon\|f\|\|\psi\| \\
& \leq C \varepsilon^{\frac{4}{3}}\|f\|^{2} .
\end{aligned}
$$

Here $C$ is a universal constant. This proves the estimate of $\|\psi\|$. The $H^{1}$ estimate of $\psi$ then follows from the estimate of $\|\psi\|$ and (6.7). The $H^{2}$ estimate easily follows from (6.8) and the $H^{1}$ estimate of $\psi$, while the estimate $\left\|\sqrt{U_{s}} \psi\right\| \leq C \varepsilon^{\frac{5}{6}}\|f\|$ is obtained from the estimate of $\|\psi\|$ and (6.6). The details are omitted here. Next we take the inner product with $U_{s} \psi$ in the equation Airy $[\psi]=\varepsilon f$ and take the real part, which gives

$$
\left\|U_{s} \psi\right\|^{2}+\varepsilon \mathcal{I} m\left\langle\partial_{Y} \psi, U_{s}^{\prime} \psi\right\rangle=\varepsilon \operatorname{Re}\left\langle f, U_{s} \psi\right\rangle .
$$

Thus we have

$$
\left\|U_{s} \psi\right\|^{2} \leq \varepsilon\left\|U_{s}^{\prime}\right\|_{L^{\infty}}\left\|\partial_{Y} \psi\right\|\|\psi\|+\varepsilon\|f\|\left\|U_{s} \psi\right\| \leq C \varepsilon^{2}\|f\|^{2} .
$$

Hence the estimate of $\left\|U_{s} \psi\right\|$ holds. When $(1+Y) f \in L^{2}\left(\mathbb{R}_{+}\right)$it is not difficult to show that $(1+Y) \psi \in H^{2}\left(\mathbb{R}_{+}\right) \cap H_{0}^{1}\left(\mathbb{R}_{+}\right)$. Indeed, we first take the inner product with $Y^{2} \chi_{R}^{2} \psi$ to Airy $[\psi]=\varepsilon f$, where $\chi_{R}$ is a smooth cut-off such that $\chi_{R}=1$ for $0 \leq Y \leq R$ and $\chi_{R}=0$ for $Y \geq 2 R$ with $\left\|\partial_{Y}^{k} \chi_{R}\right\|_{L^{\infty}} \leq C R^{-k}$. Then taking the limit $R \rightarrow \infty$ verifies 
$Y \psi, Y \partial_{Y} \psi \in L^{2}\left(\mathbb{R}_{+}\right)$, from which it is also easy to see that $(1+Y) \psi \in H^{2}\left(\mathbb{R}_{+}\right)$by using the elliptic regularity. Now we observe that

$$
\operatorname{Airy}[Y \psi]=\varepsilon Y f+i 2 \varepsilon \partial_{Y} \psi .
$$

Thus we have

$$
\left\|U_{s} Y \psi\right\| \leq C \varepsilon\|Y f\|+2 \varepsilon\left\|\partial_{Y} \psi\right\| \leq C \varepsilon\|Y f\|+C \varepsilon^{\frac{4}{3}}\|f\| .
$$

This proves (6.3). Finally, let us consider the case when $f$ is replaced by $\partial_{Y} f$ or $\frac{f}{Y}$. We give the proof only for the case $\frac{f}{Y}$, for the argument of the case $\partial_{Y} f$ is the same by applying the integration by parts in the inner product $\left\langle\partial_{Y} f, \psi\right\rangle=-\left\langle f, \partial_{Y} \psi\right\rangle$. The energy equalities (6.6)-(6.7) are replaced by

$$
\begin{aligned}
\left\|\sqrt{U_{s}} \psi\right\|^{2} & =\varepsilon \operatorname{Re}\left\langle f, \frac{\psi}{Y}\right\rangle, \\
\left\|\partial_{Y} \psi\right\|^{2}+\alpha^{2}\|\psi\|^{2} & =-\mathcal{I} m\left\langle f, \frac{\psi}{Y}\right\rangle .
\end{aligned}
$$

Equality (6.10) gives the $H^{1}$ estimate by the Hardy inequality $\left\|\frac{\psi}{Y}\right\| \leq C\left\|\partial_{Y} \psi\right\|$, and then the estimate of $\left\|\sqrt{U_{s}} \psi\right\|$ follows from (6.9) and the estimate of $\left\|\partial_{Y} \psi\right\|$ by applying the Hardy inequality for the term $\frac{\psi}{Y}$ again. The estimate of $\|\psi\|$ then follows from the interpolation inequality (2.1) and the estimates of $\left\|\sqrt{U_{s}} \psi\right\|$ and $\left\|\partial_{Y} \psi\right\|$. Estimate (6.5) follows as above from the equality

$$
\left\|U_{s} \psi\right\|^{2}+\varepsilon \mathcal{I} m\left\langle\partial_{Y} \psi, U_{s}^{\prime} \psi\right\rangle=\varepsilon \operatorname{Re}\left\langle f, \frac{U_{s}}{Y} \psi\right\rangle,
$$

and by using the bound $\left\|\frac{U_{s}}{Y}\right\|_{L^{\infty}}<\infty$. The details are omitted. The uniqueness of the solution follows from the a priori estimates.

As for the existence, we first consider the problem Airy $[\psi]-i l \psi=f$ for $l>0$. When $l$ is large the operator Airy - $i l$ is clearly invertible in $L^{2}\left(\mathbb{R}_{+}\right)$, while all of the a priori estimates are valid uniformly in $l>0$ by applying the same argument as above. This implies the existence of the solution for the case $l=0$ by the standard continuity method. The proof is complete.

For later use we consider the Airy equation under the Neumann boundary condition:

$$
\left\{\begin{array}{l}
\operatorname{Airy}[\psi]=\partial_{Y} f, \quad Y>0, \\
\left.\partial_{Y} \psi\right|_{Y=0}=0 .
\end{array}\right.
$$

Proposition 6.3. Let $f \in H_{0}^{1}\left(\mathbb{R}_{+}\right)$. Then there exists a unique solution $\psi \in H^{2}\left(\mathbb{R}_{+}\right)$to (6.11) such that

$$
\left\|U_{s} \psi\right\| \leq \frac{C}{\varepsilon^{\frac{1}{3}}}\|f\|+\frac{C}{\varepsilon^{\frac{2}{3}}}\left\|U_{s} f\right\|,
$$

and

$$
\varepsilon^{\frac{1}{2}}\left\|\sqrt{U_{s}} \psi\right\|+\varepsilon^{\frac{2}{3}}\|\psi\|+\varepsilon\left(\left\|\partial_{Y} \psi\right\|+\alpha\|\psi\|\right) \leq C\|f\| .
$$

Moreover, if $(1+Y)^{2} f \in H^{1}\left(\mathbb{R}_{+}\right)$in addition, then

$$
\begin{aligned}
\|Y \psi\| & \leq \frac{C}{\varepsilon^{\frac{2}{3}}}\|Y f\|+\frac{C}{\varepsilon^{\frac{1}{3}}}\|f\|, \\
\left\|Y^{2} U_{s} \psi\right\| & \leq \frac{C}{\varepsilon^{\frac{2}{3}}}\left\|U_{s} Y^{2} f\right\|+\frac{C}{\varepsilon^{\frac{1}{3}}}\left\|U_{s} Y f\right\|+C\|Y f\|+C \varepsilon^{\frac{1}{3}}\|f\|,
\end{aligned}
$$


and also

$$
\begin{gathered}
\|\psi\|_{L^{1}} \leq \frac{C}{\epsilon^{\frac{5}{6}}}\|Y f\|+\frac{C}{\varepsilon^{\frac{1}{2}}}\|f\|, \\
\left|\int_{0}^{\infty} U_{s} \psi d Y\right| \leq C \alpha^{2}\left(\varepsilon^{\frac{1}{6}}\|Y f\|+\epsilon^{\frac{1}{2}}\|f\|\right) .
\end{gathered}
$$

Finally, when $0 \leq \alpha \leq 1$, the function $\sigma\left[U_{s} \psi\right](Y)=\int_{Y}^{\infty} U_{s} \psi d Y_{1}$ satisfies

$$
\left\|(1+Y) \sigma\left[U_{s} \psi\right]\right\| \leq C\left\|(1+Y)^{2} f\right\| .
$$

Proof. As in the proof of the previous proposition, we have

$$
\begin{array}{r}
\left\|\sqrt{U_{s}} \psi\right\|^{2}=-\operatorname{Re}\left\langle f, \partial_{Y} \psi\right\rangle, \\
\left\|\partial_{Y} \psi\right\|^{2}+\alpha^{2}\|\psi\|^{2}=\varepsilon^{-1} \mathcal{I} m\left\langle f, \partial_{Y} \psi\right\rangle,
\end{array}
$$

and also

$$
\varepsilon\left\|\left(\partial_{Y}^{2}-\alpha^{2}\right) \psi\right\|^{2}=\mathcal{I} m\left\langle U_{s}^{\prime} \psi, \partial_{Y} \psi\right\rangle+\left\langle f,\left(\partial_{Y}^{2}-\alpha^{2}\right) \psi\right\rangle .
$$

The interpolation inequality

$$
\|\psi\|^{2} \leq C\left\|\sqrt{U_{s}} \psi\right\|^{\frac{4}{3}}\left\|\partial_{Y} \psi\right\|^{\frac{2}{3}}+C\left\|\sqrt{U_{s}} \psi\right\|^{2}
$$

is valid for $\psi \in H^{1}\left(\mathbb{R}_{+}\right)$and thus, (6.19) and (6.20) imply

$$
\|\psi\|^{2} \leq \frac{C}{\varepsilon^{\frac{1}{3}}}\|f\|\left\|\partial_{Y} \psi\right\|+\|f\|\left\|\partial_{Y} \psi\right\| \leq \frac{C}{\varepsilon^{\frac{4}{3}}}\|f\|^{2} .
$$

Estimate (6.13) has been proved. Next by taking the inner product with $U_{s} \psi$ in the equation $\operatorname{Airy}[\psi]=\partial_{Y} f$ and by taking the real part, we see

$$
\left\|U_{s} \psi\right\|^{2}+\varepsilon \mathcal{I} m\left\langle\partial_{Y} \psi, U_{s}^{\prime} \psi\right\rangle=-\operatorname{Re}\left\langle f, \partial_{Y}\left(U_{s} \psi\right)\right\rangle
$$

Thus we have

$$
\begin{aligned}
\left\|U_{s} \psi\right\|^{2} & \leq \varepsilon\left\|U_{s}^{\prime}\right\|_{L^{\infty}}\left\|\partial_{Y} \psi\right\|\|\psi\|+C\|f\|\|\psi\|+\left\|U_{s} f\right\|\left\|\partial_{Y} \psi\right\| \\
& \leq \frac{C}{\varepsilon^{\frac{2}{3}}}\|f\|^{2}+\frac{C}{\varepsilon^{\frac{2}{3}}}\|f\|^{2}+\frac{C}{\varepsilon}\left\|U_{s} f\right\|\|f\| .
\end{aligned}
$$

Hence (6.12) holds. To obtain the weighted estimate we see

$$
\operatorname{Airy}[Y \psi]=Y \partial_{Y} f+2 i \varepsilon \partial_{Y} \psi=\partial_{Y}(Y f)-f+2 i \varepsilon \partial_{Y} \psi,
$$

and we have by applying Proposition 6.1,

$$
\begin{aligned}
\varepsilon^{\frac{1}{3}}\left\|\partial_{Y}(Y \psi)\right\|+\|Y \psi\| & \leq \frac{C}{\varepsilon^{\frac{2}{3}}}\|Y f\|+\frac{C}{\varepsilon^{\frac{1}{3}}}\|f\|+C \varepsilon^{\frac{1}{3}}\|\psi\| \\
& \leq \frac{C}{\varepsilon^{\frac{2}{3}}}\|Y f\|+\frac{C}{\varepsilon^{\frac{1}{3}}}\|f\| .
\end{aligned}
$$

Hence (6.14) holds. Moreover, from the computation of the inner product

$$
\begin{aligned}
\left\langle\operatorname{Airy}[Y \psi], U_{s} Y \psi\right\rangle & =\left\langle Y \partial_{Y} f+2 i \varepsilon \partial_{Y} \psi, U_{s} Y \psi\right\rangle \\
& =-\left\langle f, \partial_{Y}\left(Y U_{s} Y \psi\right)\right\rangle+2 i \varepsilon\left\langle\partial_{Y} \psi, U_{s} Y \psi\right\rangle \\
& =-\left\langle U_{s} Y f, \partial_{Y}(Y \psi)\right\rangle-\left\langle f, U_{s} Y \psi\right\rangle-\left\langle U_{s}^{\prime} Y f, Y \psi\right\rangle+2 i \varepsilon\left\langle\partial_{Y} \psi, U_{s} Y \psi\right\rangle
\end{aligned}
$$


and by taking the real part of it, we finally achieve from (6.22),

$$
\left\|U_{s} Y \psi\right\| \leq \frac{C}{\varepsilon^{\frac{1}{3}}}\|Y f\|+\frac{C}{\varepsilon^{\frac{2}{3}}}\left\|U_{s} Y f\right\|+C\|f\| .
$$

The details are omitted here. Next we see

$$
\operatorname{Airy}\left[Y^{2} \psi\right]=Y^{2} \partial_{Y} f+2 i \varepsilon \psi+4 i \varepsilon Y \partial_{Y} \psi=\partial_{Y}\left(Y^{2} f\right)-2 Y f-2 i \varepsilon \psi+4 i \varepsilon \partial_{Y}(Y \psi) .
$$

Thus from (6.12) and Proposition 6.1,

$$
\begin{aligned}
\left\|U_{s} Y^{2} \psi\right\| \leq & \frac{C}{\varepsilon^{\frac{1}{3}}}\left\|Y^{2} f\right\|+\frac{C}{\varepsilon^{\frac{2}{3}}}\left\|U_{s} Y^{2} f\right\|+C\|Y f\|+C \varepsilon\|\psi\| \\
& +C \varepsilon\left(\frac{1}{\varepsilon^{\frac{1}{3}}}\|Y \psi\|+\frac{1}{\varepsilon^{\frac{2}{3}}}\left\|U_{s} Y \psi\right\|\right) \\
\leq & \frac{C}{\varepsilon^{\frac{1}{3}}}\left\|Y^{2} f\right\|+\frac{C}{\varepsilon^{\frac{2}{3}}}\left\|U_{s} Y^{2} f\right\|+C\|Y f\|+C \varepsilon^{\frac{1}{3}}\|f\| \\
& +C \varepsilon^{\frac{2}{3}}\|Y \psi\|+C \varepsilon^{\frac{1}{3}}\left\|U_{s} Y \psi\right\| .
\end{aligned}
$$

Next we have from the interpolation $\|\psi\|_{L^{1}} \leq C\|Y \psi\|^{\frac{2}{3}}\|\psi\|_{L^{\infty}}^{\frac{1}{3}} \leq C\|Y \psi\|^{\frac{2}{3}}\left\|\partial_{Y} \psi\right\|^{\frac{1}{6}}\|\psi\|^{\frac{1}{6}}$,

$$
\begin{aligned}
\|\psi\|_{L^{1}} & \leq C\left(\frac{1}{\varepsilon^{\frac{2}{3}}}\|Y f\|+\frac{1}{\varepsilon^{\frac{1}{3}}}\|f\|\right)^{\frac{2}{3}} \frac{1}{\varepsilon^{\frac{1}{6}}}\|f\|^{\frac{1}{6}} \frac{1}{\varepsilon^{\frac{1}{9}}}\|f\|^{\frac{1}{6}} \\
& \leq C\left(\frac{1}{\varepsilon^{\frac{2}{3}}}\|Y f\|+\frac{1}{\varepsilon^{\frac{1}{3}}}\|f\|\right)^{\frac{2}{3}} \frac{1}{\varepsilon^{\frac{5}{18}}}\|f\|^{\frac{1}{3}} .
\end{aligned}
$$

This implies (6.16). We observe that from the integration by parts,

$$
\begin{aligned}
\int_{0}^{\infty} U_{s} \psi d Y & =-i \varepsilon \int_{0}^{\infty}\left(\partial_{Y}^{2}-\alpha^{2}\right) \psi d Y+\int_{0}^{\infty} \partial_{Y} f d Y \\
& =i \varepsilon \alpha^{2} \int_{0}^{\infty} \psi d Y .
\end{aligned}
$$

Then (6.17) follows from the $L^{1}$ estimate of $\psi$ in (6.16). Finally, we observe that $\sigma\left[U_{s} \psi\right](Y)=$ $\int_{Y}^{\infty} U_{s} \psi d Y_{1}$ satisfies

$$
\sigma\left[U_{s} \psi\right]=i \varepsilon \partial_{Y} \psi+i \varepsilon \alpha^{2} \int_{Y}^{\infty} \psi d Y_{1}-f=i \varepsilon \partial_{Y} \psi+i \varepsilon \alpha^{2} \sigma[\psi]-f .
$$

Thus we have

$$
\left\|(1+Y) \sigma\left[U_{s} \psi\right]\right\| \leq \varepsilon\left\|(1+Y) \partial_{Y} \psi\right\|+\varepsilon \alpha^{2}\|(1+Y) \sigma[\psi]\|+\|(1+Y) f\|,
$$

and then, it follows from Proposition 2.2 (1) that

$$
\left\|(1+Y) \sigma\left[U_{s} \psi\right]\right\| \leq \varepsilon\left\|(1+Y) \partial_{Y} \psi\right\|+C \varepsilon \alpha^{2}\|Y(1+Y) \psi\|+\|(1+Y) f\| .
$$

Hence, for $0 \leq \alpha \leq 1,(6.13),(6.22),(6.22)$, and (6.24) yield $\left\|(1+Y) \sigma\left[U_{s} \psi\right]\right\| \leq C\left\|(1+Y)^{2} f\right\|$, as desired. The proof is complete. 


\section{Orr-Sommerfeld equation}

Set $O S[\phi]=\operatorname{Ray}[\phi]+i \varepsilon\left(\partial_{Y}^{2}-\alpha^{2}\right)^{2} \phi$. This aim of this section is to solve the Orr-Sommerfeld equation

$$
\left\{\begin{array}{l}
O S[\phi]=f, \quad Y>0, \\
\left.\phi\right|_{Y=0}=\left.\partial_{Y} \phi\right|_{Y=0}=0 .
\end{array}\right.
$$

We assume that $\tilde{1} \sqrt{\nu} \leq \alpha \ll \varepsilon^{-\frac{1}{3}}$ with $\varepsilon=1 / \tilde{n}$, which means $\tilde{1} \leq \tilde{n} \ll \nu^{-\frac{3}{4}}$. To simplify the statement we focus on the case when $f$ decays fast enough, though this condition can be relaxed to some extent.

Theorem 7.1. There exist positive numbers $\delta_{0}, \varepsilon_{0}$ such that if $0<\varepsilon \leq \varepsilon_{0}$ and $0<\varepsilon^{\frac{1}{3}} \alpha \leq \delta_{0}$ then for any $f \in L^{2}\left(\mathbb{R}_{+}^{2}\right)$ with $(1+Y)^{2} f \in L^{2}\left(\mathbb{R}_{+}\right)$there exists a unique solution $\phi \in$ $H^{4}\left(\mathbb{R}_{+}\right) \cap H_{0}^{2}\left(\mathbb{R}_{+}\right)$to (7.1) satisfying

(i) when $\alpha \geq 1$ :

$$
\begin{aligned}
\left\|\partial_{Y} \phi\right\|+\alpha\|\phi\| & \leq C\|(1+Y) f\|, \\
\left\|\left(\partial_{Y}^{2}-\alpha^{2}\right) \phi\right\| & \leq \frac{C}{\varepsilon^{\frac{1}{3}}}\|(1+Y) f\|,
\end{aligned}
$$

(ii) when $0<\alpha \leq 1$ :

$$
\begin{aligned}
\left\|\partial_{Y} \phi\right\|+\alpha\|\phi\| & \leq \frac{\alpha+\varepsilon^{\frac{1}{6}}}{\alpha+\varepsilon^{\frac{1}{3}}}\left(\left\|(1+Y)^{2} f\right\|+\frac{1}{\alpha}\left|\int_{0}^{\infty} f d Y\right|\right), \\
\left\|\partial_{Y} \phi\right\|_{L^{\infty}} & \leq \frac{C}{\varepsilon^{\frac{1}{6}}}\left(\left\|(1+Y)^{2} f\right\|+\frac{1}{\alpha}\left|\int_{0}^{\infty} f d Y\right|\right), \\
\left\|\left(\partial_{Y}^{2}-\alpha^{2}\right) \phi\right\| & \leq \frac{C}{\varepsilon^{\frac{1}{3}}}\left(\left\|(1+Y)^{2} f\right\|+\frac{1}{\alpha}\left|\int_{0}^{\infty} f d Y\right|\right) .
\end{aligned}
$$

Remark 7.2. (i) When $\alpha$ is small, the singular factor $\alpha^{-1}$ in front of $\left|\int_{0}^{\infty} f d Y\right|$ is due to the Rayleigh equation and Proposition 5.1. It can not be dropped in general.

(ii) Note that the $L^{\infty}$ estimate (7.5) is not a consequence of the interpolation between (7.4) and (7.6). The loss of the factor $\varepsilon^{-\frac{1}{6}}$ in (7.4) appearing in the case $0<\alpha \ll 1$ comes from the slow mode of the boundary corrector. However, we can recover the estimates of $\left\|\partial_{Y} \phi\right\|_{L^{\infty}}$ and $\left\|\left(\partial_{Y}^{2}-\alpha^{2}\right) \phi\right\|$ as in (7.5) and (7.6), that are considered to be optimal in view of scaling.

\subsection{Rayleigh-Airy iteration}

In this subsection we consider the modified Orr-Sommerfeld equation

$$
\left\{\begin{array}{l}
O S[\Phi]=f, \quad Y>0, \\
\left.\Phi\right|_{Y=0}=\left.\partial_{Y} H_{\alpha} \Phi\right|_{Y=0}=0
\end{array}\right.
$$

where the self-adjoint operator $H_{\alpha}=\partial_{Y}^{2}-\alpha^{2}$ is realized in $L^{2}\left(\mathbb{R}_{+}\right)$with the domain $H^{2}\left(\mathbb{R}_{+}\right) \cap$ $H_{0}^{1}\left(\mathbb{R}_{+}\right)$. That is, the original boundary condition on $\left.\partial_{Y} \Phi\right|_{Y=0}=0$ is replaced by $\left.\partial_{Y} H_{\alpha} \Phi\right|_{Y=0}=$ 0 . To be rigorous our aim is to construct the solution $\Phi \in H^{2}\left(\mathbb{R}_{+}\right) \cap H_{0}^{1}\left(\mathbb{R}_{+}\right)$to the problem in the weak formulation

$$
\left\langle U_{s} H_{\alpha} \Phi-U_{s}^{\prime \prime} \Phi, q\right\rangle+i \varepsilon\left\langle H_{\alpha} \Phi,\left(\partial_{Y}^{2}-\alpha^{2}\right) q\right\rangle=\langle f, q\rangle, \quad q \in H^{2}\left(\mathbb{R}_{+}\right) \text {with } \partial_{Y} q(0)=0 .
$$


To state the main result of this subsection it is convenient to introduce the functions $\varphi_{1}, \psi_{0} \in$ $H^{2}\left(\mathbb{R}_{+}\right) \cap H_{0}^{1}\left(\mathbb{R}_{+}\right)$, which are respectively the solutions to

$$
\operatorname{Ray}\left[\varphi_{1}\right]=f, \quad \operatorname{Airy}\left[\psi_{0}\right]=-i \varepsilon \frac{f}{U_{s}}, \quad \frac{f}{U_{s}} \in L^{2}\left(\mathbb{R}_{+}\right) .
$$

Proposition 7.3. There exists a positive number $\varepsilon_{1}$ such that the following statement holds for any $0<\varepsilon \leq \varepsilon_{1}$ and $\alpha>0$. Let $f / U_{s} \in L^{2}\left(\mathbb{R}_{+}\right)$. Then there exists a solution $\Phi \in$ $H^{4}\left(\mathbb{R}_{+}\right) \cap H_{0}^{1}\left(\mathbb{R}_{+}\right)$to (7.7) satisfying the following estimates.

(i) when $\alpha \geq 1$,

$$
\begin{gathered}
\left\|\partial_{Y}\left(\Phi-\varphi_{1}-\psi_{0}\right)\right\| \leq C \varepsilon^{\frac{1}{3}}\left\|\partial_{Y} \varphi_{1}\right\|+\frac{C}{\varepsilon^{\frac{1}{3}}}\left(\left\|\psi_{0}\right\|+\frac{1}{\varepsilon^{\frac{1}{3}}}\left\|U_{s} \psi_{0}\right\|\right) \\
\alpha\left\|\Phi-\varphi_{1}-\psi_{0}\right\| \leq C \varepsilon^{\frac{1}{3}}\left(1+\alpha \varepsilon^{\frac{1}{3}}\right)\left\|\partial_{Y} \varphi_{1}\right\|+C\left(\alpha \varepsilon^{\frac{1}{3}}+\frac{1}{\varepsilon^{\frac{1}{3}}}\right)\left(\left\|\psi_{0}\right\|+\frac{1}{\varepsilon^{\frac{1}{3}}}\left\|U_{s} \psi_{0}\right\|\right) \\
\left\|\left(\partial_{Y}^{2}-\alpha^{2}\right)\left(\Phi-\varphi_{1}-\psi_{0}\right)\right\| \leq C\left\|\partial_{Y} \varphi_{1}\right\|+\frac{C}{\varepsilon^{\frac{2}{3}}}\left\|\psi_{0}\right\| .
\end{gathered}
$$

(ii) when $0<\alpha \leq 1$,

$$
\begin{aligned}
\left\|\partial_{Y}\left(\Phi-\varphi_{1}-\psi_{0}\right)\right\| & \leq C \varepsilon^{\frac{1}{3}}\left\|\partial_{Y} \varphi_{1}\right\|+\frac{C}{\varepsilon^{\frac{1}{3}}}\left(\left\|\psi_{0}\right\|+\frac{1}{\varepsilon^{\frac{1}{3}}}\left\|U_{s} \psi_{0}\right\|\right), \\
\alpha\left\|\Phi-\varphi_{1}-\psi_{0}\right\| & \leq C \alpha \varepsilon^{\frac{1}{3}}\left\|U_{s}^{\prime \prime} \varphi_{1}\right\|+C \alpha\left\|\psi_{0}\right\|, \\
\left\|\left(\partial_{Y}^{2}-\alpha^{2}\right)\left(\Phi-\varphi_{1}-\psi_{0}\right)\right\| & \leq C\left\|\partial_{Y} \varphi_{1}\right\|+\frac{C}{\varepsilon^{\frac{2}{3}}}\left\|\psi_{0}\right\| .
\end{aligned}
$$

Proof. We apply the iteration argument, called the Rayleigh-Airy iteration. Let $\varphi_{1} \in H^{2}\left(\mathbb{R}_{+}\right) \cap$ $H_{0}^{1}\left(\mathbb{R}_{+}\right)$be the solution to the Rayleigh equation (5.1). Then we have

$$
\left\langle U_{s} H_{\alpha} \varphi_{1}-U_{s}^{\prime \prime} \varphi_{1}, q\right\rangle+i \varepsilon\left\langle H_{\alpha} \varphi_{1},\left(\partial_{Y}^{2}-\alpha^{2}\right) q\right\rangle=\langle f, q\rangle+i \varepsilon\left\langle H_{\alpha} \varphi_{1},\left(\partial_{Y}^{2}-\alpha^{2}\right) q\right\rangle, \quad q \in H^{2}\left(\mathbb{R}_{+}\right) .
$$

Note that the identity (7.15) holds for any $q \in H^{2}\left(\mathbb{R}_{+}\right)$rather than $q \in H^{2}\left(\mathbb{R}_{+}\right)$with $\partial_{Y} q(0)=$ 0 . To correct the error term $i \varepsilon H_{\alpha}^{2} \varphi_{1}$, we observe the identity

$$
\begin{aligned}
H_{\alpha}[\varphi] & =\left(\left(\partial_{Y}^{2}-\alpha^{2}\right)-\frac{U_{s}^{\prime \prime}}{U_{s}}\right) \varphi+\frac{U_{s}^{\prime \prime}}{U_{s}} \varphi \\
& =\frac{1}{U_{s}} \operatorname{Ray}[\varphi]+\frac{U_{s}^{\prime \prime}}{U_{s}} \varphi .
\end{aligned}
$$

Hence we have from $\operatorname{Ray}\left[\varphi_{1}\right]=f$,

$$
\begin{gathered}
\left\langle U_{s} H_{\alpha} \varphi_{1}-U_{s}^{\prime \prime} \varphi_{1}, q\right\rangle+i \varepsilon\left\langle H_{\alpha} \varphi_{1},\left(\partial_{Y}^{2}-\alpha^{2}\right) q\right\rangle=\langle f, q\rangle+i \varepsilon\left\langle\frac{f}{U_{s}}+\frac{U_{s}^{\prime \prime}}{U_{s}} \varphi_{1},\left(\partial_{Y}^{2}-\alpha^{2}\right) q\right\rangle, \\
q \in H^{2}\left(\mathbb{R}_{+}\right) .
\end{gathered}
$$

Our next task is to recover $\left(\partial_{Y}^{2}-\alpha^{2}\right)$ regularity in the error term. To this end we observe the relation

$$
\left[U_{s}, H_{\alpha}\right] h-U_{s}^{\prime \prime} h=-2 \partial_{Y}\left(U_{s}^{\prime} h\right),
$$


and then formally we have

$$
O S[\varphi]=\left(\partial_{Y}^{2}-\alpha^{2}\right)\left(U_{s} \varphi+i \varepsilon\left(\partial_{Y}^{2}-\alpha^{2}\right)\right) \varphi-2 \partial_{Y}\left(U_{s}^{\prime} \varphi\right)=\left(\partial_{Y}^{2}-\alpha^{2}\right) \operatorname{Airy}[\varphi]-2 \partial_{Y}\left(U_{s}^{\prime} \varphi\right) .
$$

Thus we take $\psi_{1} \in H^{2}\left(\mathbb{R}_{+}\right) \cap H_{0}^{1}\left(\mathbb{R}_{+}\right)$as the solution to the Airy equation

$$
\operatorname{Airy}\left[\psi_{1}\right]=-i \varepsilon\left(\frac{f}{U_{s}}+\frac{U_{s}^{\prime \prime}}{U_{s}} \varphi_{1}\right)
$$

and then $\varphi_{1}+\psi_{1}$ satisfies

$$
\begin{aligned}
& \left\langle U_{s} H_{\alpha}\left(\varphi_{1}+\psi_{1}\right)-U_{s}^{\prime \prime}\left(\varphi_{1}+\psi_{1}\right), q\right\rangle+i \varepsilon\left\langle H_{\alpha}\left(\varphi_{1}+\psi_{1}\right),\left(\partial_{Y}^{2}-\alpha^{2}\right) q\right\rangle \\
& =\langle f, q\rangle+i \varepsilon\left\langle\frac{f}{U_{s}}+\frac{U_{s}^{\prime \prime}}{U_{s}} \varphi_{1},\left(\partial_{Y}^{2}-\alpha^{2}\right) q\right\rangle \\
& \quad+\left\langle H_{\alpha} U_{s} \psi_{1}, q\right\rangle+\left\langle-2 \partial_{Y}\left(U_{s}^{\prime} \psi_{1}\right), q\right\rangle+i \varepsilon\left\langle H_{\alpha} \psi_{1},\left(\partial_{Y}^{2}-\alpha^{2}\right) q\right\rangle \\
& =\langle f, q\rangle+i \varepsilon\left\langle\frac{f}{U_{s}}+\frac{U_{s}^{\prime \prime}}{U_{s}} \varphi_{1},\left(\partial_{Y}^{2}-\alpha^{2}\right) q\right\rangle \\
& \quad+\left\langle U_{s} \psi_{1},\left(\partial_{Y}^{2}-\alpha^{2}\right) q\right\rangle+i \varepsilon\left\langle H_{\alpha} \psi_{1},\left(\partial_{Y}^{2}-\alpha^{2}\right) q\right\rangle+\left\langle-2 \partial_{Y}\left(U_{s}^{\prime} \psi_{1}\right), q\right\rangle \\
& =\langle f, q\rangle+\left\langle-2 \partial_{Y}\left(U_{s}^{\prime} \psi_{1}\right), q\right\rangle, \quad q \in H^{2}\left(\mathbb{R}_{+}\right) .
\end{aligned}
$$

Note that, due to the fact that $\left.\partial_{Y}^{k}\left(U_{s} \psi_{1}\right)\right|_{Y=0}$ for $k=0,1$, the equality (7.17) is valid for $q \in H^{2}\left(\mathbb{R}_{+}\right)$rather than $q \in H^{2}\left(\mathbb{R}_{+}\right)$with $\partial_{Y} q(0)=0$. The new error term is $-2 \partial_{Y}\left(U_{s}^{\prime} \psi_{1}\right)$, but this is not compatible in solving the Rayleigh equation since $-2 \partial_{Y}\left(U_{s}^{\prime} \psi_{1}\right) / U_{s}$ does not belong to $L^{2}\left(\mathbb{R}_{+}\right)$in general. Hence, we next take $\tilde{\psi}_{1} \in \underset{\sim}{H^{2}}\left(\mathbb{R}_{+}\right)$as the solution to Airy $\left[\tilde{\psi}_{1}\right]=$ $2 \partial_{Y}\left(U_{s}^{\prime} \psi_{1}\right)$ under the Neumann boundary condition $\left.\partial_{Y} \tilde{\psi}_{1}\right|_{Y=0}=0$, and set $\varphi_{2}$ as the solution to the Rayleigh equation $\operatorname{Ray}\left[\varphi_{2}\right]=U_{s} \tilde{\psi}_{1}$ with $\left.\varphi_{2}\right|_{Y=0}=0$. Then $\varphi_{2} \in H^{2}\left(\mathbb{R}_{+}\right) \cap H_{0}^{1}\left(\mathbb{R}_{+}\right)$ and from the formal identity

we have

$$
\begin{aligned}
O S[\varphi] & =\left(U_{s}+i \varepsilon\left(\partial_{Y}^{2}-\alpha^{2}\right)\right)\left(\left(\partial_{Y}^{2}-\alpha^{2}\right)-\frac{U_{s}^{\prime \prime}}{U_{s}}\right) \varphi+i \varepsilon\left(\partial_{Y}^{2}-\alpha^{2}\right) \frac{U_{s}^{\prime \prime}}{U_{s}} \varphi \\
& =\operatorname{Airy}\left[\frac{1}{U_{s}} \operatorname{Ray}[\varphi]\right]+i \varepsilon\left(\partial_{Y}^{2}-\alpha^{2}\right) \frac{U_{s}^{\prime \prime}}{U_{s}} \varphi
\end{aligned}
$$

$$
O S\left[\varphi_{2}\right]=\operatorname{Airy}\left[\frac{1}{U_{s}} U_{s} \tilde{\psi}_{1}\right]+i \varepsilon\left(\partial_{Y}^{2}-\alpha^{2}\right) \frac{U_{s}^{\prime \prime}}{U_{s}} \varphi_{2}=2 \partial_{Y}\left(U_{s}^{\prime} \psi_{1}\right)+i \varepsilon\left(\partial_{Y}^{2}-\alpha^{2}\right) \frac{U_{s}^{\prime \prime}}{U_{s}} \varphi_{2} .
$$

This formal identity is rigorously justified in the weak formulation as follows. Let $q \in H^{2}\left(\mathbb{R}_{+}\right)$ with $\partial_{Y} q(0)=0$. Then $\varphi_{1}+\psi_{1}+\varphi_{2}$ solves

$$
\begin{aligned}
& \left\langle U_{s} H_{\alpha}\left(\varphi_{1}+\psi_{1}+\varphi_{2}\right)-U_{s}^{\prime \prime}\left(\varphi_{1}+\psi_{1}+\varphi_{2}\right), q\right\rangle+i \varepsilon\left\langle H_{\alpha}\left(\varphi_{1}+\psi_{1}+\varphi_{2}\right),\left(\partial_{Y}^{2}-\alpha^{2}\right) q\right\rangle \\
& =\langle f, q\rangle+\left\langle-2 \partial_{Y}\left(U_{s}^{\prime} \psi_{1}\right), q\right\rangle+\left\langle U_{s} \tilde{\psi}_{1}, q\right\rangle \\
& \quad+i \varepsilon\left\langle\frac{1}{U_{s}}\left(U_{s}\left(\partial_{Y}^{2}-\alpha^{2}\right)-U_{s}^{\prime \prime}\right) \varphi_{2},\left(\partial_{Y}^{2}-\alpha^{2}\right) q\right\rangle+i \varepsilon\left\langle\frac{U_{s}^{\prime \prime}}{U_{s}} \varphi_{2},\left(\partial_{Y}^{2}-\alpha^{2}\right) q\right\rangle \\
& =\langle f, q\rangle+\left\langle-2 \partial_{Y}\left(U_{s}^{\prime} \psi_{1}\right), q\right\rangle+\left\langle U_{s} \tilde{\psi}_{1}, q\right\rangle+i \varepsilon\left\langle\frac{1}{U_{s}} U_{s} \tilde{\psi}_{1},\left(\partial_{Y}^{2}-\alpha^{2}\right) q\right\rangle+i \varepsilon\left\langle\frac{U_{s}^{\prime \prime}}{U_{s}} \varphi_{2},\left(\partial_{Y}^{2}-\alpha^{2}\right) q\right\rangle \\
& =\langle f, q\rangle+\left\langle-2 \partial_{Y}\left(U_{s}^{\prime} \psi_{1}\right), q\right\rangle+\left\langle\operatorname{Airy}\left[\tilde{\psi}_{1}\right], q\right\rangle+i \varepsilon\left\langle\frac{U_{s}^{\prime \prime}}{U_{s}} \varphi_{2},\left(\partial_{Y}^{2}-\alpha^{2}\right) q\right\rangle \\
& \quad \quad\left(\operatorname{since} q \in H^{2}\left(\mathbb{R}_{+}\right) \text {with } \partial_{Y} q(0)=0\right) \\
& =\langle f, q\rangle+\left\langle-2 \partial_{Y}\left(U_{s}^{\prime} \psi_{1}\right), q\right\rangle+\left\langle 2 \partial_{Y}\left(U_{s}^{\prime} \psi_{1}\right), q\right\rangle+\left\langle i \varepsilon \frac{U_{s}^{\prime \prime}}{U_{s}} \varphi_{2},\left(\partial_{Y}^{2}-\alpha^{2}\right) q\right\rangle \\
& =\langle f, q\rangle+i \varepsilon\left\langle\frac{U_{s}^{\prime \prime}}{U_{s}} \varphi_{2},\left(\partial_{Y}^{2}-\alpha^{2}\right) q\right\rangle, \quad q \in H^{2}\left(\mathbb{R}_{+}\right) \text {with } \partial_{Y} q(0)=0 .
\end{aligned}
$$


The error term $i \varepsilon\left(\partial_{Y}^{2}-\alpha^{2}\right) \frac{U_{s}^{\prime \prime}}{U_{s}} \varphi_{2}$ (in the weak form) is then handled by solving the Airy equation with the source $-i \varepsilon \frac{U_{s}^{\prime \prime}}{U_{s}} \varphi_{2}$ by using the formal relation (7.16), which creates the next error terms. We iterate this process, namely, for $k \geq 1$ we set

(i) $\varphi_{k+1} \in H^{2}\left(\mathbb{R}_{+}\right) \cap H_{0}^{1}\left(\mathbb{R}_{+}\right)$as the solution to the Rayleigh equation $\operatorname{Ray}\left[\varphi_{k+1}\right]=U_{s} \tilde{\psi}_{k}$,

(ii) $\psi_{k+1} \in H^{2}\left(\mathbb{R}_{+}\right) \cap H_{0}^{1}\left(\mathbb{R}_{+}\right)$as the solution to the Airy equation Airy $\left[\psi_{k+1}\right]=-i \varepsilon \frac{U_{s}^{\prime \prime}}{U_{s}} \varphi_{k+1}$,

(iii) $\tilde{\psi}_{k+1} \in H^{2}\left(\mathbb{R}_{+}\right)$as the solution to $\operatorname{Airy}\left[\tilde{\psi}_{k+1}\right]=2 \partial_{Y}\left(U_{s}^{\prime} \psi_{k+1}\right)$ under the Neumann boundary condition $\left.\partial_{Y} \tilde{\psi}_{k+1}\right|_{Y=0}=0$.

Then $\Phi_{m}=\varphi_{1}+\psi_{1}+\sum_{k=2}^{m} \varphi_{k}+\sum_{k=2}^{m} \psi_{k}, m \geq 2$, solves

$$
\begin{aligned}
\left\langle U_{s} H_{\alpha} \Phi_{m}-U_{s}^{\prime \prime} \Phi_{m}, q\right\rangle+i \varepsilon\left\langle H_{\alpha} \Phi_{m},\left(\partial_{Y}^{2}-\alpha^{2}\right) q\right\rangle=\langle f, q\rangle+\left\langle-2 \partial_{Y}\left(U_{s}^{\prime} \psi_{m}\right), q\right\rangle, & \\
& q \in H^{2}\left(\mathbb{R}_{+}\right) \text {with } \partial_{Y} q(0)=0 .
\end{aligned}
$$

Our next aim is to show that $\Phi_{m}$ converges in $H^{2}\left(\mathbb{R}_{+}\right) \cap H_{0}^{1}\left(\mathbb{R}_{+}\right)$. Then the limit $\Phi=\lim _{m \rightarrow \infty} \Phi_{m}$ solves the weak formulation (7.8), and then the regularity $H_{\alpha} \Phi \in H^{2}\left(\mathbb{R}_{+}\right)$is recovered from the weak formulation by regarding the term $U_{s} H_{\alpha} \Phi-U_{s}^{\prime \prime} \Phi \in L^{2}\left(\mathbb{R}_{+}\right)$as the source term; indeed, (7.8) implies that the limit $H_{\alpha} \Phi$ is the very weak solution to the Poisson equation $i \varepsilon\left(\partial_{Y}^{2}-\alpha^{2}\right) H_{\alpha} \Phi=-U_{s} H_{\alpha} \Phi+U_{s}^{\prime \prime} \Phi+f \in L^{2}\left(\mathbb{R}_{+}\right)$subject to the zero Neumann boundary condition.

Remark 7.4. The additional boundary condition $\left.\partial_{Y} H_{\alpha} \Phi\right|_{h=0}=0$, that is derived and understood in a weak sense through the variational formulation (7.8), can also be recovered at a formal level by manipulating the strong formulation of the equations. More precisely, one can derive the identity $\left.\partial_{Y} H_{\alpha}\left(\varphi_{k}+\psi_{k}\right)\right|_{Y=0}=0$ for all $k \geq 1$ as follows. For $k \geq 2$, we differentiate the Airy equation satisfied by $\psi_{k}$ and take its trace to find

$$
\left.i \varepsilon \partial_{Y} H_{\alpha} \psi_{k}\right|_{Y=0}=-\left.i \varepsilon \partial_{Y}\left(\frac{U_{s}^{\prime \prime}}{U_{s}} \varphi_{k}\right)\right|_{Y=0} .
$$

We have used here that $\left.\partial_{Y}\left(U_{s} \psi_{k}\right)\right|_{Y=0}=0$ due to the Dirichlet boundary condition on $\psi_{k}$. Similarly, we divide the Rayleigh equation satisfied by $\varphi_{\tilde{\sim}}$ by $U_{s}$, differentiate it and take its trace to find (using the Neumann condition satisfied by $\tilde{\psi}_{k-1}$ ):

$$
\left.\partial_{Y} H_{\alpha} \varphi_{k}\right|_{Y=0}=\left.\partial_{Y}\left(\frac{U_{s}^{\prime \prime}}{U_{s}} \varphi_{k}\right)\right|_{Y=0} .
$$

The identity $\left.\partial_{Y} H_{\alpha}\left(\varphi_{k}+\psi_{k}\right)\right|_{Y=0}=0$ follows for all $k \geq 2$. The same result holds for $k=1$ taking into account the additional source term $f$.

To show the convergence we divide into two cases $\alpha \geq 1$ and $0<\alpha \leq 1$.

(1) The case $\alpha \geq 1$ : We have from (i) above and (5.2), for $k \geq 1$

$$
\left\|\partial_{Y} \varphi_{k+1}\right\|+\alpha\left\|\varphi_{k+1}\right\| \leq C\left\|Y \tilde{\psi}_{k}\right\|,
$$

while from (5.3),

$$
\left\|\left(\partial_{Y}^{2}-\alpha^{2}\right) \varphi_{k+1}\right\| \leq C\left\|Y \tilde{\psi}_{k}\right\|+\left\|\tilde{\psi}_{k}\right\| .
$$

Here $C$ is independent of $\alpha \geq 1$. Then Proposition 6.3 implies, for $k \geq 1$,

$$
\begin{aligned}
\left\|Y \tilde{\psi}_{k}\right\| & \leq \frac{C}{\varepsilon^{\frac{2}{3}}}\left\|Y U_{s}^{\prime} \psi_{k}\right\|+\frac{C}{\varepsilon^{\frac{1}{3}}}\left\|U_{s}^{\prime} \psi_{k}\right\| \leq \frac{C}{\varepsilon^{\frac{2}{3}}}\left\|U_{s} \psi_{k}\right\|+\frac{C}{\varepsilon^{\frac{1}{3}}}\left\|\psi_{k}\right\|, \\
\left\|\tilde{\psi}_{k}\right\| & \leq \frac{C}{\varepsilon^{\frac{2}{3}}}\left\|U_{s}^{\prime} \psi_{k}\right\| \leq \frac{C}{\varepsilon^{\frac{2}{3}}}\left\|\psi_{k}\right\| .
\end{aligned}
$$


Here $C$ is independent of $\alpha$. This gives

$$
\left\|\partial_{Y} \varphi_{k+1}\right\|+\alpha\left\|\varphi_{k+1}\right\| \leq \frac{C}{\varepsilon^{\frac{2}{3}}}\left\|U_{s} \psi_{k}\right\|+\frac{C}{\varepsilon^{\frac{1}{3}}}\left\|\psi_{k}\right\|, \quad\left\|\left(\partial_{Y}^{2}-\alpha^{2}\right) \varphi_{k+1}\right\| \leq \frac{C}{\varepsilon^{\frac{2}{3}}}\left\|\psi_{k}\right\| .
$$

Next Proposition 6.1 shows, for $k \geq 2$,

$$
\left\|U_{s} \psi_{k}\right\|+\varepsilon^{\frac{1}{3}}\left\|\psi_{k}\right\|+\varepsilon^{\frac{2}{3}}\left\|\partial_{Y} \psi_{k}\right\|+\varepsilon\left\|\left(\partial_{Y}^{2}-\alpha^{2}\right) \psi_{k}\right\| \leq C \varepsilon\left\|\frac{U_{s}^{\prime \prime}}{U_{s}} \varphi_{k}\right\| \leq C \varepsilon\left\|\partial_{Y} \varphi_{k}\right\| .
$$

Hence (7.22) and (7.23) yield

$$
\left\|\partial_{Y} \varphi_{k+1}\right\|+\alpha\left\|\varphi_{k+1}\right\| \leq C \varepsilon^{\frac{1}{3}}\left\|\partial_{Y} \varphi_{k}\right\|,
$$

and thus, $\sum_{k=2}^{\infty} \varphi_{k}$ converges in $H^{1}\left(\mathbb{R}_{+}\right)$if $\varepsilon>0$ is small enough and satisfies

$$
\sum_{k=2}^{\infty}\left\|\partial_{Y} \varphi_{k}\right\|+\alpha \sum_{k=2}^{\infty}\left\|\varphi_{k}\right\| \leq C\left\|\partial_{Y} \varphi_{2}\right\|+\alpha\left\|\varphi_{2}\right\| \leq \frac{C}{\varepsilon^{\frac{2}{3}}}\left\|U_{s} \psi_{1}\right\|+\frac{C}{\varepsilon^{\frac{1}{3}}}\left\|\psi_{1}\right\| .
$$

Here we have used (7.22) in the last line. Then (7.22) and (7.23) show that $\sum_{k=2}^{\infty} \varphi_{k}$ converges in $H^{2}\left(\mathbb{R}_{+}\right)$and satisfies

$$
\sum_{k=2}^{\infty}\left\|\left(\partial_{Y}^{2}-\alpha^{2}\right) \varphi_{k}\right\| \leq C\left\|\partial_{Y} \varphi_{2}\right\|+\frac{C}{\varepsilon^{\frac{2}{3}}}\left\|\psi_{1}\right\| \leq \frac{C}{\varepsilon^{\frac{2}{3}}}\left\|\psi_{1}\right\| .
$$

As for the convergence and the estimate of $\sum_{k=2}^{\infty} \psi_{k}$, we have from (7.23) and (7.25),

$$
\sum_{k=2}^{\infty}\left\|\partial_{Y} \psi_{k}\right\| \leq C \varepsilon^{\frac{1}{3}} \sum_{k=2}^{\infty}\left\|\partial_{Y} \varphi_{k}\right\| \leq \frac{C}{\varepsilon^{\frac{1}{3}}}\left\|U_{s} \psi_{1}\right\|+C\left\|\psi_{1}\right\|,
$$

and similarly,

$$
\begin{aligned}
\alpha \sum_{k=2}^{\infty}\left\|\psi_{k}\right\| & \leq C \alpha \varepsilon^{\frac{2}{3}} \sum_{k=2}^{\infty}\left\|\partial_{Y} \varphi_{k}\right\| \leq C \alpha\left(\left\|U_{s} \psi_{1}\right\|+\varepsilon^{\frac{1}{3}}\left\|\psi_{1}\right\|\right), \\
\sum_{k=2}^{\infty}\left\|\left(\partial_{Y}^{2}-\alpha^{2}\right) \psi_{k}\right\| & \leq C \sum_{k=2}^{\infty}\left\|\partial_{Y} \varphi_{k}\right\| \leq \frac{C}{\varepsilon^{\frac{2}{3}}}\left\|U_{s} \psi_{1}\right\|+\frac{C}{\varepsilon^{\frac{1}{3}}}\left\|\psi_{1}\right\|
\end{aligned}
$$

Let us recall that $\psi_{1}$ is decomposed as $\psi_{1}=\psi_{0}+\psi_{1,1}$, where $\psi_{0}, \psi_{1,1} \in H^{2}\left(\mathbb{R}_{+}\right) \cap H_{0}^{1}\left(\mathbb{R}_{+}\right)$ are respectively the solutions to

$$
\operatorname{Airy}\left[\psi_{0}\right]=-i \varepsilon \frac{f}{U_{s}}, \quad \operatorname{Airy}\left[\psi_{1,1}\right]=-i \varepsilon \frac{U_{s}^{\prime \prime}}{U_{s}} \varphi_{1} .
$$

Then $\psi_{1,1}$ satisfies in virtue of (6.2) in Proposition 6.1,

$$
\left\|U_{s} \psi_{1,1}\right\|+\varepsilon^{\frac{1}{3}}\left\|\psi_{1,1}\right\|+\varepsilon^{\frac{2}{3}}\left\|\partial_{Y} \psi_{1,1}\right\|+\varepsilon\left\|\left(\partial_{Y}^{2}-\alpha^{2}\right) \psi_{1,1}\right\| \leq C \varepsilon\left\|\frac{U_{s}^{\prime \prime}}{U_{s}} \varphi_{1}\right\| \leq C \varepsilon\left\|\partial_{Y} \varphi_{1}\right\|,
$$

or we also have from (6.4) and (6.5),

$$
\varepsilon^{\frac{1}{3}}\left\|U_{s} \psi_{1,1}\right\|+\varepsilon^{\frac{2}{3}}\left\|\psi_{1,1}\right\|+\varepsilon\left\|\partial_{Y} \psi_{1,1}\right\| \leq C \varepsilon\left\|U_{s}^{\prime \prime} \varphi_{1}\right\| .
$$


Note that (7.30) and (7.31) are valid for all $\alpha>0$. Collecting (7.25), (7.26), (7.27), (7.28), (7.29), and (7.30), we obtain

$$
\begin{aligned}
\left\|\partial_{Y}\left(\Phi-\varphi_{1}-\psi_{0}\right)\right\| & \leq C \varepsilon^{\frac{1}{3}}\left\|\partial_{Y} \varphi_{1}\right\|+\frac{C}{\varepsilon^{\frac{1}{3}}}\left\|\psi_{0}\right\|+\frac{C}{\varepsilon^{\frac{2}{3}}}\left\|U_{s} \psi_{0}\right\|, \\
\alpha\left\|\Phi-\varphi_{1}-\psi_{0}\right\| & \leq C \varepsilon^{\frac{1}{3}}\left(1+\alpha \varepsilon^{\frac{1}{3}}\right)\left\|\partial_{Y} \varphi_{1}\right\|+C\left(\alpha \varepsilon^{\frac{1}{3}}+\frac{1}{\varepsilon^{\frac{1}{3}}}\right)\left(\left\|\psi_{0}\right\|+\frac{1}{\varepsilon^{\frac{1}{3}}}\left\|U_{s} \psi_{0}\right\|\right), \\
\left\|\left(\partial_{Y}^{2}-\alpha^{2}\right)\left(\Phi-\varphi_{1}-\psi_{0}\right)\right\| & \leq C\left\|\partial_{Y} \varphi_{1}\right\|+\frac{C}{\varepsilon^{\frac{2}{3}}}\left\|\psi_{0}\right\| .
\end{aligned}
$$

The proof for the case $\alpha \geq 1$ is complete.

(2) The case $0<\alpha \leq 1$ : We first observe from Proposition 6.3 that

$$
\begin{aligned}
\left\|(1+Y)^{2} U_{s} \tilde{\psi}_{k}\right\| \leq & C\left\|U_{s} \tilde{\psi}_{k}\right\|+C\left\|Y^{2} U_{s} \tilde{\psi}_{k}\right\| \\
\leq & \frac{C}{\varepsilon^{\frac{1}{3}}}\left\|U_{s}^{\prime} \psi_{k}\right\|+\frac{C}{\varepsilon^{\frac{2}{3}}}\left\|U_{s} U_{s}^{\prime} \psi_{k}\right\| \\
& +\frac{C}{\varepsilon^{\frac{2}{3}}}\left\|U_{s} Y^{2} U_{s}^{\prime} \psi_{k}\right\|+\frac{C}{\varepsilon^{\frac{1}{3}}}\left\|U_{s} Y U_{s}^{\prime} \psi_{k}\right\|+C\left\|Y U_{s}^{\prime} \psi_{k}\right\|+C \varepsilon^{\frac{1}{3}}\left\|U_{s}^{\prime} \psi_{k}\right\| \\
\leq & \frac{C}{\varepsilon^{\frac{1}{3}}}\left(\left\|\psi_{k}\right\|+\frac{1}{\varepsilon^{\frac{1}{3}}}\left\|U_{s} \psi_{k}\right\|\right),
\end{aligned}
$$

and

$$
\left|\int_{0}^{\infty} U_{s} \tilde{\psi}_{k} d Y\right| \leq C \alpha^{2}\left(\varepsilon^{\frac{1}{6}}\left\|Y U_{s}^{\prime} \psi_{k}\right\|+\varepsilon^{\frac{1}{2}}\left\|U_{s}^{\prime} \psi_{k}\right\|\right) .
$$

Thus Proposition 5.1 and Proposition 2.2 (1) yield, for $k \geq 1$,

$$
\begin{aligned}
\left\|\partial_{Y} \varphi_{k+1}\right\| & \leq C\left\|(1+Y)^{2} U_{s} \tilde{\psi}_{k}\right\|+\frac{C}{\alpha}\left|\int_{0}^{\infty} U_{s} \tilde{\psi}_{k} d Y\right| \\
& \leq \frac{C}{\varepsilon^{\frac{1}{3}}}\left(\left\|\psi_{k}\right\|+\frac{1}{\varepsilon^{\frac{1}{3}}}\left\|U_{s} \psi_{k}\right\|\right) .
\end{aligned}
$$

Then from (7.23), which is valid also in the case $0<\alpha \leq 1$ with $k \geq 2$, we have for $k \geq 2$,

$$
\left\|\partial_{Y} \varphi_{k+1}\right\| \leq C \varepsilon^{\frac{1}{3}}\left\|\frac{U_{s}^{\prime \prime}}{U_{s}} \varphi_{k}\right\| \leq C \varepsilon^{\frac{1}{3}}\left\|\partial_{Y} \varphi_{k}\right\| .
$$

Similarly, for all $k \geq 1$,

$$
\begin{aligned}
\left\|\left(\partial_{Y}^{2}-\alpha^{2}\right) \varphi_{k+1}\right\| & \leq C\left\|(1+Y)^{2} U_{s} \tilde{\psi}_{k}\right\|+\left\|\tilde{\psi}_{k}\right\|+\frac{C}{\alpha}\left|\int_{0}^{\infty} U_{s} \tilde{\psi}_{k} d Y\right| \\
& \leq \frac{C}{\varepsilon^{\frac{1}{3}}}\left\|\psi_{k}\right\|+\frac{C}{\varepsilon^{\frac{2}{3}}}\left\|U_{s} \psi_{k}\right\|+\frac{C}{\varepsilon^{\frac{2}{3}}}\left\|\psi_{k}\right\| \\
& \leq \frac{C}{\varepsilon^{\frac{2}{3}}}\left\|\psi_{k}\right\|,
\end{aligned}
$$

which implies when $k \geq 2$ :

$$
\left\|\left(\partial_{Y}^{2}-\alpha^{2}\right) \varphi_{k+1}\right\| \leq C\left\|\partial_{Y} \varphi_{k}\right\| .
$$

Next we observe from (6.18) that, for $\sigma\left[U_{s} \tilde{\psi}_{k}\right](Y)=\int_{Y}^{\infty} U_{s} \tilde{\psi}_{k} d Y_{1}$,

$$
\left\|(1+Y) \sigma\left[U_{s} \tilde{\psi}_{k}\right]\right\| \leq C\left\|(1+Y)^{2} U_{s}^{\prime} \psi_{k}\right\| \leq C\left\|\psi_{k}\right\| .
$$


Thus we have again from Proposition 5.1, for all $k \geq 1$,

$$
\begin{aligned}
\alpha\left\|\varphi_{k+1}\right\| & \leq C \alpha\left\|(1+Y) \sigma\left[U_{s} \tilde{\psi}_{k}\right]\right\|+\frac{C}{\alpha^{\frac{1}{2}}}\left|\int_{0}^{\infty} U_{s} \tilde{\psi}_{k} d Y\right| \\
& \leq C \alpha\left\|\psi_{k}\right\|
\end{aligned}
$$

and for $k \geq 2$, by (7.23),

$$
\alpha\left\|\varphi_{k+1}\right\| \leq C \alpha \epsilon^{\frac{2}{3}}\left\|\partial_{Y} \varphi_{k}\right\|
$$

This ensure the convergence of $\sum_{k=2}^{\infty} \varphi_{k}$ in $H^{2}\left(\mathbb{R}_{+}\right)$when $\varepsilon$ is small enough, and we have

$$
\begin{gathered}
\sum_{k=2}^{\infty}\left\|\partial_{Y} \varphi_{k}\right\| \leq C\left\|\partial_{Y} \varphi_{2}\right\| \leq \frac{C}{\varepsilon^{\frac{1}{3}}}\left(\left\|\psi_{1}\right\|+\frac{1}{\varepsilon^{\frac{1}{3}}}\left\|U_{s} \psi_{1}\right\|\right), \\
\sum_{k=2}^{\infty} \alpha\left\|\varphi_{k}\right\| \leq \alpha\left\|\varphi_{2}\right\|+C \alpha \varepsilon^{\frac{2}{3}}\left\|\partial_{Y} \varphi_{2}\right\| \leq C \alpha\left\|\psi_{1}\right\|, \\
\sum_{k=2}^{\infty}\left\|\left(\partial_{Y}^{2}-\alpha^{2}\right) \varphi_{k}\right\| \leq\left\|\left(\partial_{Y}^{2}-\alpha^{2}\right) \varphi_{2}\right\|+C \sum_{k=2}^{\infty}\left\|\partial_{Y} \varphi_{k}\right\| \leq \frac{C}{\varepsilon^{\frac{2}{3}}}\left\|\psi_{1}\right\| .
\end{gathered}
$$

Then, by applying Proposition $6.1, \sum_{k=2}^{\infty} \psi_{k}$ converges in $H^{2}\left(\mathbb{R}_{+}\right)$as in the case $\alpha \geq 1$, and we have

$$
\begin{array}{r}
\sum_{k=2}^{\infty}\left\|\partial_{Y} \psi_{k}\right\| \leq C \varepsilon^{\frac{1}{3}} \sum_{k=2}^{\infty}\left\|\partial_{Y} \varphi_{k}\right\| \leq C\left(\left\|\psi_{1}\right\|+\frac{1}{\varepsilon^{\frac{1}{3}}}\left\|U_{s} \psi_{1}\right\|\right), \\
\sum_{k=2}^{\infty} \alpha\left\|\psi_{k}\right\| \leq C \alpha \varepsilon^{\frac{2}{3}} \sum_{k=2}^{\infty}\left\|\partial_{Y} \varphi_{k}\right\| \leq C \alpha \varepsilon^{\frac{1}{3}}\left(\left\|\psi_{1}\right\|+\frac{1}{\varepsilon^{\frac{1}{3}}}\left\|U_{s} \psi_{1}\right\|\right), \\
\sum_{k=2}^{\infty}\left\|\left(\partial_{Y}^{2}-\alpha^{2}\right) \psi_{k}\right\| \leq C \sum_{k=2}^{\infty}\left\|\partial_{Y} \varphi_{k}\right\| \leq \frac{C}{\varepsilon^{\frac{1}{3}}}\left(\left\|\psi_{1}\right\|+\frac{C}{\varepsilon^{\frac{1}{3}}}\left\|U_{s} \psi_{1}\right\|\right) .
\end{array}
$$

Recall that $\psi_{1}$ is decomposed as $\psi_{1}=\psi_{0}+\psi_{1,1}$ as in the case $\alpha \geq 1$. Hence, from (7.37), (7.38), and the estimates for $\psi_{1,1}$ in (7.31) (which is valid also for the case $0<\alpha \leq 1$ ), we have

$$
\begin{aligned}
\left\|\partial_{Y}\left(\Phi-\varphi_{1}-\psi_{0}\right)\right\| & \leq C \varepsilon^{\frac{1}{3}}\left\|\partial_{Y} \varphi_{1}\right\|+\frac{C}{\varepsilon^{\frac{1}{3}}}\left(\left\|\psi_{0}\right\|+\frac{1}{\varepsilon^{\frac{1}{3}}}\left\|U_{s} \psi_{0}\right\|\right), \\
\alpha\left\|\Phi-\varphi_{1}-\psi_{0}\right\| & \leq C \alpha \varepsilon^{\frac{1}{3}}\left\|U_{s}^{\prime \prime} \varphi_{1}\right\|+C \alpha\left\|\psi_{0}\right\|, \\
\left\|\left(\partial_{Y}^{2}-\alpha^{2}\right)\left(\Phi-\varphi_{1}-\psi_{0}\right)\right\| & \leq C\left\|\partial_{Y} \varphi_{1}\right\|+\frac{C}{\varepsilon^{\frac{2}{3}}}\left\|\psi_{0}\right\| .
\end{aligned}
$$

The proof is complete.

Proposition 6.1 gives two kinds of the estimates for $\psi_{0}$ :

$$
\begin{gathered}
\left\|U_{s} \psi_{0}\right\|+\varepsilon^{\frac{1}{3}}\left\|\psi_{0}\right\|+\varepsilon^{\frac{2}{3}}\left\|\partial_{Y} \psi_{0}\right\| \leq C \varepsilon\left\|\frac{f}{U_{s}}\right\|, \\
\varepsilon^{\frac{1}{3}}\left\|U_{s} \psi_{0}\right\|+\varepsilon^{\frac{2}{3}}\left\|\psi_{0}\right\|+\varepsilon\left\|\partial_{Y} \psi_{0}\right\| \leq C \varepsilon\left\|\frac{Y}{U_{s}} f\right\| .
\end{gathered}
$$

Then, by applying Proposition 5.1 and Proposition 2.2 (1) for $\varphi_{1}$, Proposition 7.3 finally yields the following corollaries in the case $\alpha \varepsilon^{\frac{1}{3}} \leq 1$. 
Corollary 7.5. Let $\varepsilon_{1}>0$ be the number in Proposition 7.3, and let $0<\varepsilon \leq \varepsilon_{1}$ and $0<\alpha \varepsilon^{\frac{1}{3}} \leq 1$. Let $f / U_{s} \in L^{2}\left(\mathbb{R}_{+}\right)$. Then the solution $\Phi$ in Proposition 7.8 satisfies the following estimates.

(i) when $\alpha \geq 1$,

$$
\begin{aligned}
\left\|\partial_{Y} \Phi\right\|+\alpha\|\Phi\| & \leq C \min \left\{\left\|\frac{Y}{U_{s}} f\right\|, \frac{1}{\alpha}\left\|\frac{f}{U_{s}}\right\|\right\}, \\
\left\|\left(\partial_{Y}^{2}-\alpha^{2}\right) \Phi\right\| & \leq C \min \left\{\left\|\frac{Y}{U_{s}} f\right\|, \frac{1}{\alpha}\left\|\frac{f}{U_{s}}\right\|\right\}+C\left\|\frac{f}{U_{s}}\right\| .
\end{aligned}
$$

(ii) when $0<\alpha \leq 1$, if $(1+Y)^{2} f \in L^{2}\left(\mathbb{R}_{+}\right)$in addition,

$$
\begin{aligned}
\alpha\|\Phi\| & \leq C \alpha\|(1+Y) \sigma[f]\|+C \alpha \varepsilon^{\frac{1}{3}}\|(1+Y) f\|+\frac{C}{\alpha^{\frac{1}{2}}}\left|\int_{0}^{\infty} f d Y\right|, \\
\left\|\partial_{Y} \Phi\right\| & \leq C\left\|(1+Y)^{2} f\right\|+\frac{C}{\alpha}\left|\int_{0}^{\infty} f d Y\right|, \\
\left\|\left(\partial_{Y}^{2}-\alpha^{2}\right) \Phi\right\| & \leq C\left\|(1+Y)^{2} f\right\|+\frac{C}{\alpha}\left|\int_{0}^{\infty} f d Y\right|+C\left\|\frac{f}{U_{s}}\right\| .
\end{aligned}
$$

Here $\sigma[f](Y)=\int_{Y}^{\infty} f d Y_{1}$.

In Corollary 7.5 the $H^{2}$ norm of the solution $\Phi$ is estimated uniformly in the small number $\varepsilon>0$, but under the condition of $f / U_{s} \in L^{2}\left(\mathbb{R}_{+}\right)$which implicitly imposes that $f$ vanishes on the boundary. By using the weak formulation (7.8) and the standard density argument, we have another $\epsilon$-dependent bound for the $H^{2}$ norm of $\Phi$ when $f$ does not necessarily vanish on the boundary. Precisely, the result is stated as follows.

Corollary 7.6. Let $\varepsilon_{1}>0$ be the number in Proposition 7.3, and let $0<\varepsilon \leq \varepsilon_{1}$ and $0<\alpha \varepsilon^{\frac{1}{3}} \leq 1$. Let $Y f / U_{s} \in L^{2}\left(\mathbb{R}_{+}\right)$. Then there exists a solution $\Phi \in H^{4}\left(\mathbb{R}_{+}\right) \cap H_{0}^{1}\left(\mathbb{R}_{+}\right)$to (7.7) satisfying the following estimates.

(i) when $\alpha \geq 1$,

$$
\begin{aligned}
\left\|\partial_{Y} \Phi\right\|+\alpha\|\Phi\| & \leq C\left\|\frac{Y}{U_{s}} f\right\|, \\
\left\|\left(\partial_{Y}^{2}-\alpha^{2}\right) \Phi\right\| & \leq \frac{C}{\varepsilon^{\frac{1}{3}}}\left(\left\|\frac{Y}{U_{s}} f\right\|+\|f\|\right) .
\end{aligned}
$$

(ii) when $0<\alpha \leq 1$, if $(1+Y)^{2} f \in L^{2}\left(\mathbb{R}_{+}\right)$in addition,

$$
\begin{aligned}
\alpha\|\Phi\| & \leq C \alpha\|(1+Y) \sigma[f]\|+C \alpha \varepsilon^{\frac{1}{3}}\|(1+Y) f\|+\frac{C}{\alpha^{\frac{1}{2}}}\left|\int_{0}^{\infty} f d Y\right|, \\
\left\|\partial_{Y} \Phi\right\| & \leq C\left\|(1+Y)^{2} f\right\|+\frac{C}{\alpha}\left|\int_{0}^{\infty} f d Y\right|, \\
\left\|\left(\partial_{Y}^{2}-\alpha^{2}\right) \Phi\right\| & \leq \frac{C}{\varepsilon^{\frac{1}{3}}}\left(\left\|(1+Y)^{2} f\right\|+\frac{C}{\alpha}\left|\int_{0}^{\infty} f d Y\right|\right) .
\end{aligned}
$$

Here $\sigma[f](Y)=\int_{Y}^{\infty} f d Y_{1}$.

Remark 7.7. Corollary 7.6 implies that $\varphi_{1}+\psi_{0} \in H^{2}\left(\mathbb{R}_{+}\right)$even when $f$ does not vanish on the boundary, though neither $\varphi_{1}$ or $\psi_{0}$ belongs to $H^{2}\left(\mathbb{R}_{+}\right)$for such a case. 
Proof. Thanks to Corollary 7.5, it suffices to show the estimates (7.45) and (7.48) for the solution $\Phi$ obtained in Corollary 7.5. Then Corollary 7.6 follows from the density argument. Let $\Phi \in H^{4}\left(\mathbb{R}_{+}\right) \cap H_{0}^{1}\left(\mathbb{R}_{+}\right),\left.\partial_{Y} H_{\alpha} \Phi\right|_{Y=0}=0$, be the solution to (7.8) obtained in Corollary 7.5. Then $\Psi=H_{\alpha} \Phi$ solves the Airy equation Airy $[\Psi]=U_{s}^{\prime \prime} \Phi+f \in L^{2}\left(\mathbb{R}_{+}\right)$subject to the Neumann boundary condition $\left.\partial_{Y} \Psi\right|_{Y=0}=0$. Thus we have from the integration by parts,

$$
\left\|\sqrt{U_{s}} \Psi\right\|^{2}-i \varepsilon\left(\left\|\partial_{Y} \Psi\right\|^{2}+\alpha^{2}\|\Psi\|^{2}\right)=\left\langle U_{s}^{\prime \prime} \Phi+f, \Psi\right\rangle .
$$

Hence the interpolation inequality

$$
\|\Psi\|^{2} \leq C\left\|\sqrt{U_{s}} \Psi\right\|^{\frac{4}{3}}\left\|\partial_{Y} \Psi\right\|^{\frac{2}{3}}+C\left\|\sqrt{U_{s}} \Psi\right\|^{2}
$$

implies

$$
\|\Psi\| \leq \frac{C}{\varepsilon^{\frac{1}{3}}}\left\|U_{s}^{\prime \prime} \Phi+f\right\|
$$

as in the proof of Proposition 6.1. Since $\left\|U_{s}^{\prime \prime} \Phi\right\| \leq C\left\|\partial_{Y} \Phi\right\|$ by the Hardy inequality, estimates (7.45) and (7.48) follow from the estimate of $\left\|\partial_{Y} \Phi\right\|$ in Corollary 7.5. The proof is complete.

\subsection{Construction of a boundary corrector}

Let $\Phi_{\text {slip }}[f]$ be the solution to the modified Orr-Sommerfeld equation (7.7) obtained by Proposition 7.3. Then the solution to the original problem (7.1) is obtained by solving the equation

$$
\left\{\begin{array}{l}
O S[\phi]=0, \quad Y>0, \\
\left.\phi\right|_{Y=0}=0,\left.\quad \partial_{Y} \phi\right|_{Y=0}=-\left.\partial_{Y} \Phi_{s l i p}[f]\right|_{Y=0} .
\end{array}\right.
$$

Remark 7.8. By interpolation of (7.44) and (7.45), or (7.47) and (7.48), one has

$$
\begin{array}{rr}
\left|\partial_{Y} \Phi_{\text {slip }}[f]\right|_{Y=0} \mid \leq \frac{C}{\varepsilon^{\frac{1}{6}}}\left(\left\|\frac{Y}{U_{s}} f\right\|+\|f\|\right) & \text { if } \alpha \geq 1, \\
\left|\partial_{Y} \Phi_{\text {slip }}[f]\right|_{Y=0} \mid \leq \frac{C}{\varepsilon^{\frac{1}{6}}}\left(\left\|(1+Y)^{2} f\right\|+\frac{1}{\alpha}\left|\int_{0}^{\infty} f d Y\right|\right) & \text { if } 0<\alpha \leq 1 .
\end{array}
$$

\subsection{Slow mode}

In this subsection we construct a solution $\phi_{\text {slow }}$ to the equation

$$
\left\{\begin{array}{l}
O S[\phi]=0, \quad Y>0 \\
\left.\phi\right|_{Y=0}=1+\text { small order }
\end{array}\right.
$$

decaying as $Y \rightarrow \infty$ around the Rayleigh solution $\varphi_{\text {slow, Ray }}$ obtained in Corollary 5.5 and Proposition 5.6. This solution is called the slow mode, and will take the form $\phi_{\text {slow }}=$ $\varphi_{\text {slow, Ray }}+\tilde{\phi}_{\text {slow }}$.

For the moment we assume that $\frac{U_{s}^{\prime \prime}}{U_{s}} \in L^{2}\left(\mathbb{R}_{+}\right)$, which ensures the $H^{2}$ regularity of $\varphi_{\text {slow, Ray }}$ and justifies the formal computation in various steps. Later we shall recover the $H^{2}$ regularity of $\phi_{\text {slow }}$ which does not depend on the condition $\frac{U_{s}^{\prime \prime}}{U_{s}} \in L^{2}\left(\mathbb{R}_{+}\right)$. Then the standard limiting 
process gives the result for the general case without the condition $\frac{U_{s}^{\prime \prime}}{U_{s}} \in L^{2}\left(\mathbb{R}_{+}\right)$. Firstly we observe that $\varphi_{\text {slow, Ray }}$ satisfies the identity

$$
\begin{aligned}
& \left\langle\text { Ray }\left[\varphi_{\text {slow }, \text { Ray }}\right], q\right\rangle+i \varepsilon\left\langle\left(\partial_{Y}^{2}-\alpha^{2}\right) \varphi_{\text {slow,Ray }},\left(\partial_{Y}^{2}-\alpha^{2}\right) q\right\rangle \\
& =i \varepsilon\left\langle\left(\partial_{Y}^{2}-\alpha^{2}\right) \varphi_{\text {slow }, \text { Ray }},\left(\partial_{Y}^{2}-\alpha^{2}\right) q\right\rangle \\
& =i \varepsilon\left\langle\frac{1}{U_{s}} \operatorname{Ray}\left[\varphi_{\text {slow }, \text { Ray }}\right]+\frac{U_{s}^{\prime \prime}}{U_{s}} \varphi_{\text {slow }, \text { Ray }},\left(\partial_{Y}^{2}-\alpha^{2}\right) q\right\rangle \\
& =i \varepsilon\left\langle\frac{U_{s}^{\prime \prime}}{U_{s}} \varphi_{\text {slow }, \text { Ray }},\left(\partial_{Y}^{2}-\alpha^{2}\right) q\right\rangle, \quad q \in H^{2}\left(\mathbb{R}_{+}\right) .
\end{aligned}
$$

In this computation we are using the condition $\frac{U_{s}^{\prime \prime}}{U_{s}} \in L^{2}\left(\mathbb{R}_{+}\right)$so that each term makes sense for any $q \in H^{2}\left(\mathbb{R}_{+}\right)$. By the ansatz $\phi_{\text {slow }}=\varphi_{\text {slow, Ray }}+\tilde{\phi}_{\text {slow }}$ we shall construct $\tilde{\phi}_{\text {slow }} \in H^{2}\left(\mathbb{R}_{+}\right) \cap H_{0}^{1}\left(\mathbb{R}_{+}\right)$as the solution to

$$
\begin{gathered}
\left\langle\operatorname{Ray}\left[\tilde{\phi}_{\text {slow }}\right], q\right\rangle+i \varepsilon\left\langle\left(\partial_{Y}^{2}-\alpha^{2}\right) \tilde{\phi}_{\text {slow }},\left(\partial_{Y}^{2}-\alpha^{2}\right) q\right\rangle=-i \varepsilon\left\langle\frac{U_{s}^{\prime \prime}}{U_{s}} \varphi_{\text {slow }, R a y},\left(\partial_{Y}^{2}-\alpha^{2}\right) q\right\rangle, \\
q \in H^{2}\left(\mathbb{R}_{+}\right), \quad \partial_{Y} q(0)=0 .
\end{gathered}
$$

To this end, by using $O S=\left(\partial_{Y}^{2}-\alpha^{2}\right)$ Airy $-2 \partial_{Y}\left(U_{s}^{\prime} \cdot\right)$ we take $\psi_{\text {slow }} \in H^{2}\left(\mathbb{R}_{+}\right) \cap H_{0}^{1}\left(\mathbb{R}_{+}\right)$as the solution to

$$
\operatorname{Airy}\left[\psi_{\text {slow }}\right]=i \varepsilon \frac{U_{s}^{\prime \prime}}{U_{s}} \varphi_{\text {slow }, \text { Ray }}
$$

Then we see

$$
\begin{aligned}
& \left\langle\operatorname{Ray}\left[\psi_{\text {slow }}\right], q\right\rangle+i \varepsilon\left\langle\left(\partial_{Y}^{2}-\alpha^{2}\right) \psi_{\text {slow }},\left(\partial_{Y}^{2}-\alpha^{2}\right) q\right\rangle \\
& =\left\langle\text { Airy }\left[\psi_{\text {slow }}\right],\left(\partial_{Y}^{2}-\alpha^{2}\right) q\right\rangle-2\left\langle\partial_{Y}\left(U_{s}^{\prime} \psi_{\text {slow }}\right), q\right\rangle \\
& =i \varepsilon\left\langle\frac{U_{s}^{\prime \prime}}{U_{s}} \varphi_{\text {slow }, \text { Ray }},\left(\partial_{Y}^{2}-\alpha^{2}\right) q\right\rangle-2\left\langle\partial_{Y}\left(U_{s}^{\prime} \psi_{\text {slow }}\right), q\right\rangle, \quad q \in H^{2}\left(\mathbb{R}_{+}\right) .
\end{aligned}
$$

Finally we take $\Phi_{\text {slow }} \in H^{4}\left(\mathbb{R}_{+}\right) \cap H_{0}^{1}\left(\mathbb{R}_{+}\right)$as the solution to (7.7) with the source term $2 \partial_{Y}\left(U_{s}^{\prime} \psi_{\text {slow }}\right)$, which is constructed in Corollary 7.6. Then $\tilde{\phi}_{\text {slow }}$ is constructed in the form $\tilde{\phi}_{\text {slow }}=\psi_{\text {slow }}+\Phi_{\text {slow }} \in H^{2}\left(\mathbb{R}_{+}\right) \cap H_{0}^{1}\left(\mathbb{R}_{+}\right)$. Moreover, the function

$$
\phi_{\text {slow }}=\varphi_{\text {slow }, \text { Ray }}+\psi_{\text {slow }}+\Phi_{\text {slow }} \in H^{2}\left(\mathbb{R}_{+}\right)
$$

satisfies $O S\left[\phi_{\text {slow }}\right]=0$ in the weak form:

$$
\begin{gathered}
\left\langle U_{s}\left(\partial_{Y}^{2}-\alpha^{2}\right) \phi_{\text {slow }}-U_{s}^{\prime \prime} \phi_{\text {slow }}, q\right\rangle+i \varepsilon\left\langle\left(\partial_{Y}^{2}-\alpha^{2}\right) \phi_{\text {slow }},\left(\partial_{Y}^{2}-\alpha^{2}\right) q\right\rangle=0, \\
q \in H^{2}\left(\mathbb{R}_{+}\right), \quad \partial_{Y} q(0)=0 .
\end{gathered}
$$

The requirement $\partial_{Y} q(0)=0$ for the test function $q$ is due to the weak formulation of $\Phi_{\text {slow }}$ as in (7.8). The identity (7.55) implies that $\Psi_{\text {slow }}=\left(\partial_{Y}^{2}-\alpha^{2}\right) \phi_{\text {slow }}$ is the very weak solution to the Poisson equation $i \varepsilon\left(\partial_{Y}^{2}-\alpha^{2}\right) \Psi_{\text {slow }}=-U_{s} \Psi_{\text {slow }}+U_{s}^{\prime \prime} \phi_{\text {slow }}$ subject to the Neumann boundary condition $\left.\partial_{Y} \Psi_{\text {slow }}\right|_{Y=0}=0$. Hence $\Psi_{\text {slow }}$ belongs to $H^{2}\left(\mathbb{R}_{+}\right)$by the elliptic regularity and satisfies the Airy equation $\operatorname{Airy}\left[\Psi_{\text {slow }}\right]=U_{s}^{\prime \prime} \phi_{\text {slow }}$ with the zero Neumann boundary condition. Then we have

$$
\left\langle U_{s} \Psi_{\text {slow }}-U_{s}^{\prime \prime} \phi_{\text {slow }}, \Psi_{\text {slow }}\right\rangle-i \varepsilon\left(\left\|\partial_{Y} \Psi_{\text {slow }}\right\|^{2}+\alpha^{2}\left\|\Psi_{\text {slow }}\right\|^{2}\right)=0,
$$


which yields, as in the proof of Corollary 7.6,

$$
\left\|\left(\partial_{Y}^{2}-\alpha^{2}\right) \phi_{\text {slow }}\right\|=\left\|\Psi_{\text {slow }}\right\| \leq \frac{C}{\varepsilon^{\frac{1}{3}}}\left\|U_{s}^{\prime \prime} \phi_{\text {slow }}\right\| .
$$

Hence, the $H^{2}$ regularity of $\phi_{\text {slow }}$ is estimated in terms of $\left\|U_{s}^{\prime \prime} \phi_{\text {slow }}\right\|$, for which the condition $\frac{U_{s}^{\prime \prime}}{U_{s}} \in L^{2}\left(\mathbb{R}_{+}\right)$is not required. Now it suffices to establish the estimates of $\phi_{\text {slow }}=\varphi_{\text {slow, Ray }}+$ $\tilde{\phi}_{\text {slow }}=\varphi_{\text {slow, Ray }}+\psi_{\text {slow }}+\Phi_{\text {slow }}$, which will be considered below depending on the two cases $0<\alpha \leq 1$ and $\alpha \geq 1$.

\subsubsection{Estimates in the case $0<\alpha \leq 1$}

Let us estimate $\tilde{\phi}_{\text {slow }}$, which is equal to $\psi_{\text {slow }}+\Phi_{\text {slow }} \in H^{2}\left(\mathbb{R}_{+}\right) \cap H_{0}^{1}\left(\mathbb{R}_{+}\right)$. Since the function $\psi_{\text {slow }}$ satisfies Airy $\left[\psi_{\text {slow }}\right]=i \varepsilon \frac{U_{s}^{\prime \prime}}{U_{s}} \varphi_{\text {slow, Ray }}$ we have from $\varphi_{\text {slow, Ray }}=\frac{c_{E}}{\alpha} U_{s} e^{-\alpha Y}+\varphi_{s R a y, 1}+$ $\varphi_{\text {sRay }, 2}$,

$$
\psi_{\text {slow }}=\psi_{\text {slow }, 0}+\psi_{\text {slow }, 1},
$$

where $\psi_{\text {slow }, 0} \in H^{2}\left(\mathbb{R}_{+}\right) \cap H_{0}^{1}\left(\mathbb{R}_{+}\right)$is the solution to

$$
\operatorname{Airy}\left[\psi_{\text {slow }, 0}\right]=i \varepsilon \frac{c_{E}}{\alpha} U_{s}^{\prime \prime} e^{-\alpha Y},\left.\quad \psi_{\text {slow }, 0}\right|_{Y=0}=0,
$$

while $\psi_{\text {slow }, 1} \in H^{2}\left(\mathbb{R}_{+}\right) \cap H_{0}^{1}\left(\mathbb{R}_{+}\right)$is the Airy solution with the source $i \varepsilon \frac{U_{s}^{\prime \prime}}{U_{s}}\left(\varphi_{s R a y, 1}+\varphi_{s R a y, 2}\right)$. As for the estimates of $\psi_{\text {slow }, 0}$ we have from Proposition 6.1,

$$
\left\|\partial_{Y} \psi_{\text {slow }, 0}\right\| \leq C \varepsilon^{\frac{1}{3}}\left\|\frac{c_{E}}{\alpha} U_{s}^{\prime \prime} e^{-\alpha Y}\right\| \leq \frac{C \varepsilon^{\frac{1}{3}}}{\alpha},
$$

and

$$
\alpha\left\|\psi_{\text {slow }, 0}\right\| \leq C \alpha \varepsilon^{\frac{2}{3}}\left\|\frac{c_{E}}{\alpha} U_{s}^{\prime \prime} e^{-\alpha Y}\right\| \leq C \varepsilon^{\frac{2}{3}} .
$$

As for the estimates of $\psi_{\text {slow, } 1}$, in virtue of (6.4) of Proposition 6.1, we see

$$
\left\|\partial_{Y} \psi_{\text {slow }, 1}\right\| \leq C\left\|\frac{Y U_{s}^{\prime \prime}}{U_{s}}\left(\varphi_{s R a y, 1}+\varphi_{\text {sRay }, 2}\right)\right\| \leq C .
$$

and

$$
\alpha\left\|\psi_{\text {slow }, 1}\right\| \leq C \alpha \varepsilon^{\frac{1}{3}}\left\|\frac{Y U_{s}^{\prime \prime}}{U_{s}}\left(\varphi_{\text {sRay }, 1}+\varphi_{\text {sRay }, 2}\right)\right\| \leq C \alpha \varepsilon^{\frac{1}{3}},
$$

In order to estimate $\Phi_{\text {slow }}$, the important point is that $\int_{0}^{\infty} \partial_{Y}\left(U_{s}^{\prime} \psi_{\text {slow }}\right) d Y=0$ in virtue of $\psi_{\text {slow }} \in H_{0}^{1}\left(\mathbb{R}_{+}\right)$, and thus, Corollary 7.6 yields the estimates of $\Phi_{\text {slow }}$. Let us decompose $\Phi_{\text {slow }}$ as $\Phi_{\text {slow }}=\Phi_{\text {slow }, 0}+\Phi_{\text {slow }, 1}$ according to the decomposition of the source term $2 \partial_{Y}\left(U_{s}^{\prime} \psi_{\text {slow }, 0}+\right.$ $\left.U_{s}^{\prime} \psi_{\text {slow }, 1}\right)$. Then we have from Corollary 7.6,

$$
\left\|\partial_{Y} \Phi_{\text {slow }, 0}\right\|+\alpha\left\|\Phi_{\text {slow }, 0}\right\| \leq C\left\|(1+Y)^{2} \partial_{Y}\left(U_{s}^{\prime} \psi_{\text {slow }, 0}\right)\right\| \leq C\left\|\partial_{Y} \psi_{\text {slow }, 0}\right\| \leq \frac{C \varepsilon^{\frac{1}{3}}}{\alpha} .
$$

Similarly, we have for $\psi_{\text {slow }, 1}$,

$$
\begin{aligned}
\left\|\partial_{Y} \Phi_{\text {slow }, 1}\right\|+\alpha\left\|\Phi_{\text {slow }, 1}\right\| & \leq C\left\|(1+Y)^{2} \partial_{Y}\left(U_{s}^{\prime} \psi_{\text {slow }, 1}\right)\right\| \\
& \leq C\left\|\partial_{Y} \psi_{\text {slow }, 1}\right\| \leq C
\end{aligned}
$$


Collecting these above, we have for $\tilde{\phi}_{\text {slow }}=\psi_{\text {slow }}+\Phi_{\text {slow }}=\psi_{\text {slow }, 0}+\psi_{\text {slow }, 1}+\Phi_{\text {slow }, 0}+\Psi_{\text {slow }, 1}$,

$$
\left\|\partial_{Y} \tilde{\phi}_{\text {slow }}\right\|+\alpha\left\|\tilde{\phi}_{\text {slow }}\right\| \leq C\left(\frac{\varepsilon^{\frac{1}{3}}}{\alpha}+1\right),
$$

The next step is to estimate $\left\|\left(\partial_{Y}^{2}-\alpha^{2}\right) \phi_{\text {slow,re }}\right\|$, where

$$
\phi_{\text {slow }, \text { re }}=\phi_{\text {slow }}-\frac{c_{E}}{\alpha} U_{s} e^{-\alpha Y} .
$$

Combined with the estimate on $\left\|\partial_{Y} \phi_{\text {slow,re }}\right\|$, that can be deduced from the previous bounds, we will obtain by interpolation an $L^{\infty}$ bound on $\partial_{Y} \phi_{\text {slow,re }}$. The point is to show that $\partial_{Y} \phi_{\text {slow, re }}(0) \ll \partial_{Y}\left(\frac{c_{E}}{\alpha} U_{s} e^{-\alpha Y}\right)(0)$ when $\alpha \ll 1$. Unfortunately, (7.57) is not accurate enough for this purpose, and we shall rather make use of (7.56).

Set $\Psi_{\text {slow,re }}=\left(\partial_{Y}^{2}-\alpha^{2}\right) \phi_{\text {slow,re }}$. We observe that

$$
\begin{aligned}
\Psi_{\text {slow }} & =\left(\partial_{Y}^{2}-\alpha^{2}\right) \phi_{\text {slow }}=\frac{c_{E}}{\alpha}\left(U_{s}^{\prime \prime} e^{-\alpha Y}-2 \alpha U_{s}^{\prime} e^{-\alpha Y}\right)+\Psi_{\text {slow }, \text { re }}, \\
U_{s} \Psi_{\text {slow }}-U_{s}^{\prime \prime} \phi_{\text {slow }} & =-2 c_{E} U_{s} U_{s}^{\prime} e^{-\alpha Y}+U_{s} \Psi_{\text {slow }, r e}-U_{s}^{\prime \prime} \phi_{\text {slow }, r e}
\end{aligned}
$$

Thus (7.56) gives

$$
\begin{aligned}
& \left\langle-2 c_{E} U_{s} U_{s}^{\prime} e^{-\alpha Y}+U_{s} \Psi_{\text {slow }, r e}-U_{s}^{\prime \prime} \phi_{\text {slow }, r e}, \frac{c_{E}}{\alpha}\left(U_{s}^{\prime \prime} e^{-\alpha Y}-2 \alpha U_{s}^{\prime} e^{-\alpha Y}\right)+\Psi_{\text {slow }, r e}\right\rangle \\
& -i \varepsilon\left(\left\|\partial_{Y}\left(\frac{c_{E}}{\alpha} U_{s} e^{-\alpha Y}\right)\right\|^{2}+\alpha^{2}\left\|\frac{c_{E}}{\alpha} U_{s} e^{-\alpha Y}\right\|^{2}+\left\|\partial_{Y} \Psi_{\text {slow }, r e}\right\|^{2}+\alpha^{2}\left\|\Psi_{\text {slow }, \text { re }}\right\|^{2}\right. \\
& \left.\quad+2 \operatorname{Re}\left\langle\partial_{Y}\left(\frac{c_{E}}{\alpha} U_{s} e^{-\alpha Y}\right), \partial_{Y} \Psi_{\text {slow }, r e}\right\rangle+2 \alpha^{2} \operatorname{Re}\left\langle\frac{c_{E}}{\alpha} e^{-\alpha Y}, \Psi_{\text {slow }, r e}\right\rangle\right) \\
& =0 .
\end{aligned}
$$

The real part of this identity gives

$$
\begin{aligned}
\left\|\sqrt{U_{s}} \Psi_{\text {slow }, \text { re }}\right\|^{2}= & \operatorname{Re}\left\langle 2 c_{E} U_{s} U_{s}^{\prime} e^{-\alpha Y}+U_{s}^{\prime \prime} \phi_{\text {slow }, \text { re }}, \Psi_{\text {slow }, r e}\right\rangle \\
- & \operatorname{Re}\left\langle U_{s} \Psi_{\text {slow }, r e}, \frac{c_{E}}{\alpha}\left(U_{s}^{\prime \prime} e^{-\alpha Y}-2 \alpha U_{s}^{\prime} e^{-\alpha Y}\right)\right\rangle \\
& +\operatorname{Re}\left\langle 2 c_{E} U_{s} U_{s}^{\prime} e^{-\alpha Y}+U_{s}^{\prime \prime} \phi_{\text {slow }, r e}, \frac{c_{E}}{\alpha}\left(U_{s}^{\prime \prime} e^{-\alpha Y}-2 \alpha U_{s}^{\prime} e^{-\alpha Y}\right)\right\rangle,
\end{aligned}
$$

which implies

$$
\begin{aligned}
\left\|\sqrt{U_{s}} \Psi_{\text {slow }, r e}\right\|^{2} \leq & C\left\|\sqrt{U_{s}} U_{s}^{\prime} e^{-\alpha Y}\right\|^{2}+\left\|U_{s}^{\prime \prime} \phi_{\text {slow }, r e}\right\|\left\|\Psi_{\text {slow }, \text { re }}\right\| \\
+ & \frac{C}{\alpha^{2}}\left\|\sqrt{U_{s}}\left(U_{s}^{\prime \prime} e^{-\alpha Y}-2 \alpha U_{s}^{\prime} e^{-\alpha Y}\right)\right\|^{2} \\
& +\frac{C}{\alpha}\left(1+\left\|U_{s}^{\prime \prime} \phi_{\text {slow }, r e}\right\|\right) \\
\leq & \frac{C}{\alpha^{2}}+\left\|U_{s}^{\prime \prime} \phi_{\text {slow }, \text { re }}\right\|\left(\left\|\Psi_{\text {slow }, \text { re }}\right\|+\left\|U_{s}^{\prime \prime} \phi_{\text {slow }, r e}\right\|\right) .
\end{aligned}
$$

Recall that $\phi_{\text {slow }, r e}=\varphi_{\text {sRay }, 1}+\varphi_{\text {sRay }, 2}+\tilde{\phi}_{\text {slow }}$, and thus, by Corollary 5.5 and (7.60),

$$
\left\|U_{s}^{\prime \prime} \phi_{s l o w, r e}\right\| \leq C\left(\frac{\varepsilon^{\frac{1}{3}}}{\alpha}+1\right) .
$$

Then we have

$$
\left\|\sqrt{U_{s}} \Psi_{\text {slow }, r e}\right\|^{2} \leq \frac{C}{\alpha^{2}}+C\left(\frac{\varepsilon^{\frac{1}{3}}}{\alpha}+1\right)\left\|\Psi_{\text {slow }, r e}\right\|
$$


On the other hand, the imaginary part of (7.61) yields, since $\left\langle-2 c_{E} U_{s} U_{s}^{\prime} e^{-\alpha Y}, \frac{c_{E}}{\alpha}\left(U_{s}^{\prime \prime} e^{-\alpha Y}-\right.\right.$ $\left.\left.2 \alpha U_{s}^{\prime} e^{-\alpha Y}\right)\right\rangle \in \mathbb{R}$,

$$
\begin{aligned}
& \varepsilon\left(\left\|\partial_{Y} \Psi_{\text {slow }, r e}\right\|^{2}+\alpha^{2}\left\|\Psi_{\text {slow }, r e}\right\|^{2}\right) \\
\leq & \frac{C \varepsilon}{\alpha^{2}}+\frac{C}{\alpha}\left\|\sqrt{U_{s}} \Psi_{\text {slow }, r e}\right\|+\frac{C}{\alpha}\left\|U_{s}^{\prime \prime} \phi_{\text {slow }, r e}\right\|+C\left\|\Psi_{\text {slow }, r e}\right\|+C\left\|U_{s}^{\prime \prime} \phi_{\text {slow }, \text { re }}\right\|\left\|\Psi_{\text {slow }, \text { re }}\right\| \\
\leq & \frac{C}{\alpha^{2}}+C\left(\frac{\varepsilon^{\frac{1}{3}}}{\alpha}+1\right)\left\|\Psi_{\text {slow }, r e}\right\| .
\end{aligned}
$$

Thus the inequality

$$
\left\|\Psi_{\text {slow }, \text { re }}\right\|^{2} \leq C\left\|\sqrt{U_{s}} \Psi_{\text {slow }, r e}\right\|^{\frac{4}{3}}\left\|\partial_{Y} \Psi_{\text {slow, re }}\right\|^{\frac{2}{3}}+C\left\|\sqrt{U_{s}} \Psi_{\text {slow }, \text { re }}\right\|^{2},
$$

combined with (7.63) and (7.64), leads to

$$
\left\|\Psi_{\text {slow }, \text { re }}\right\|^{2} \leq \frac{C}{\varepsilon^{\frac{1}{3}}}\left(\frac{1}{\alpha^{2}}+\left(\frac{\varepsilon^{\frac{1}{3}}}{\alpha}+1\right)\left\|\Psi_{\text {slow }, \text { re }}\right\|\right) \leq \frac{C}{\varepsilon^{\frac{1}{3}} \alpha^{2}}+\frac{C}{\varepsilon^{\frac{2}{3}}}\left(\frac{\varepsilon^{\frac{1}{3}}}{\alpha}+1\right)^{2} .
$$

Hence we have arrived at

$$
\left\|\left(\partial_{Y}^{2}-\alpha^{2}\right) \phi_{\text {slow }, r e}\right\|=\left\|\Psi_{\text {slow }, r e}\right\| \leq \frac{C}{\varepsilon^{\frac{1}{6}} \alpha}+\frac{C}{\varepsilon^{\frac{1}{3}}} .
$$

The above estimates are valid without the condition $\frac{U_{s}^{\prime \prime}}{U_{s}} \in L^{2}\left(\mathbb{R}_{+}\right)$. We summarize the above results as follows.

Proposition 7.9. Let $0<\varepsilon \leq \varepsilon_{1}$ and $0<\alpha \leq 1$. Then there exists a solution $\phi_{\text {slow }} \in$ $H^{4}\left(\mathbb{R}_{+}\right)$to $O S\left[\phi_{\text {slow }}\right]=0$ satisfying the following properties: $\phi_{\text {slow }}=\frac{c_{F}}{\alpha} U_{s} e^{-\alpha Y}+\phi_{\text {slow }, \text { re }}$, where

$$
\phi_{\text {slow }}(0)=1
$$

and

$$
\begin{aligned}
\left\|\partial_{Y} \phi_{\text {slow }, r e}\right\|+\alpha\left\|\phi_{\text {slow }, r e}\right\| & \leq C\left(\frac{\varepsilon^{\frac{1}{3}}}{\alpha}+1\right), \\
\left\|\partial_{Y} \phi_{\text {slow }, r e}\right\|_{L^{\infty}} & \leq C\left(\frac{\varepsilon^{\frac{1}{12}}}{\alpha}+\frac{1}{\varepsilon^{\frac{1}{4}}}\right), \\
\left\|\left(\partial_{Y}^{2}-\alpha^{2}\right) \phi_{\text {slow }, r e}\right\| & \leq C\left(\frac{1}{\varepsilon^{\frac{1}{6}} \alpha}+\frac{1}{\varepsilon^{\frac{1}{3}}}\right) .
\end{aligned}
$$

In particular, we have

$$
\partial_{Y} \phi_{\text {slow }}(0)=\frac{c_{E} U_{s}^{\prime}(0)}{\alpha}+O\left(\frac{\varepsilon^{\frac{1}{12}}}{\alpha}+\frac{1}{\varepsilon^{\frac{1}{4}}}\right) .
$$

Proof. It suffices to recall $\phi_{\text {slow }, r e}=\varphi_{\text {sRay }, 1}+\varphi_{\text {sRay }, 2}+\tilde{\phi}_{\text {slow }}$. Note that $\varphi_{\text {sRay }, 2}, \tilde{\phi}_{\text {slow }}$ belong to $H_{0}^{1}\left(\mathbb{R}_{+}\right)$and that $U_{s}(0)=0$, and thus, $\phi_{\text {slow }}(0)=\phi_{\text {slow,re }}(0)=\varphi_{\text {sRay }, 1}(0)=1$. This proves (7.67). Estimate (7.68) follows from Corollary 5.5 and (7.60), while (7.70) is proved in (7.66). Then (7.69) follows from the interpolation. The proof is complete. 


\subsubsection{Estimates in the case $\alpha \geq 1$}

Let us estimate $\tilde{\phi}_{\text {slow }}=\tilde{\psi}_{\text {slow }}+\tilde{\Phi}_{\text {slow }}$. Since $\tilde{\psi}_{\text {slow }}$ is the Airy solution with the source $f=i \varepsilon \frac{U_{s}^{\prime \prime}}{U_{s}} \varphi_{\text {slow, Ray }}$, we apply (6.4) of Proposition 6.1 to obtain

$$
\left\|\partial_{Y} \tilde{\psi}_{\text {slow }}\right\|+\alpha\left\|\tilde{\psi}_{\text {slow }}\right\| \leq C\left\|\frac{Y U_{s}^{\prime \prime}}{U_{s}} \varphi_{\text {slow,Ray }}\right\| \leq C .
$$

Next we recall that $\tilde{\Phi}_{\text {slow }}$ is the solution to $(7.7)$ with the source $f=2 \partial_{Y}\left(U_{s}^{\prime} \tilde{\psi}_{\text {slow }}\right)$, we have from Corollary 7.6,

$$
\left\|\partial_{Y} \tilde{\Phi}_{\text {slow }}\right\|+\alpha\left\|\tilde{\Phi}_{\text {slow }}\right\| \leq C\left\|(1+Y)^{2} \partial_{Y}\left(U_{s}^{\prime} \tilde{\psi}_{\text {slow }}\right)\right\| \leq C\left\|\partial_{Y} \tilde{\psi}_{\text {slow }}\right\| \leq C .
$$

Collecting these, we obtain

$$
\left\|\partial_{Y} \tilde{\phi}_{\text {slow }}\right\|+\alpha\left\|\tilde{\phi}_{\text {slow }}\right\| \leq C
$$

In the case $\alpha \geq 1$ we set $\phi_{\text {slow, re }}$ as

$$
\phi_{\text {slow }, \text { re }}=\phi_{\text {slow }}-e^{-\alpha Y} .
$$

Combining (7.57) and (7.72) with Proposition 5.6, we have

$$
\left\|\left(\partial_{Y}^{2}-\alpha^{2}\right) \phi_{\text {slow }, \text { re }}\right\|=\left\|\left(\partial_{Y}^{2}-\alpha^{2}\right) \phi_{\text {slow }}\right\| \leq \frac{C}{\varepsilon^{\frac{1}{3}}}\left(\left\|U_{s}^{\prime \prime} \varphi_{\text {slow }, \text { Ray }}\right\|+\left\|U_{s}^{\prime \prime} \tilde{\phi}_{\text {slow }}\right\|\right) \leq \frac{C}{\varepsilon^{\frac{1}{3}}} .
$$

Here $C$ is independent of the condition $\frac{U_{s}^{\prime \prime}}{U_{s}} \in L^{2}\left(\mathbb{R}_{+}\right)$. We summarize the above results as follows.

Proposition 7.10. Let $0<\varepsilon \leq \varepsilon_{1}, \alpha \geq 1$, and $\alpha \varepsilon^{\frac{1}{3}} \leq 1$. Then there exists a solution $\phi_{\text {slow }} \in H^{4}\left(\mathbb{R}_{+}\right)$to $O S\left[\phi_{\text {slow }}\right]=0$ satisfying the following properties: $\phi_{\text {slow }}=e^{-\alpha Y}+\phi_{\text {slow }, \text { re }}$ where $\phi_{\text {slow }, \text { re }} \in H^{4}\left(\mathbb{R}_{+}\right) \cap H_{0}^{1}\left(\mathbb{R}_{+}\right)$and

$$
\begin{aligned}
\left\|\partial_{Y} \phi_{\text {slow }, r e}\right\|+\alpha\left\|\phi_{\text {slow }, r e}\right\| & \leq C, \\
\left\|\partial_{Y} \phi_{\text {slow }, r e}\right\|_{L^{\infty}} & \leq \frac{C}{\varepsilon^{\frac{1}{6}}}, \\
\left\|\left(\partial_{Y}^{2}-\alpha^{2}\right) \phi_{\text {slow }, r e}\right\| & \leq \frac{C}{\varepsilon^{\frac{1}{3}}} .
\end{aligned}
$$

In particular, $\phi_{\text {slow }}(0)=1$ and $\left|\partial_{Y} \phi_{\text {slow }}(0)\right| \leq \alpha+C \varepsilon^{-\frac{1}{6}}$.

\subsection{Fast mode}

In this subsection we construct a solution $\phi_{\text {fast }}$ to

$$
\left\{\begin{array}{l}
O S[\phi]=0, \quad Y>0, \\
\left.\phi\right|_{Y=0}=O(1)
\end{array}\right.
$$

possessing the boundary layer structure, called the fast mode. To this end we first aim to construct the approximate solution to the problem

$$
\left\{\begin{array}{l}
\operatorname{Airy}[\psi]=0, \quad Y>0, \\
\left.\psi\right|_{Y=0}=O(1)
\end{array}\right.
$$


possessing the boundary layer structure. To this end we set

$$
\lambda=\frac{i U_{s}^{\prime}(0)}{\varepsilon} .
$$

Let $\Gamma(z)$ be the Gamma function and let $\operatorname{Ai}(z)$ be the Airy function which solves the equation $\frac{d^{2} \mathrm{Ai}}{d z^{2}}-z \mathrm{Ai}=0$ for $z \in \mathbb{C}$ and satisfies $\operatorname{Ai}(0)=\frac{1}{3^{\frac{2}{3}} \Gamma\left(\frac{2}{3}\right)}, \operatorname{Ai}^{\prime}(0)=-\frac{1}{3^{\frac{1}{3}} \Gamma\left(\frac{1}{3}\right)}$, and

$$
\operatorname{Ai}(z) \sim z^{-\frac{1}{4}} e^{-\frac{2}{3} z^{\frac{3}{2}}}, \quad|z| \gg 1, \quad|\arg z| \leq \pi-\theta, \theta \in(0, \pi) .
$$

See [1, Chapter 10] for details about the Airy function. Then we set

$$
\psi_{\text {fast }, 0}(Y)=\operatorname{Ai}\left(\lambda^{\frac{1}{3}} Y\right), \quad Y>0 .
$$

Here $\lambda^{\frac{1}{3}}=\left(\frac{U_{s}^{\prime}(0)}{\varepsilon}\right)^{\frac{1}{3}} e^{\frac{\pi}{6} i}$. Then $\psi_{\text {fast }, 0}$ solves the equation

$$
\left\{\begin{array}{l}
i \varepsilon \partial_{Y}^{2} \psi_{\text {fast }, 0}+Y U_{s}^{\prime}(0) \psi_{\text {fast }, 0}=0, \quad Y>0 \\
\left.\psi_{\text {fast }, 0}\right|_{Y=0}=\frac{1}{3^{\frac{2}{3}} \Gamma\left(\frac{2}{3}\right)}
\end{array}\right.
$$

Let $0<\varepsilon^{\frac{1}{3}} \alpha \leq 1$. Now we set

$$
\phi_{\text {app }, \text { fast }}(Y)=C_{\varepsilon, \alpha} \varepsilon^{-\frac{2}{3}} \int_{Y}^{\infty} e^{\alpha\left(Y-Y^{\prime}\right)} \int_{Y^{\prime}}^{\infty} e^{\alpha\left(Y^{\prime \prime}-Y^{\prime}\right)} \psi_{0, \text { fast }}\left(Y^{\prime \prime}\right) d Y^{\prime \prime} d Y^{\prime} .
$$

where the constant $C_{\varepsilon, \alpha}$ is chosen so that:

$$
\begin{aligned}
& \phi_{\text {app }, \text { fast }}(0)=1 \quad \text { if } \varepsilon^{-\frac{2}{3}}\left|\int_{0}^{\infty} e^{-\alpha Y^{\prime}} \int_{Y^{\prime}}^{\infty} e^{\alpha\left(Y^{\prime \prime}-Y^{\prime}\right)} \psi_{\text {fast }, 0}\left(Y^{\prime \prime}\right) d Y^{\prime \prime} d Y^{\prime}\right| \geq \frac{1}{10 U_{s}^{\prime}(0)^{\frac{2}{3}} 3^{\frac{1}{3}} \Gamma\left(\frac{1}{3}\right)} \\
& C_{\varepsilon, \alpha}=1 \quad \text { if } \varepsilon^{-\frac{2}{3}}\left|\int_{0}^{\infty} e^{-\alpha Y^{\prime}} \int_{Y^{\prime}}^{\infty} e^{\alpha\left(Y^{\prime \prime}-Y^{\prime}\right)} \psi_{\text {fast }, 0}\left(Y^{\prime \prime}\right) d Y^{\prime \prime} d Y^{\prime}\right|<\frac{1}{10 U_{s}^{\prime}(0)^{\frac{2}{3}} 3^{\frac{1}{3}} \Gamma\left(\frac{1}{3}\right)} .
\end{aligned}
$$

This choice of $C_{\varepsilon, \alpha}$ ensures the condition $\left|\phi_{\text {app }, \text { fast }}(0)\right| \leq 1+\frac{1}{10 U_{s}^{\prime}(0)^{\frac{2}{3}} 3^{\frac{1}{3}} \Gamma\left(\frac{1}{3}\right)}$.

By (7.79) and $0<\varepsilon^{\frac{1}{3}} \alpha \leq 1$ the integral defining $\phi_{\text {app,fast }}$ converges absolutely when $0<\varepsilon^{\frac{1}{3}} \alpha \leq 1$ and $\varepsilon>0$ is small enough. Note that we may assume the smallness of $\varepsilon$ by the condition $0<\varepsilon \leq \varepsilon_{1}$, where $\varepsilon_{1}>0$ is the number in Proposition 7.3 (by taking $\varepsilon_{1}$ even smaller if necessary). Note that $0<\left|C_{\varepsilon, \alpha}\right| \leq 1+10 U_{s}^{\prime}(0)^{\frac{2}{3}} 3^{\frac{1}{3}} \Gamma\left(\frac{1}{3}\right)$ by definition. Moreover, from the boundary layer structure of $\psi_{\text {fast }, 0}$ we can also show that

$$
1+10 U_{s}^{\prime}(0)^{\frac{2}{3}} 3^{\frac{1}{3}} \Gamma\left(\frac{1}{3}\right) \geq\left|C_{\varepsilon, \alpha}\right| \geq c>0,
$$

where $c$ is independent of $\varepsilon$ and $\alpha$ when $\varepsilon>0$ is small enough. We see that $\left(\partial_{Y}^{2}-\alpha^{2}\right) \phi_{\text {app }, \text { fast }}=$ $C_{\varepsilon, \alpha} \varepsilon^{-\frac{2}{3}} \psi_{\text {fast }, 0}$, and thus,

$$
O S\left[\phi_{a p p, f a s t}\right]=C_{\varepsilon, \alpha} \varepsilon^{-\frac{2}{3}}\left(U_{s}-U_{s}^{\prime}(0) Y\right) \psi_{f a s t, 0}-i \varepsilon \alpha^{2} C_{\varepsilon, \alpha} \varepsilon^{-\frac{2}{3}} \psi_{f a s t, 0}-U_{s}^{\prime \prime} \phi_{a p p, f a s t} .
$$

The fast mode $\phi_{\text {fast }}$ is then constructed in the form $\phi_{\text {fast }}=\phi_{\text {app }, \text { fast }}+\tilde{\phi}_{\text {fast }}$. To construct $\tilde{\phi}_{\text {fast }}$ we first solve

$$
\begin{aligned}
O S_{0}\left[\tilde{\phi}_{f a s t, 1}\right] & =-C_{\varepsilon, \alpha} \varepsilon^{-\frac{2}{3}}\left(U_{s}-U_{s}^{\prime}(0) Y\right) \psi_{\text {fast }, 0}+i C_{\varepsilon, \alpha} \varepsilon^{\frac{1}{3}} \alpha^{2} \psi_{f a s t, 0}+U_{s}^{\prime \prime} \phi_{\text {app }, \text { fast }}, \\
\left.\tilde{\phi}_{\text {fast }, 1}\right|_{Y=0} & =\left.\partial_{Y} \tilde{\phi}_{\text {fast }, 1}\right|_{Y=0}=0
\end{aligned}
$$


where $O S_{0}[\phi]=\partial_{Y}\left(U_{s} \partial_{Y} \phi\right)-\alpha^{2} U_{s} \phi+i \varepsilon\left(\partial_{Y}^{2}-\alpha^{2}\right)^{2} \phi$. Note that we have the relation $O S=$ $O S_{0}-\partial_{Y}\left(U_{s}^{\prime} \cdot\right)$. The advantage of introducing $O S_{0}$ is as follows:

(i) $O S_{0}$ has a good symmetry so that it is easy to solve by a simple energy method

(ii) the new error term $-\partial_{Y}\left(U_{s}^{\prime} \tilde{\phi}_{f a s t, 1}\right)$ has zero average and also vanishes on the boundary in virtue of the boundary condition imposed on $\tilde{\phi}_{\text {fast }, 1}$.

With this in mind let us set $\tilde{\phi}_{f a s t, 2}$ as the solution to (7.7) with the source term $f=$ $\partial_{Y}\left(U_{s}^{\prime} \tilde{\phi}_{f a s t, 1}\right)$, for which Corollary 7.5 can be applied. Then $\phi_{\text {fast }}=\phi_{\text {app }, \text { fast }}+\tilde{\phi}_{\text {fast }, 1}+\tilde{\phi}_{\text {fast }, 2}$ is our fast mode. The main result of this section is stated as follows.

Proposition 7.11. Let $\varepsilon_{1}>0$ be the number in Proposition 7.3. There exists a positive number $\delta_{1}$ such that if $0<\varepsilon \leq \varepsilon_{1}$ and $\varepsilon^{\frac{1}{3}} \alpha \leq \delta_{1}$ then there exists a function $\phi_{\text {fast }} \in H^{4}\left(\mathbb{R}_{+}\right)$ satisfying $O S\left[\phi_{\text {fast }}\right]=0$ and

$$
\begin{aligned}
\left\|\partial_{Y} \phi_{\text {fast }}\right\|+\alpha\left\|\phi_{\text {fast }}\right\| & \leq \frac{C}{\varepsilon^{\frac{1}{6}}}, \\
\left\|\left(\partial_{Y}^{2}-\alpha^{2}\right) \phi_{\text {fast }}\right\| & \leq \frac{C}{\varepsilon^{\frac{1}{2}}},
\end{aligned}
$$

and also

$$
\begin{aligned}
\phi_{\text {fast }}(0) & =1 \\
\partial_{Y} \phi_{\text {fast }}(0) & =\left(e^{\frac{\pi}{6} i} U_{s}^{\prime}(0)^{\frac{1}{3}} 3^{-\frac{2}{3}} \Gamma\left(\frac{1}{3}\right)+O\left(\varepsilon^{\frac{1}{3}} \alpha\right)+O\left(\varepsilon^{\frac{1}{3}}\right)\right) \varepsilon^{-\frac{1}{3}} .
\end{aligned}
$$

Proof. As stated above, we construct $\phi_{\text {fast }}$ of the form $\phi_{\text {fast }}=\phi_{\text {app }, \text { fast }}+\tilde{\phi}_{\text {fast }, 1}+\tilde{\phi}_{f a s t, 2}$, where $\phi_{a p p, f a s t}$ is given by

$$
\phi_{\text {app }, \text { fast }}=C_{\varepsilon, \alpha} \varepsilon^{-\frac{2}{3}} \int_{Y}^{\infty} e^{\alpha\left(Y-Y^{\prime}\right)} \int_{Y^{\prime}}^{\infty} e^{\alpha\left(Y^{\prime \prime}-Y^{\prime}\right)} \psi_{f a s t, 0}\left(Y^{\prime \prime}\right) d Y^{\prime \prime} d Y^{\prime} .
$$

From the definition of $\psi_{\text {fast }, 0}$ in (7.80) and the choice of $C_{\varepsilon, \alpha}$, it is straightforward to see

$$
\begin{aligned}
\left\|\partial_{Y} \phi_{\text {app }, \text { fast }}\right\| & \leq C \varepsilon^{-\frac{2}{3}}\left(\alpha \varepsilon^{\frac{2}{3}+\frac{1}{6}}+\varepsilon^{\frac{1}{3}+\frac{1}{6}}\right)=C\left(\varepsilon^{\frac{1}{6}} \alpha+\varepsilon^{-\frac{1}{6}}\right), \\
\alpha\left\|\phi_{\text {app }, \text { fast }}\right\| & \leq C \varepsilon^{-\frac{2}{3}} \alpha \cdot \varepsilon^{\frac{2}{3}+\frac{1}{6}}=C \alpha \varepsilon^{\frac{1}{6}}, \\
\left\|Y \phi_{\text {app }, \text { fast }}\right\| & \leq C \varepsilon^{\frac{1}{3}+\frac{1}{6}}=C \varepsilon^{\frac{1}{2}} \\
\left\|\left(\partial_{Y}^{2}-\alpha^{2}\right) \phi_{\text {app } . \text { fast }}\right\| & \leq C \varepsilon^{-\frac{2}{3}+\frac{1}{6}}=C \varepsilon^{-\frac{1}{2}},
\end{aligned}
$$

and

$$
\begin{aligned}
\partial_{Y} \phi_{\text {app }, f a s t}(0) & =C_{\varepsilon, \alpha} \varepsilon^{-\frac{2}{3}} \int_{0}^{\infty} e^{\alpha Y^{\prime \prime}} \psi_{0, f a s t}\left(Y^{\prime \prime}\right) d Y^{\prime \prime}+\alpha \phi_{\text {app }, \text { fast }}(0) \\
& =C_{\varepsilon, \alpha} \varepsilon^{-\frac{2}{3}} \int_{0}^{\infty} e^{\alpha Y^{\prime \prime}} \operatorname{Ai}\left(\lambda^{\frac{1}{3}} Y^{\prime \prime}\right) d Y^{\prime \prime}+O(\alpha) \\
& =C_{\varepsilon, \alpha} \varepsilon^{-\frac{2}{3}} \lambda^{-\frac{1}{3}} \int_{0}^{\infty} e^{\alpha \lambda^{-\frac{1}{3}} Z} \operatorname{Ai}(Z) d Z+O(\alpha) .
\end{aligned}
$$

Then let us recall that $\lambda^{\frac{1}{3}}=\left(\frac{U_{s}^{\prime}(0)}{\varepsilon}\right)^{\frac{1}{3}} e^{\frac{\pi}{6} i}$ and $\int_{0}^{\infty} \operatorname{Ai}(z) d z=\frac{1}{3}$. Hence, if $\varepsilon^{\frac{1}{3}} \alpha$ is small enough then the integral $\int_{0}^{\infty} e^{\alpha \lambda^{-\frac{1}{3}} Z} \operatorname{Ai}(Z) d Z$ is away from zero uniformly and we have a lower bound of the form

$$
\left|\partial_{Y} \phi_{a p p, f a s t}(0)\right| \geq \frac{1}{C \varepsilon^{\frac{1}{3}}}
$$


where $C>0$ is uniform in $\varepsilon$ and $\alpha$ as long as $\varepsilon^{\frac{1}{3}} \alpha$ is small enough. In fact, we can show the expansions as in (7.86) and (7.87) as follows. By using $e^{x}-1 \leq x e^{x}$ for $x \geq 0$ and the boundary layer structure of $\psi_{\text {fast }, 0}$, we have

$$
\begin{aligned}
\int_{0}^{\infty} e^{-\alpha Y^{\prime}} \int_{Y^{\prime}}^{\infty} e^{\alpha\left(Y^{\prime \prime}-Y^{\prime}\right)} \psi_{f a s t, 0}\left(Y^{\prime \prime}\right) d Y^{\prime \prime} d Y^{\prime} & =\int_{0}^{\infty} \int_{Y^{\prime}}^{\infty} \psi_{f a s t, 0}\left(Y^{\prime \prime}\right) d Y^{\prime \prime} d Y^{\prime}+O(\varepsilon \alpha) \\
& =\int_{0}^{\infty} Y \psi_{f a s t, 0}(Y) d Y+O(\varepsilon \alpha) \\
& =\int_{0}^{\infty} Y A i\left(\lambda^{\frac{1}{3}} Y\right) d Y+O(\varepsilon \alpha) \\
& =\lambda^{-\frac{2}{3}} \int_{0}^{\infty} Z A i(Z) d Z+O(\varepsilon \alpha) \\
& =\lambda^{-\frac{2}{3}} \int_{0}^{\infty} \partial_{Z}^{2} A i(Z) d Z+O(\varepsilon \alpha) \\
& =-\lambda^{-\frac{2}{3}} \partial_{Z} A i(0)+O(\varepsilon \alpha) \\
& =\varepsilon^{\frac{2}{3}} e^{-\frac{\pi}{3} i} \frac{1}{U_{s}^{\prime}(0)^{\frac{2}{3}}} \frac{1}{3^{\frac{1}{3}} \Gamma\left(\frac{1}{3}\right)}+O(\varepsilon \alpha) .
\end{aligned}
$$

Hence, if $0<\varepsilon^{\frac{1}{3}} \alpha \leq \delta_{1}$ is small enough, then the constant $C_{\varepsilon, \alpha}$ satisfies

$$
\begin{aligned}
C_{\varepsilon, \alpha} & =\left(\varepsilon^{-\frac{2}{3}} \int_{0}^{\infty} e^{\left.-\alpha Y^{\prime}\right)} \int_{Y^{\prime}}^{\infty} e^{\alpha\left(Y^{\prime \prime}-Y^{\prime}\right)} \psi_{\text {fast }, 0}\left(Y^{\prime \prime}\right) d Y^{\prime \prime} d Y^{\prime}\right)^{-1} \\
& =e^{\frac{\pi}{3} i} U_{s}^{\prime}(0)^{\frac{2}{3}} 3^{\frac{1}{3}} \Gamma\left(\frac{1}{3}\right)+O\left(\alpha \varepsilon^{\frac{1}{3}}\right)
\end{aligned}
$$

and thus we also have

$$
\phi_{\text {app }, \text { fast }}(0)=1
$$

and also

$$
\begin{aligned}
\partial_{Y} \phi_{\text {app }, f a s t}(0) & =C_{\varepsilon, \alpha} \varepsilon^{-\frac{2}{3}} \lambda^{-\frac{1}{3}}\left(\int_{0}^{\infty} A i(Z) d Z+O\left(\alpha \lambda^{-\frac{1}{3}}\right)\right)+O(\alpha) \\
& =\left(e^{\frac{\pi}{3} i} U_{s}^{\prime}(0)^{\frac{2}{3}} 3^{\frac{1}{3}} \Gamma\left(\frac{1}{3}\right)+O\left(\alpha \varepsilon^{\frac{1}{3}}\right)\right) \varepsilon^{-\frac{1}{3}} U_{s}^{\prime}(0)^{-\frac{1}{3}} e^{-\frac{\pi}{6} i}\left(\frac{1}{3}+O\left(\alpha \lambda^{-\frac{1}{3}}\right)\right)+O(\alpha) \\
& =\left(e^{\frac{\pi}{6} i} U_{s}^{\prime}(0)^{\frac{1}{3}} 3^{-\frac{2}{3}} \Gamma\left(\frac{1}{3}\right)+O\left(\alpha \varepsilon^{\frac{1}{3}}\right)\right) \varepsilon^{-\frac{1}{3}} .
\end{aligned}
$$

In particular, we have $\phi_{\text {fast }}(0)=1$, for $\phi_{\text {fast }}=\phi_{\text {app, fast }}+\tilde{\phi}_{\text {fast }}$ and $\tilde{\phi}_{\text {fast }}(0)=0$ by its construction. Next let us turn to the estimate of $\tilde{\phi}_{\text {fast }, 1}$. The idea is to apply Proposition 7.12 below, with $f_{2}=0$ and

$$
f_{1}=-\frac{\alpha}{i} \sigma\left[-C_{\varepsilon, \alpha} \varepsilon^{-\frac{2}{3}}\left(U_{s}-U_{s}^{\prime}(0) Y\right) \psi_{\text {fast }, 0}+i C_{\varepsilon, \alpha} \varepsilon^{\frac{1}{3}} \alpha^{2} \psi_{f a s t, 0}+U_{s}^{\prime \prime} \phi_{\text {app }, \text { fast }}\right]
$$

so that

$$
-\frac{i}{\alpha} \partial_{Y} f_{1}=-C_{\varepsilon, \alpha} \varepsilon^{-\frac{2}{3}}\left(U_{s}-U_{s}^{\prime}(0) Y\right) \psi_{f a s t, 0}+i C_{\varepsilon, \alpha} \varepsilon^{\frac{1}{3}} \alpha^{2} \psi_{f a s t, 0}+U_{s}^{\prime \prime} \phi_{\text {app }, \text { fast }} .
$$


Thus we have from Proposition 7.12 and also from Proposition 2.2 (1),

$$
\begin{aligned}
& \left\|\partial_{Y} \tilde{\phi}_{\text {fast }, 1}\right\|+\alpha\left\|\tilde{\phi}_{\text {fast }, 1}\right\| \\
& \leq \frac{C}{\epsilon^{\frac{1}{3}} \alpha}\left\|\frac{\alpha}{i} \sigma\left[-C_{\varepsilon, \alpha} \varepsilon^{-\frac{2}{3}}\left(U_{s}-U_{s}^{\prime}(0) Y\right) \psi_{\text {fast }, 0}+i C_{\varepsilon, \alpha} \varepsilon^{\frac{1}{3}} \alpha^{2} \psi_{\text {fast }, 0}+U_{s}^{\prime \prime} \phi_{\text {app }, \text { fast }}\right]\right\| \\
& \leq \frac{C}{\epsilon^{\frac{1}{3}}}\left\|Y\left(-C_{\varepsilon, \alpha} \varepsilon^{-\frac{2}{3}}\left(U_{s}-U_{s}^{\prime}(0) Y\right) \psi_{\text {fast }, 0}+i C_{\varepsilon, \alpha} \varepsilon^{\frac{1}{3}} \alpha^{2} \psi_{\text {fast }, 0}+U_{s}^{\prime \prime} \phi_{\text {app }, \text { fast }}\right)\right\| \\
& \leq \frac{C}{\varepsilon^{\frac{1}{3}}}\left(\varepsilon^{-\frac{2}{3}}\left\|Y^{3} \psi_{\text {fast }, 0}\right\|+\varepsilon^{\frac{1}{3}} \alpha^{2}\left\|Y \psi_{\text {fast }, 0}\right\|+\left\|Y \phi_{\text {app }, \text { fast }}\right\|\right) .
\end{aligned}
$$

Hence, the boundary layer structure of $\psi_{\text {fast }, 0}$ and $\phi_{a p p, f a s t}$ (e.g., see (7.89)) implies

$$
\left\|\partial_{Y} \tilde{\phi}_{f a s t, 1}\right\|+\alpha\left\|\tilde{\phi}_{\text {fast }, 1}\right\| \leq C \varepsilon^{\frac{1}{6}}+C \varepsilon^{\frac{1}{2}} \alpha^{2} .
$$

Similarly, we have the $H^{2}$ bound such as

$$
\left\|\left(\partial_{Y}^{2}-\alpha^{2}\right) \tilde{\phi}_{f a s t, 1}\right\| \leq \frac{C}{\varepsilon^{\frac{1}{3}}}\left(\varepsilon^{\frac{1}{6}}+\varepsilon^{\frac{1}{2}} \alpha^{2}\right) .
$$

Finally let us estimate $\tilde{\phi}_{f a s t, 2}$, which is the solution to $(7.7)$ with the source term $f=$ $\partial_{Y}\left(U_{s}^{\prime} \tilde{\phi}_{\text {fast }, 1}\right)$. Corollary 7.5 implies that

$$
\left\|\partial_{Y} \tilde{\phi}_{f a s t, 2}\right\|+\alpha\left\|\tilde{\phi}_{f a s t, 2}\right\| \leq C\left\|(1+Y)^{2} \partial_{Y}\left(U_{s}^{\prime} \tilde{\phi}_{f a s t, 1}\right)\right\| \leq C\left\|\partial_{Y} \tilde{\phi}_{f a s t, 1}\right\| \leq C \varepsilon^{\frac{1}{6}}+C \varepsilon^{\frac{1}{2}} \alpha^{2},
$$

and

$$
\begin{aligned}
\left\|\left(\partial_{Y}^{2}-\alpha^{2}\right) \tilde{\phi}_{f a s t, 2}\right\| & \leq C\left\|(1+Y)^{2} \partial_{Y}\left(U_{s}^{\prime} \tilde{\phi}_{f a s t, 1}\right)\right\|+C\left\|\frac{\partial_{Y}\left(U_{s}^{\prime} \tilde{\phi}_{f a s t, 1}\right)}{U_{s}}\right\| \\
& \leq C\left\|\partial_{Y} \tilde{\phi}_{f a s t, 1}\right\|+C\left\|\partial_{Y}^{2} \tilde{\phi}_{f a s t, 1}\right\| \\
& \leq C \\
\varepsilon^{\frac{1}{3}} & \left(\varepsilon^{\frac{1}{6}}+\varepsilon^{\frac{1}{2}} \alpha^{2}\right) .
\end{aligned}
$$

Collecting (7.93), (7.94), and (7.96), we have for $\tilde{\phi}_{f a s t}=\tilde{\phi}_{f a s t, 1}+\tilde{\phi}_{f a s t, 2}$,

$$
\begin{aligned}
\left\|\partial_{Y} \tilde{\phi}_{\text {fast }}\right\|+\alpha\left\|\tilde{\phi}_{\text {fast }}\right\| & \leq C \varepsilon^{\frac{1}{6}}+C \varepsilon^{\frac{1}{2}} \alpha^{2}, \\
\left\|\left(\partial_{Y}^{2}-\alpha^{2}\right) \tilde{\phi}_{\text {fast }}\right\| & \leq \frac{C}{\varepsilon^{\frac{1}{3}}}\left(\varepsilon^{\frac{1}{6}}+\varepsilon^{\frac{1}{2}} \alpha^{2}\right), \\
\left\|\partial_{Y} \tilde{\phi}_{\text {fast }}\right\|_{L^{\infty}} & \leq \frac{C}{\varepsilon^{\frac{1}{6}}}\left(\varepsilon^{\frac{1}{6}}+\varepsilon^{\frac{1}{2}} \alpha^{2}\right) \leq C\left(1+\varepsilon^{\frac{1}{3}} \alpha^{2}\right) .
\end{aligned}
$$

Then $\phi_{\text {fast }}=\phi_{\text {app,fast }}+\tilde{\phi}_{\text {fast }}$ satisfies $(7.84)$ and (7.85) when $0<\varepsilon^{\frac{1}{3}} \alpha \leq \delta_{1}$, and the expansions (7.86) and (7.87) follow from (7.91), (7.92), and (7.97). The proof is complete.

\subsection{Proof of Theorem 7.1 for $\varepsilon^{\frac{1}{3}} \alpha \ll 1$}

In this subsection we prove Theorem 7.1 when $\varepsilon^{\frac{1}{3}} \alpha \ll 1$ and $0<\varepsilon \leq \varepsilon_{1} \ll 1$. As explained in the beginning of Subsection 7.2, we construct the solution $\phi$ to (7.1) of the form

$$
\phi=\Phi_{\text {slip }}[f]+a \phi_{\text {slow }}+b \phi_{\text {fast }},
$$


where $\Phi_{\text {slip }}[f]$ is the solution to the modified Orr-Sommerfeld equation (7.7) obtained in Proposition $7.3, \phi_{\text {slow }}$ and $\phi_{\text {fast }}$ are respectively the slow mode and the fast mode constructed in Propositions 7.9, 7.10, and 7.11. The coefficients $a$ and $b$ have to be chosen so that

$$
\tilde{\phi}=a \phi_{\text {slow }}+b \phi_{\text {fast }}
$$

satisfies the boundary condition $\left.\tilde{\phi}\right|_{Y=0}=0,\left.\partial_{Y} \tilde{\phi}\right|_{Y=0}=-\left.\partial_{Y} \Phi_{\text {slip }}[f]\right|_{Y=0}$, which is equivalent with the linear relation

$$
M\left(\begin{array}{l}
a \\
b
\end{array}\right)=\left(\begin{array}{c}
0 \\
-\left.\partial_{Y} \Phi_{\text {slip }}[f]\right|_{Y=0}
\end{array}\right), \quad M=\left(\begin{array}{cc}
\left.\phi_{\text {slow }}\right|_{Y=0} & \left.\phi_{\text {fast }}\right|_{Y=0} \\
\left.\partial_{Y} \phi_{\text {slow }}\right|_{Y=0} & \left.\partial_{Y} \phi_{\text {fast }}\right|_{Y=0}
\end{array}\right) .
$$

Hence we have

$$
\begin{aligned}
\left(\begin{array}{l}
a \\
b
\end{array}\right) & =\frac{1}{\operatorname{det} M}\left(\begin{array}{cc}
\left.\partial_{Y} \phi_{\text {fast }}\right|_{Y=0} & -\left.\phi_{\text {fast }}\right|_{Y=0} \\
-\left.\partial_{Y} \phi_{\text {slow }}\right|_{Y=0} & \left.\phi_{\text {slow }}\right|_{Y=0}
\end{array}\right)\left(\begin{array}{c}
0 \\
-\left.\partial_{Y} \Phi_{\text {slip }}[f]\right|_{Y=0}
\end{array}\right) \\
& =\frac{\left.\partial_{Y} \Phi_{\text {slip }}[f]\right|_{Y=0}}{\operatorname{det} M}\left(\begin{array}{c}
\left.\phi_{\text {fast }}\right|_{Y=0} \\
-\left.\phi_{\text {slow }}\right|_{Y=0}
\end{array}\right), \quad \operatorname{det} M=\left.\left(\phi_{\text {slow }} \partial_{Y} \phi_{\text {fast }}-\phi_{\text {fast }} \partial_{Y} \phi_{\text {slow }}\right)\right|_{Y=0} .
\end{aligned}
$$

From Propositions 7.10 and 7.11 we observe that

$$
|\operatorname{det} M| \geq \frac{1}{C \varepsilon^{\frac{1}{3}}}-C\left(\frac{1}{\varepsilon^{\frac{1}{6}}}+\alpha\right) \geq \frac{1}{2 C \varepsilon^{\frac{1}{3}}} \quad \text { if } \varepsilon^{\frac{1}{3}} \alpha \ll 1 \text { and } \alpha \geq 1 .
$$

Hence, when $0<\varepsilon^{\frac{1}{3}} \alpha \ll 1$ and $\alpha \geq 1$ it is easy to see from Proposition 7.10 and Proposition 7.11

$$
\begin{aligned}
\left\|\partial_{Y} \tilde{\phi}\right\|+\alpha\|\tilde{\phi}\| & \leq C \varepsilon^{\frac{1}{3}}\left|\partial_{Y} \Phi_{\text {slip }}[f]\right|_{Y=0} \mid\left(\left\|\partial_{Y} \phi_{\text {slow }}\right\|+\alpha\left\|\phi_{\text {slow }}\right\|+\left\|\partial_{Y} \phi_{\text {fast }}\right\|+\alpha\left\|\phi_{\text {fast }}\right\|\right) \\
& \leq C \varepsilon^{\frac{1}{3}}\left|\partial_{Y} \Phi_{\text {slip }}[f]\right|_{Y=0} \mid\left(\alpha^{\frac{1}{2}}+\frac{1}{\varepsilon^{\frac{1}{6}}}\right) \\
& \leq C \varepsilon^{\frac{1}{6}}\left|\partial_{Y} \Phi_{\text {slip }}[f]\right|_{Y=0} \mid
\end{aligned}
$$

and similarly,

$$
\begin{aligned}
\left\|\left(\partial_{Y}^{2}-\alpha^{2}\right) \tilde{\phi}\right\| & \leq C \varepsilon^{\frac{1}{3}}\left|\partial_{Y} \Phi_{\text {slip }}[f]\right|_{Y=0} \mid\left(\left\|\left(\partial_{Y}^{2}-\alpha^{2}\right) \phi_{\text {slow }}\right\|+\left\|\left(\partial_{Y}^{2}-\alpha^{2}\right) \phi_{\text {fast }}\right\|\right) \\
& \leq C \varepsilon^{\frac{1}{3}}\left|\partial_{Y} \Phi_{\text {slip }}[f]\right|_{Y=0} \mid\left(\frac{1}{\varepsilon^{\frac{1}{3}}}+\frac{1}{\varepsilon^{\frac{1}{2}}}\right) \\
& \leq \frac{C}{\varepsilon^{\frac{1}{6}}}\left|\partial_{Y} \Phi_{\text {slip }}[f]\right|_{Y=0} \mid .
\end{aligned}
$$

Combining the last two bounds with (7.52), we deduce the existence of a solution satisfying (7.2)-(7.3). If $0<\alpha \leq 1$ we use the asymptotic estimates (7.67), (7.71), (7.86), and (7.87), which yield

$$
\begin{aligned}
\operatorname{det} M & =\left(e^{\frac{\pi}{6} i} U_{s}^{\prime}(0)^{\frac{1}{3}} 3^{-\frac{2}{3}} \Gamma\left(\frac{1}{3}\right)+O\left(\varepsilon^{\frac{1}{3}} \alpha\right)+O\left(\varepsilon^{\frac{1}{3}}\right)\right) \varepsilon^{-\frac{1}{3}}-\frac{c_{E} U_{s}^{\prime}(0)}{\alpha}+O\left(\frac{\varepsilon^{\frac{1}{12}}}{\alpha}+\frac{1}{\varepsilon^{\frac{1}{4}}}\right) \\
& =\left(e^{\frac{\pi}{6} i} U_{s}^{\prime}(0)^{\frac{1}{3}} 3^{-\frac{2}{3}} \Gamma\left(\frac{1}{3}\right) \frac{1}{\varepsilon^{\frac{1}{3}}}-\frac{c_{E} U_{s}^{\prime}(0)}{\alpha}\right)(1+o(1)),
\end{aligned}
$$

in the case $0<\varepsilon \leq \varepsilon_{1} \ll 1$. Hence we have the lower bound

$$
|\operatorname{det} M| \geq \frac{1}{C}\left(\frac{1}{\alpha}+\frac{1}{\varepsilon^{\frac{1}{3}}}\right) .
$$


Thus, we have for $0<\varepsilon^{\frac{1}{3}} \alpha \ll 1$ and $0<\alpha \leq 1$,

$$
\begin{aligned}
\left\|\partial_{Y} \tilde{\phi}\right\|+\alpha\|\tilde{\phi}\| & \leq C \frac{\varepsilon^{\frac{1}{3}} \alpha}{\alpha+\varepsilon^{\frac{1}{3}}}\left|\partial_{Y} \Phi_{\text {slip }}[f]\right|_{Y=0} \mid\left(\left\|\partial_{Y} \phi_{\text {slow }}\right\|+\alpha\left\|\phi_{\text {slow }}\right\|+\left\|\partial_{Y} \phi_{\text {fast }}\right\|+\alpha\left\|\phi_{\text {fast }}\right\|\right) \\
& \leq C \frac{\varepsilon^{\frac{1}{3}} \alpha}{\alpha+\varepsilon^{\frac{1}{3}}}\left|\partial_{Y} \Phi_{\text {slip }}[f]\right|_{Y=0} \mid\left(\frac{1}{\alpha}+\frac{1}{\varepsilon^{\frac{1}{6}}}\right) .
\end{aligned}
$$

Similarly, using (7.69), (7.84)-(7.85), we find

$$
\begin{aligned}
\left\|\partial_{Y} \tilde{\phi}\right\|_{L^{\infty}} & \leq C \frac{\varepsilon^{\frac{1}{3}} \alpha}{\alpha+\varepsilon^{\frac{1}{3}}}\left|\partial_{Y} \Phi_{\text {slip }}[f]\right|_{Y=0} \mid\left(\left\|\partial_{Y} \phi_{\text {slow }}\right\|_{L^{\infty}}+\left\|\partial_{Y} \phi_{\text {fast }}\right\|_{L^{\infty}}\right) \\
& \leq C \frac{\varepsilon^{\frac{1}{3}} \alpha}{\alpha+\varepsilon^{\frac{1}{3}}}\left(\frac{1}{\alpha}+C\left(\frac{\varepsilon^{\frac{1}{12}}}{\alpha}+\frac{1}{\varepsilon^{\frac{1}{4}}}\right)+\frac{1}{\varepsilon^{\frac{1}{3}}}\right) \\
& \leq C\left|\partial_{Y} \Phi_{\text {slip }}[f]\right|_{Y=0} \mid .
\end{aligned}
$$

As for the $H^{2}$ estimate, the similar argument shows

$$
\begin{aligned}
\left\|\left(\partial_{Y}^{2}-\alpha^{2}\right) \tilde{\phi}\right\| & \leq C \frac{\varepsilon^{\frac{1}{3}} \alpha}{\alpha+\varepsilon^{\frac{1}{3}}}\left|\partial_{Y} \Phi_{\text {slip }}[f]\right|_{Y=0} \mid\left(\left\|\left(\partial_{Y}^{2}-\alpha^{2}\right) \phi_{\text {slow }}\right\|+\left\|\left(\partial_{Y}^{2}-\alpha^{2}\right) \phi_{\text {fast }}\right\|\right) \\
& \leq C \frac{\varepsilon^{\frac{1}{3}} \alpha}{\alpha+\varepsilon^{\frac{1}{3}}}\left|\partial_{Y} \Phi_{\text {slip }}[f]\right|_{Y=0} \mid\left(\frac{1}{\alpha}+\frac{1}{\varepsilon^{\frac{1}{6}} \alpha}+\frac{1}{\varepsilon^{\frac{1}{3}}}+\frac{1}{\varepsilon^{\frac{1}{2}}}\right) \\
& \leq C \frac{\varepsilon^{\frac{1}{6}} \alpha}{\alpha+\varepsilon^{\frac{1}{3}}}\left|\partial_{Y} \Phi_{\text {slip }}[f]\right|_{Y=0} \mid\left(\frac{1}{\alpha}+\frac{1}{\varepsilon^{\frac{1}{3}}}\right) .
\end{aligned}
$$

Combining with (7.53), we recover the existence of a solution with the bounds (7.4)-(7.5)-(7.6).

The uniqueness of the solution follows from the theory of ordinary differential equations. Indeed, there exist four linearly independent solutions to the fourth order differential equation $O S[\phi]=0$, and two of which are taken as the slow mode and the fast mode constructed as above, while the other two grow as $Y \rightarrow \infty$. Hence, if $\phi$ is the solution to $O S[\phi]=0$ decaying as $Y \rightarrow \infty$ with $\left.\phi\right|_{Y=0}=\left.\partial_{Y} \phi\right|_{Y=0}=0$, then $\phi$ must be a linear combination of $\phi_{\text {slow }}$ and $\phi_{\text {fast }}$, and then, as we have seen that $\operatorname{det} M \neq 0, \phi$ must be trivial. The proof of Theorem 7.1 is complete.

\subsection{Proof of Theorem 4.1 for $|\tilde{n}| \leq \delta \nu^{-\frac{3}{4}}$}

In this section we prove Theorem 4.1 for frequencies $\tilde{n}$ satisfying $0<|\tilde{n}| \nu^{\frac{3}{4}} \ll 1$. We may assume that $\tilde{n}>0$ and note that $0<\tilde{n} \nu^{\frac{3}{4}} \ll 1$ corresponds to $0<\varepsilon^{\frac{1}{3}} \alpha \ll 1$. We also assume that the source term $f_{n}$ belongs to $H^{1}\left(\mathbb{R}_{+}\right)^{2}$ : the existence result for the case $f_{n} \in L^{2}\left(\mathbb{R}_{+}\right)^{2}$ follows from our uniform estimate and a standard density argument. With this in mind, we consider the Orr-Sommerfeld equations (4.12) with $f \in H^{1}\left(\mathbb{R}_{+}\right)^{2}$. These equations are expressed in the rescaled variable $Y$, and are equivalent to the original problem (1.13). An important point here is that the source term $-f_{2}-\frac{i}{\alpha} \partial_{Y} f_{1}$ is not compatible with a direct application of Theorem 7.1. To fill this discrepancy we observe the identity

$$
\begin{aligned}
O S[\phi]= & \partial_{Y}\left(U_{s} \partial_{Y} \phi\right)-\alpha^{2} U_{s} \phi-U_{s}^{\prime} \partial_{Y} \phi-U_{s}^{\prime \prime} \phi+i \varepsilon\left(\partial_{Y}^{2}-\alpha^{2}\right)^{2} \phi \\
= & \partial_{Y}\left(U_{s} \partial_{Y} \phi\right)-\alpha^{2} U_{s} \phi+i \varepsilon\left(\partial_{Y}^{2}-\alpha^{2}\right)^{2} \phi \\
& -U_{s}^{\prime} \partial_{Y} \phi-U_{s}^{\prime \prime} \phi \\
= & : O S_{0}[\phi]-U_{s}^{\prime} \partial_{Y} \phi-U_{s}^{\prime \prime} \phi
\end{aligned}
$$


and then we first consider the problem

$$
\left\{\begin{array}{l}
O S_{0}\left[\phi_{0}\right]=-f_{2}-\frac{i}{\alpha} \partial_{Y} f_{1}, \quad Y>0, \\
\left.\phi_{0}\right|_{Y=0}=\left.\partial_{Y} \phi_{0}\right|_{Y=0}=0 .
\end{array}\right.
$$

Proposition 7.12. Let $\alpha>0$. Then for any $f=\left(f_{1}, f_{2}\right) \in H^{1}\left(\mathbb{R}_{+}\right)^{2}$ there exists a unique solution $\phi_{0} \in H_{0}^{2}\left(\mathbb{R}_{+}\right) \cap H^{4}\left(\mathbb{R}_{+}\right)$to (7.106) such that

$$
\begin{aligned}
\left\|\partial_{Y} \phi_{0}\right\|+\alpha\left\|\phi_{0}\right\| & \leq \frac{C}{\varepsilon^{\frac{1}{3}} \alpha}\|f\|, \\
\left\|\left(\partial_{Y}^{2}-\alpha^{2}\right) \phi_{0}\right\| & \leq \frac{C}{\varepsilon^{\frac{2}{3}} \alpha}\|f\| .
\end{aligned}
$$

Proof. We focus on the a priori estimate. For simplicity of notations we set

$$
E=\left\|\partial_{Y} \phi_{0}\right\|^{2}+\alpha^{2}\left\|\phi_{0}\right\|^{2}
$$

By taking the inner product with $\phi_{0}$ in the equation (7.106) we obtain

$$
\left\|\sqrt{U_{s}} \partial_{Y} \phi_{0}\right\|^{2}+\alpha^{2}\left\|\sqrt{U_{s}} \phi_{0}\right\|^{2}-i \varepsilon\left\|\left(\partial_{Y}^{2}-\alpha^{2}\right) \phi_{0}\right\|^{2}=\left\langle f_{2}+\frac{i}{\alpha} \partial_{Y} f_{1}, \phi_{0}\right\rangle,
$$

and thus, the real part and the imaginary part of this identity give

$$
\left\|\sqrt{U_{s}} \partial_{Y} \phi_{0}\right\|^{2}+\alpha^{2}\left\|\sqrt{U_{s}} \phi_{0}\right\|^{2}=\operatorname{Re}\left\langle f_{2}+\frac{i}{\alpha} \partial_{Y} f_{1}, \phi_{0}\right\rangle,
$$

and

$$
\varepsilon\left\|\left(\partial_{Y}^{2}-\alpha^{2}\right) \phi_{0}\right\|^{2}=-\mathcal{I} m\left\langle f_{2}+\frac{i}{\alpha} \partial_{Y} f_{1}, \phi_{0}\right\rangle .
$$

Equality (7.109) implies

$$
\left\|\sqrt{U_{s}} \partial_{Y} \phi_{0}\right\|^{2}+\alpha^{2}\left\|\sqrt{U_{s}} \phi_{0}\right\|^{2} \leq \frac{2}{\alpha}\|f\| E^{\frac{1}{2}} .
$$

On the other hand, we have from (7.110),

$$
\varepsilon\left\|\left(\partial_{Y}^{2}-\alpha^{2}\right) \phi_{0}\right\|^{2} \leq 2 \frac{\|f\|}{\alpha} E^{\frac{1}{2}}
$$

By using the interpolation inequality we have

$$
\begin{aligned}
E \leq & C\left\|\sqrt{U_{s}} \partial_{Y} \phi_{0}\right\|^{\frac{4}{3}}\left\|\partial_{Y}^{2} \phi_{0}\right\|^{\frac{2}{3}}+C\left\|\sqrt{U_{s}} \partial_{Y} \phi_{0}\right\|^{2} \\
& +C \alpha^{2}\left\|\sqrt{U_{s}} \phi_{0}\right\|^{\frac{4}{3}}\left\|\partial_{Y} \phi_{0}\right\|^{\frac{2}{3}}+C \alpha^{2}\left\|\sqrt{U_{s}} \phi_{0}\right\|^{2} \\
\leq & C\left(\frac{\|f\| E^{\frac{1}{2}}}{\alpha}\right)^{\frac{2}{3}}\left(\frac{1}{\varepsilon} \frac{\|f\|}{\alpha} E^{\frac{1}{2}}\right)^{\frac{1}{3}}+C \alpha^{\frac{2}{3}}\left(\frac{\|f\| E^{\frac{1}{2}}}{\alpha}\right)^{\frac{2}{3}} E^{\frac{1}{3}}+C \frac{\|f\|}{\alpha} E^{\frac{1}{2}} \\
\leq & \frac{C}{\varepsilon^{\frac{1}{3}} \alpha}\|f\| E^{\frac{1}{2}}+C\|f\|^{\frac{2}{3}} E^{\frac{2}{3}}+C \frac{\|f\|}{\alpha} E^{\frac{1}{2}} .
\end{aligned}
$$

This implies

$$
E \leq C\left(\frac{1}{\varepsilon^{\frac{2}{3}} \alpha^{2}}+1\right)\|f\|^{2} \leq \frac{C}{\varepsilon^{\frac{2}{3}} \alpha^{2}}\|f\|^{2} .
$$


Here we have used $0<\tilde{n} \leq \nu^{-\frac{3}{4}}$. Estimate (7.107) is proved. Then we have from (7.112),

$$
\left\|\left(\partial_{Y}^{2}-\alpha^{2}\right) \phi_{0}\right\|^{2} \leq \frac{C}{\varepsilon \alpha} \frac{1}{\varepsilon^{\frac{1}{3}} \alpha}\|f\|^{2} \leq \frac{C}{\varepsilon^{\frac{4}{3}} \alpha^{2}}\|f\|^{2},
$$

which shows (7.108). We have proved the a priori estimates and the uniqueness. The regularity $\phi_{0} \in H^{4}\left(\mathbb{R}_{+}\right)$follows from the elliptic regularity. As for the existence, we introduce the operator $O S_{0, l}=O S_{0}+i l$ for a constant $l>0$, and then it is easy to see that $O S_{0, l}$ (under the same boundary condition as above) is invertible if $l$ is sufficiently large. The argument above holds exactly in the same way even for $O S_{0, l}$ with $l>0$, and hence, the estimate is uniform in $l>0$. Then we can prove the existence of the solution for $l=0$ by the standard continuity method about $l$. The details are omitted here. The proof is complete.

Let us prove Theorem 4.1. As mentioned in the beginning of this subsection, we first assume $f_{n} \in H^{1}\left(\mathbb{R}_{+}\right)^{2}$. The solution $\phi \in H^{4}\left(\mathbb{R}_{+}\right)$to (4.12) is constructed in the form $\phi=\phi_{0}+\phi_{1}$, where $\phi_{0}$ is the solution to (7.106) obtained in Proposition 7.12, and $\phi_{1}$ is the solution to (7.1) with $f$ replaced by $U_{s}^{\prime} \partial_{Y} \phi_{0}+U_{s}^{\prime \prime} \phi_{0}=\partial_{Y}\left(U_{s}^{\prime} \phi_{0}\right)$, for which Theorem 7.1 is applied without loss of $\alpha^{-1}$ when $\alpha$ is small, in virtue of the fact $\int_{0}^{\infty} \partial_{Y}\left(U_{s}^{\prime} \phi_{0}\right) d Y=0$. Note that, in order to apply Theorem 7.1, we need the condition $0<\varepsilon^{\frac{1}{3}} \alpha \leq \delta_{0}$, which is equivalent with $0<\tilde{n} \leq \delta_{0}^{\frac{3}{2}} \nu^{-\frac{3}{4}}$. Let $\alpha \geq 1$ in addition, which is the case $\tilde{n} \geq \nu^{-\frac{1}{2}}$. Theorem 7.1 implies that

$$
\begin{aligned}
\left\|\partial_{Y} \phi_{1}\right\|+\alpha\left\|\phi_{1}\right\| & \leq C\left\|(1+Y) \partial_{Y}\left(U_{s}^{\prime} \phi_{0}\right)\right\| \leq C\left\|\partial_{Y} \phi_{0}\right\|, \\
\left\|\left(\partial_{Y}^{2}-\alpha^{2}\right) \phi_{1}\right\| & \leq \frac{C}{\varepsilon^{\frac{1}{3}}}\left\|(1+Y) \partial_{Y}\left(U_{s}^{\prime} \phi_{0}\right)\right\| \leq \frac{C}{\varepsilon^{\frac{1}{3}}}\left\|\partial_{Y} \phi_{0}\right\| .
\end{aligned}
$$

Therefore, Proposition 7.12 and (7.115)-(7.116) yield for $\phi=\phi_{0}+\phi_{1}$,

$$
\begin{aligned}
\left\|\partial_{Y} \phi\right\|+\alpha\|\phi\| & \leq \frac{C}{\varepsilon^{\frac{1}{3}} \alpha}\|f\|, \\
\left\|\left(\partial_{Y}^{2}-\alpha^{2}\right) \phi\right\| & \leq \frac{C}{\varepsilon^{\frac{2}{3}} \alpha}\|f\| .
\end{aligned}
$$

Rescaling back to the original variable and recalling (4.11), we have from (7.117),

$$
\left\|u_{n}\right\|_{L^{2}} \leq \frac{C}{\varepsilon^{\frac{1}{3}} \tilde{n}}\left\|f_{n}\right\|_{L^{2}} \leq \frac{C}{\tilde{n}^{\frac{2}{3}}}\left\|f_{n}\right\|_{L^{2}}
$$

and from (7.118),

$$
\left\|\partial_{y} u_{n}\right\|_{L^{2}}+\tilde{n}\left\|u_{n}\right\|_{L^{2}} \leq \frac{C}{\tilde{n}^{\frac{1}{3}} \nu^{\frac{1}{2}}}\left\|f_{n}\right\|_{L^{2}}
$$

This proves (4.4) and (4.5) in the case $0<|\tilde{n}| \leq \delta_{0}^{\frac{3}{2}} \nu^{-\frac{3}{4}}$ and $\tilde{n} \geq \nu^{-\frac{1}{2}}$, where $\delta_{0}>0$ is the number in Theorem 7.1. Next we consider the case $0<\alpha \leq 1$, that is, $\tilde{n} \leq \nu^{-\frac{1}{2}}$. In this case Theorem 7.1 shows that

$$
\begin{aligned}
\left\|\partial_{Y} \phi_{1}\right\|+\alpha\left\|\phi_{1}\right\| & \leq C \frac{\alpha+\varepsilon^{\frac{1}{6}}}{\alpha+\varepsilon^{\frac{1}{3}}}\left\|(1+Y)^{2} \partial_{Y}\left(U_{s}^{\prime} \phi_{0}\right)\right\| \leq C \frac{\alpha+\varepsilon^{\frac{1}{6}}}{\alpha+\varepsilon^{\frac{1}{3}}}\left\|\partial_{Y} \phi_{0}\right\|, \\
\left\|\partial_{Y} \phi_{1}\right\|_{L^{\infty}}+\alpha\left\|\phi_{1}\right\|_{L^{\infty}} & \leq \frac{C}{\varepsilon^{\frac{1}{6}}}\left\|(1+Y)^{2} \partial_{Y}\left(U_{s}^{\prime} \phi_{0}\right)\right\| \leq \frac{C}{\varepsilon^{\frac{1}{6}}}\left\|\partial_{Y} \phi_{0}\right\| \\
\left\|\left(\partial_{Y}^{2}-\alpha^{2}\right) \phi_{1}\right\| & \leq \frac{C}{\varepsilon^{\frac{1}{3}}}\left\|(1+Y)^{2} \partial_{Y}\left(U_{s}^{\prime} \phi_{0}\right)\right\| \leq \frac{C}{\varepsilon^{\frac{1}{3}}}\left\|\partial_{Y} \phi_{0}\right\|
\end{aligned}
$$


Hence, combining with Proposition 7.12, we obtain

$$
\begin{aligned}
\left\|\partial_{Y} \phi\right\|+\alpha\|\phi\| & \leq C \frac{\alpha+\varepsilon^{\frac{1}{6}}}{\left(\alpha+\varepsilon^{\frac{1}{3}}\right) \varepsilon^{\frac{1}{3}} \alpha}\|f\|, \\
\left\|\partial_{Y} \phi\right\|_{L^{\infty}}+\alpha\|\phi\|_{L^{\infty}} & \leq \frac{C}{\varepsilon^{\frac{1}{2}} \alpha}\|f\|, \\
\left\|\left(\partial_{Y}^{2}-\alpha^{2}\right) \phi\right\| & \leq \frac{C}{\varepsilon^{\frac{2}{3}} \alpha}\|f\| .
\end{aligned}
$$

In the original variable these estimates provide

$$
\begin{aligned}
\left\|u_{n}\right\|_{L^{2}} & \leq C \frac{\tilde{n} \nu^{\frac{1}{2}}+\tilde{n}^{-\frac{1}{6}}}{\left(\tilde{n} \nu^{\frac{1}{2}}+\tilde{n}^{-\frac{1}{3}}\right) \tilde{n}^{\frac{2}{3}}}\left\|f_{n}\right\|_{L^{2}}, \\
\left\|u_{n}\right\|_{L^{\infty}} & \leq \frac{C}{\tilde{n}^{\frac{1}{2}} \nu^{\frac{1}{4}}}\left\|f_{n}\right\|_{L^{2}}, \\
\left\|\partial_{y} u_{n}\right\|_{L^{2}}+\tilde{n}\left\|u_{n}\right\|_{L^{2}} & \leq \frac{C}{\tilde{n}^{\frac{1}{3}} \nu^{\frac{1}{2}}}\left\|f_{n}\right\|_{L^{2}} .
\end{aligned}
$$

This proves (4.1), (4.2), and (4.3), by comparing the size of $\tilde{n} \nu^{\frac{1}{2}}$ and of $\tilde{n}^{-\frac{1}{6}}$ or $\tilde{n}^{-\frac{1}{3}}$. In virtue of the assumption $f_{n} \in H^{1}\left(\mathbb{R}_{+}\right)^{2}$ we have the $H^{3}$ regularity of $u_{n}$. The bound of the $H^{2}$ norm of $u_{n}$ in terms of the $L^{2}$ norm of $f_{n}$ is then recovered from the elliptic regularity of the Stokes operator, for the $L^{2}$ norm of the term $-i \tilde{n} U_{s}^{\nu} u_{n}-u_{n, 2} \partial_{y} U_{s}^{\nu} \mathbf{e}_{1}+f_{n}$ in (1.13) is bounded in terms of $\left\|f_{n}\right\|_{L^{2}}$ as already proved above. The details are omitted here. Hence, the existence and the estimates of the solution $u_{n}$ for the case $f_{n} \in L^{2}\left(\mathbb{R}_{+}\right)^{2}$ follow from a standard density argument. As for the uniqueness, if $u_{n} \in H^{2}\left(\mathbb{R}_{+}\right)^{2} \cap H_{0}^{1}\left(\mathbb{R}_{+}\right)^{2}$ is the solution to (1.13) with $f_{n}=0$, then $u_{n}$ is smooth and the associated streamfunction $\phi$ in the rescaled variable satisfies (7.1) with $f=0$. Hence Theorem 7.1 implies $\phi=0$, and thus, $u_{n}=0$. The proof of Theorem 4.1 is complete in the case $0<|\tilde{n}| \leq \delta_{0}^{\frac{3}{2}} \nu^{-\frac{3}{4}}$.

\section{Analysis in high frequency $|\tilde{n}| \geq \delta \nu^{-\frac{3}{4}}$}

In this section we consider the linearized problem in the regime $|\tilde{n}| \geq \delta \nu^{-\frac{3}{4}}$. If $|\tilde{n}| \gg \nu^{-\frac{3}{4}}$ then the problem is easy since the dissipation is strong enough. Hence we must at least handle the regime $|\tilde{n}| \sim O\left(\nu^{-\frac{3}{4}}\right)$, in which the strength of the boundary layer is the same order as the dissipation, and this fact leads to an essential difficulty in constructing the boundary layer corrector, in particular the lower bound of the fast mode, which was the key in the previous section.

To overcome this difficulty we go back to the energy argument for the velocity in the original variables and try to gain the coercive estimate from the convection $U_{s}^{\nu} \partial_{x}$. This will be achieved from the imaginary part of the energy identity, rather than the positive part of it. To be precise let us recall the problem

$$
\left\{\begin{array}{l}
i \tilde{n} U_{s}^{\nu} u_{n}+u_{n, 2}\left(\partial_{y} U_{s}^{\nu}\right) \mathbf{e}_{1}-\nu\left(\partial_{y}^{2}-\tilde{n}^{2}\right) u_{n}+\left(\begin{array}{c}
i \tilde{n} p_{n} \\
\partial_{y} p_{n}
\end{array}\right)=f_{n}, \quad y>0 \\
i \tilde{n} u_{n, 1}+\partial_{y} u_{n, 2}=0, \quad y>0 \\
\left.u_{n}\right|_{y=0}=0
\end{array}\right.
$$

Here $u_{n}=\left(\partial_{y} \phi_{n},-i \tilde{n} \phi_{n}\right)^{\top}$ with the streamfunction $\phi_{n}=\phi_{n}(y)$. The following proposition is valid for the regime $|\tilde{n}| \nu^{\frac{1}{2}} \gg 1$. 
Proposition 8.1. There exists a positive number $\delta_{2}>0$ such that if $|\tilde{n}| \geq \delta_{2}^{-1} \nu^{-\frac{1}{2}}$ then there exists a unique solution $u_{n} \in H^{2}\left(\mathbb{R}_{+}\right)^{2} \cap H_{0}^{1}\left(\mathbb{R}_{+}\right)^{2}$ to (8.1) satisfying the following estimates: (i) if $|\tilde{n}| \geq \delta_{2}^{-1} \nu^{-\frac{3}{4}}$ then

$$
\begin{aligned}
\left\|u_{n}\right\|_{L^{2}} & \leq \frac{C}{|\tilde{n}|^{2} \nu}\left\|f_{n}\right\|_{L^{2}}, \\
\left\|\partial_{y} u_{n}\right\|_{L^{2}}+|\tilde{n}|\left\|u_{n}\right\|_{L^{2}} & \leq \frac{C}{|\tilde{n}| \nu}\left\|f_{n}\right\|_{L^{2}} .
\end{aligned}
$$

(ii) if $\delta_{2}^{-1} \nu^{-\frac{1}{2}} \leq|\tilde{n}| \leq \delta_{2}^{-1} \nu^{-\frac{3}{4}}$ then

$$
\begin{aligned}
\left\|u_{n}\right\|_{L^{2}} & \leq \frac{C}{|\tilde{n}|^{\frac{4}{5}} \nu^{\frac{1}{10}}}\left\|f_{n}\right\|_{L^{2}}, \\
\left\|\partial_{y} u_{n}\right\|_{L^{2}}+|\tilde{n}|\left\|u_{n}\right\|_{L^{2}} & \leq \frac{C}{|\tilde{n}|^{\frac{3}{5}} \nu^{\frac{7}{10}}}\left\|f_{n}\right\|_{L^{2}} .
\end{aligned}
$$

Remark 8.2. When $|\tilde{n}| \sim \nu^{-\frac{3}{4}}$ we have $|\tilde{n}|^{-\frac{4}{5}} \nu^{-\frac{1}{10}} \sim|\tilde{n}|^{-\frac{2}{3}}$ and $|\tilde{n}|^{-\frac{3}{5}} \nu^{-\frac{7}{10}} \sim|\tilde{n}|^{-\frac{1}{3}} \nu^{-\frac{1}{2}}$.

Proof. We may assume that $\tilde{n}>0$. We focus on the a priori estimate. By taking the inner product with $u_{n}$ in the first equation of (8.1), we have

$$
i \tilde{n}\left\langle U_{s}^{\nu} u_{n}, u_{n}\right\rangle+\left\langle u_{n, 2} \partial_{y} U_{s}^{\nu}, u_{n, 1}\right\rangle+\nu\left(\left\|\partial_{y} u_{n}\right\|_{L^{2}}^{2}+\tilde{n}^{2}\left\|u_{n}\right\|_{L^{2}}^{2}\right)=\left\langle f_{n}, u_{n}\right\rangle .
$$

The real part of this identity gives

$$
\nu\left(\left\|\partial_{y} u_{n}\right\|_{L^{2}}^{2}+\tilde{n}^{2}\left\|u_{n}\right\|_{L^{2}}^{2}\right)=-\operatorname{Re}\left\langle u_{n, 2} \partial_{y} U_{s}^{\nu}, u_{n, 1}\right\rangle+\operatorname{Re}\left\langle f_{n}, u_{n}\right\rangle,
$$

while the imaginary part of this identity gives

$$
\tilde{n}\left(\left\langle U_{s}^{\nu} u_{n}, u_{n}\right\rangle-\operatorname{Re}\left\langle\left(\partial_{y} U_{s}^{\nu}\right) \phi_{n}, \partial_{y} \phi_{n}\right\rangle\right)=\mathcal{I} m\left\langle f_{n}, u_{n}\right\rangle .
$$

Below we take $\delta_{2} \in(0,1)$ small enough depending only on $U_{s}$.

(i) Case $\tilde{n} \geq \delta_{2}^{-1} \nu^{-\frac{3}{4}}$ : The first term in the right-hand side of (8.7) is estimated as

$$
\begin{aligned}
\left|-\operatorname{Re}\left\langle u_{n, 2} \partial_{y} U_{s}^{\nu}, u_{n, 1}\right\rangle\right| \leq \nu^{-\frac{1}{2}}\left\|\partial_{Y} U_{s}\right\|_{L^{\infty}}\left\|u_{n}\right\|_{L^{2}}^{2} & =\nu \tilde{n}^{2} \frac{\left\|\partial_{Y} U_{s}\right\|_{L^{\infty}}}{\nu^{\frac{3}{2}} \tilde{n}^{2}}\left\|u_{n}\right\|_{L^{2}}^{2} \\
& \leq \frac{\nu \tilde{n}^{2}}{2}\left\|u_{n}\right\|_{L^{2}}^{2}
\end{aligned}
$$

if $\delta_{2}>0$ is small enough. Thus we have

$$
\nu\left(\left\|\partial_{y} u_{n}\right\|_{L^{2}}^{2}+\tilde{n}^{2}\left\|u_{n}\right\|_{L^{2}}^{2}\right) \leq 2\left\|f_{n}\right\|_{L^{2}}\left\|u_{n}\right\|_{L^{2}},
$$

which proves (8.2)-(8.3).

(ii) Case $\delta_{2}^{-1} \nu^{-\frac{1}{2}} \leq \tilde{n} \leq \delta_{2}^{-1} \nu^{-\frac{3}{4}}$ : We use the imaginary part of the energy identity (8.8), and then from the integration by parts,

$$
\int_{0}^{\infty} U_{s}^{\nu}\left(\left|\partial_{y} \phi_{n}\right|^{2}+\tilde{n}^{2}\left|\phi_{n}\right|^{2}\right) d y+\frac{1}{2} \int_{0}^{\infty}\left(\partial_{y}^{2} U_{s}^{\nu}\right)\left|\phi_{n}\right|^{2} d y=\frac{1}{\tilde{n}} \mathcal{I} m\left\langle f_{n}, u_{n}\right\rangle
$$


To estimate the second term in the left-hand side of this equality, we use the condition $\left|\partial_{y}^{2} U_{s}^{\nu}\right| \leq C \nu^{-\frac{1}{2}} \partial_{y} U_{s}^{\nu}$ for $0 \leq y \leq 2 Y_{0} \nu^{\frac{1}{2}}$, see Proposition 2.1. Let $\chi(Y)$ be a cut-off such that $\chi(Y)=1$ for $0 \leq Y \leq Y_{0}$ and $\chi(Y)=0$ for $Y \geq 2 Y_{0}$. Then we have

$$
\begin{aligned}
\left.\left|\frac{1}{2} \int_{0}^{\infty}\left(\partial_{y}^{2} U_{s}^{\nu}\right)\right| \phi_{n}\right|^{2} d y \mid & \leq \frac{C}{2} \nu^{-\frac{1}{2}} \int_{0}^{\infty} \partial_{y} U_{s}^{\nu} \chi\left(\frac{y}{\sqrt{\nu}}\right)\left|\phi_{n}\right|^{2} d y+C \nu^{-1} \int_{Y_{0} \nu^{\frac{1}{2}}}^{\infty}\left|\phi_{n}\right|^{2} d y \\
& =-\frac{C}{2} \nu^{-\frac{1}{2}} \int_{0}^{\infty} U_{s}^{\nu} \partial_{y}\left(\chi\left(\frac{y}{\sqrt{\nu}}\right)\left|\phi_{n}\right|^{2}\right) d y+C \nu^{-1} \int_{Y_{0} \nu^{\frac{1}{2}}}^{\infty}\left|\phi_{n}\right|^{2} d y \\
& \leq C \nu^{-1} \int_{Y_{0} \nu^{\frac{1}{2}}}^{\infty} U_{s}^{\nu}\left|\phi_{n}\right|^{2} d y-C \nu^{-\frac{1}{2}} \operatorname{Re} \int_{0}^{\infty} U_{s}^{\nu} \chi\left(\frac{y}{\sqrt{\nu}}\right) \partial_{y} \phi_{n} \overline{\phi_{n}} d y \\
& \leq \frac{C}{\nu \tilde{n}^{2}}\left\|\sqrt{U_{s}^{\nu}} \tilde{n} \phi_{n}\right\|_{L^{2}}^{2}+\frac{C}{\nu^{\frac{1}{2}} \tilde{n}}\left\|\sqrt{U_{s}^{\nu}} \partial_{y} \phi_{n}\right\|_{L^{2}}\left\|\sqrt{U_{s}^{\nu}} \tilde{n} \phi_{n}\right\|_{L^{2}} .
\end{aligned}
$$

Here we have used $\left|\frac{1}{U_{s}^{\nu}(y)}\right| \leq C$ for $y \geq Y_{0} \nu^{\frac{1}{2}}$. Hence if $\nu^{\frac{1}{2}} \tilde{n}$ is large enough, the term $\frac{1}{2} \int_{0}^{\infty}\left(\partial_{y}^{2} U_{s}^{\nu}\right)\left|\phi_{n}\right|^{2} d y$ is absorbed by the first term in the left-hand side, resulting in

$$
\left\|\sqrt{U_{s}^{\nu}} u_{n}\right\|_{L^{2}}^{2} \leq \frac{2}{\tilde{n}} \mathcal{I} m\left\langle f_{n}, u_{n}\right\rangle .
$$

The estimate (8.9) is coercive but degenerate near the boundary $y=0$. To recover the estimate near the boundary we use the real part of the energy identity (8.7). To this end we recall the interpolation inequality (2.1), which is formulated in the rescaled variable $Y$. In the original variable, it can be written as

$$
\|g\|_{L^{2}}^{2} \leq C \nu^{\frac{1}{3}}\left\|\sqrt{U_{s}^{\nu}} g\right\|_{L^{2}}^{\frac{4}{3}}\left\|\partial_{y} g\right\|_{L^{2}}^{\frac{2}{3}}+C\left\|\sqrt{U_{s}^{\nu}} g\right\|_{L^{2}}^{2}, \quad g=g(y) \in H_{0}^{1}\left(\mathbb{R}_{+}\right) .
$$

This yields from (8.7) that

$$
\left\|u_{n}\right\|_{L^{2}}^{2} \leq C \nu^{\frac{1}{3}}\left\|\sqrt{U_{s}^{\nu}} u_{n}\right\|_{L^{2}}^{\frac{4}{3}}\left(-\nu^{-1} \operatorname{Re}\left\langle u_{n, 2} \partial_{y} U_{s}^{\nu}, u_{n, 1}\right\rangle+\nu^{-1} \operatorname{Re}\left\langle f_{n}, u_{n}\right\rangle\right)^{\frac{1}{3}}+C\left\|\sqrt{U_{s}^{\nu}} u_{n}\right\|_{L^{2}}^{2} .
$$

By using the Hardy inequality and the divergence free condition we have

$$
\begin{aligned}
\left|\operatorname{Re}\left\langle u_{n, 2} \partial_{y} U_{s}^{\nu}, u_{n, 1}\right\rangle\right| & \leq\left\|\frac{y^{\frac{1}{2}} \partial_{y} U_{s}^{\nu}}{\sqrt{U_{s}^{\nu}}}\right\|_{L^{\infty}}\left\|\frac{u_{n, 2}}{y^{\frac{1}{2}}}\right\|_{L^{2}}\left\|\sqrt{U_{s}^{\nu}} u_{n, 1}\right\|_{L^{2}} \\
& \leq C \nu^{-\frac{1}{4}}\left\|\frac{Y^{\frac{1}{2}} \partial_{Y} U_{s}}{\sqrt{U_{s}}}\right\|_{L_{Y}^{\infty}}\left\|u_{n, 2}\right\|_{L^{2}}^{\frac{1}{2}}\left\|\partial_{y} u_{n, 2}\right\|_{L^{2}}^{\frac{1}{2}}\left\|\sqrt{U_{s}^{\nu}} u_{n, 1}\right\|_{L^{2}} \\
& \leq \frac{C \tilde{n}^{\frac{1}{2}}}{\nu^{\frac{1}{4}}}\left\|u_{n}\right\|_{L^{2}}\left\|\sqrt{U_{s}^{\nu}} u_{n}\right\|_{L^{2}} .
\end{aligned}
$$

Therefore,

$$
\begin{aligned}
\left\|u_{n}\right\|_{L^{2}}^{2} & \leq C \nu^{\frac{1}{3}}\left\|\sqrt{U_{s}^{\nu}} u_{n}\right\|_{L^{2}}^{\frac{4}{3}}\left(\nu^{-\frac{5}{4}} \tilde{n}^{\frac{1}{2}}\left\|\sqrt{U_{s}^{\nu}} u_{n}\right\|_{L^{2}}\left\|u_{n}\right\|_{L^{2}}+\nu^{-1}\left|\operatorname{Re}\left\langle f_{n}, u_{n}\right\rangle\right|\right)^{\frac{1}{3}}+C\left\|\sqrt{U_{s}^{\nu}} u_{n}\right\|_{L^{2}}^{2} \\
& \leq C \nu^{-\frac{1}{12}} \tilde{n}^{\frac{1}{6}}\left\|\sqrt{U_{s}^{\nu}} u_{n}\right\|_{L^{2}}^{\frac{5}{3}}\left\|u_{n}\right\|_{L^{2}}^{\frac{1}{3}}+C\left\|\sqrt{U_{s}^{\nu}} u_{n}\right\|_{L^{2}}^{\frac{4}{3}}\left|\operatorname{Re}\left\langle f_{n}, u_{n}\right\rangle\right|^{\frac{1}{3}}+C\left\|\sqrt{U_{s}^{\nu}} u_{n}\right\|_{L^{2}}^{2},
\end{aligned}
$$

which gives from (8.9) and $\tilde{n} \geq \nu^{-\frac{1}{2}}$,

$$
\begin{aligned}
\left\|u_{n}\right\|_{L^{2}}^{2} & \leq C\left(\nu^{-\frac{1}{10}} \tilde{n}^{\frac{1}{5}}+1\right)\left\|\sqrt{U_{s}^{\nu}} u_{n}\right\|_{L^{2}}^{2}+C\left\|\sqrt{U_{s}^{\nu}} u_{n}\right\|_{L^{2}}^{\frac{4}{3}}\left|\operatorname{Re}\left\langle f_{n}, u_{n}\right\rangle\right|^{\frac{1}{3}} \\
& \leq C \nu^{-\frac{1}{10}} \tilde{n}^{\frac{1}{5}-1}\left|\mathcal{I} m\left\langle f_{n}, u_{n}\right\rangle\right|+C \tilde{n}^{-\frac{2}{3}}\left|\mathcal{I} m\left\langle f_{n}, u_{n}\right\rangle\right|^{\frac{2}{3}}\left|\operatorname{Re}\left\langle f_{n}, u_{n}\right\rangle\right|^{\frac{1}{3}} \\
& \leq C\left(\nu^{-\frac{1}{10}} \tilde{n}^{-\frac{4}{5}}+\tilde{n}^{-\frac{2}{3}}\right)\left\|f_{n}\right\|_{L^{2}}\left\|u_{n}\right\|_{L^{2}},
\end{aligned}
$$


that is,

$$
\left\|u_{n}\right\|_{L^{2}} \leq C\left(\nu^{-\frac{1}{10}} \tilde{n}^{-\frac{4}{5}}+\tilde{n}^{-\frac{2}{3}}\right)\left\|f_{n}\right\|_{L^{2}} \leq C \nu^{-\frac{1}{10}} \tilde{n}^{-\frac{4}{5}}\left\|f_{n}\right\|_{L^{2}}
$$

Here we have used the condition $\tilde{n} \leq \delta_{2}^{-1} \nu^{-\frac{3}{4}}$. Then (8.11) and (8.9) imply

$$
\left|\operatorname{Re}\left\langle u_{n, 2} \partial_{y} U_{s}^{\nu}, u_{n, 1}\right\rangle\right| \leq C \nu^{-\frac{1}{4}}\left\|f_{n}\right\|_{L^{2}}^{\frac{1}{2}}\left\|u_{n}\right\|_{L^{2}}^{\frac{3}{2}} .
$$

Thus the estimate for the derivatives is obtained from (8.7):

$$
\begin{aligned}
\left\|\partial_{y} u_{n}\right\|_{L^{2}}^{2}+\tilde{n}^{2}\left\|u_{n}\right\|_{L^{2}}^{2} & \leq C \nu^{-\frac{5}{4}}\left\|f_{n}\right\|_{L^{2}}^{\frac{1}{2}}\left\|u_{n}\right\|_{L^{2}}^{\frac{3}{2}}+\nu^{-1}\left\|f_{n}\right\|_{L^{2}}\left\|u_{n}\right\|_{L^{2}} \\
& \leq C\left(\tilde{n}^{-\frac{6}{5}} \nu^{-\frac{7}{5}}+\tilde{n}^{-\frac{4}{5}} \nu^{-\frac{11}{10}}\right)\left\|f_{n}\right\|_{L^{2}}^{2} \\
& \leq C \tilde{n}^{-\frac{6}{5}} \nu^{-\frac{7}{5}}\left\|f_{n}\right\|_{L^{2}}^{2} .
\end{aligned}
$$

The proof of the a priori estimates is complete, which also implies the uniqueness. As for the existence, we first replace the operator $-\nu\left(\partial_{y}^{2}-\tilde{n}^{2}\right)$ by $-\nu\left(\partial_{y}^{2}-\tilde{n}^{2}\right)+l$ with $l>0$, and if $l$ is large enough then it is easy to show the unique existence of the solution for any $f_{n} \in L^{2}\left(\mathbb{R}_{+}\right)^{2}$. One can check that the argument above for the a priori estimates is valid even for $l>0$ without any changes, and all of the a priori estimates are uniform in $l>0$. Thus, the existence of the solution for the case $l=0$ holds by the continuity. The proof is complete.

Proposition 8.1 shows Theorem 4.1 for $\tilde{n} \geq \delta_{0}^{\frac{3}{2}} \nu^{-\frac{3}{4}}$ as long as $\delta_{2}^{-1} \nu^{-\frac{1}{2}} \leq \delta_{0}^{\frac{3}{2}} \nu^{-\frac{3}{4}}$, which is valid for sufficiently small $\nu>0$. The proof of Theorem 4.1 is complete.

\section{Nonlinear problem}

In this section we prove Theorem 1.1 based on the linear result in the previous section, Theorems 3.1 and 4.1. The proof relies on the fixed point theorem for the contraction map. Let us denote by $X_{\nu, \epsilon}$ the closed convex set defined by

$$
\begin{aligned}
& X_{\nu, \epsilon}=\{u \in X \mid \\
& \left.\|u\|_{X_{\nu}}:=\left\|u_{0,1}\right\|_{L^{\infty}}+\nu^{\frac{1}{4}}\left\|\partial_{y} u_{0,1}\right\|_{L^{2}}+\sum_{n \neq 0}\left\|u_{n}\right\|_{L^{\infty}}+\nu^{-\frac{1}{4}}\left\|\mathcal{Q}_{0} u\right\|_{L^{2}}+\nu^{\frac{1}{4}}\left\|\nabla \mathcal{Q}_{0} u\right\|_{L^{2}} \leq \epsilon \frac{\nu^{\frac{1}{2}}}{|\log \nu|^{\frac{1}{2}}}\right\} .
\end{aligned}
$$

Then for $w \in X_{\nu, \epsilon}$ we define the map $\Psi[w]=u$ as the solution to the linear problem

$$
\left\{\begin{array}{l}
U_{s}^{\nu} \partial_{x} u+u_{2} \partial_{y} U_{s}^{\nu} \mathbf{e}_{1}-\nu \Delta u+\nabla p=-w \cdot \nabla w+f^{\nu}, \quad(x, y) \in \mathbb{T}_{\kappa} \times \mathbb{R}_{+}, \\
\operatorname{div} u^{\nu}=0, \quad(x, y) \in \mathbb{T}_{\kappa} \times \mathbb{R}_{+}, \\
\left.u^{\nu}\right|_{y=0}=0
\end{array}\right.
$$

We observe that

$$
-w \cdot \nabla w=-w_{0,1} \partial_{x} \mathcal{Q}_{0} w-\mathcal{Q}_{0} w_{2} \partial_{y} w_{0,1} \mathbf{e}_{1}-\mathcal{Q}_{0} w \cdot \nabla \mathcal{Q}_{0} w
$$

and therefore,

$$
-\mathcal{P}_{0}(w \cdot \nabla w)=-\mathcal{P}_{0}\left(\mathcal{Q}_{0} w \cdot \nabla \mathcal{Q}_{0} w\right)=-\partial_{y} \mathcal{P}_{0}\left(\mathcal{Q}_{0} w_{2} \mathcal{Q}_{0} w\right)
$$


Hence the zero mode of the source term in (9.2) is written as

$$
\mathcal{P}_{0}\left(-w \cdot \nabla w+f^{\nu}\right)=\partial_{y}\left(-\mathcal{P}_{0}\left(\mathcal{Q}_{0} w_{2} \mathcal{Q}_{0} w\right)\right)=: \partial_{y} H[w] .
$$

Thus, Theorem 3.1 shows

$$
\begin{aligned}
& \left\|u_{0,1}\right\|_{L^{\infty}} \leq \frac{1}{\nu}\left\|H[w]_{1}\right\|_{L^{1}} \leq \frac{1}{\nu}\left\|\mathcal{Q}_{0} w\right\|_{L^{2}}^{2}, \\
& \left\|\partial_{y} u_{0,1}\right\|_{L^{2}} \leq \frac{1}{\nu}\left\|H[w]_{1}\right\|_{L^{2}} \leq \frac{1}{\nu}\left\|\mathcal{Q}_{0} w\right\|_{L^{\infty}}\left\|\mathcal{Q}_{0} w\right\|_{L^{2}}, \\
& \left.u_{0,1}\right|_{y=0}=0 .
\end{aligned}
$$

As for the nonzero mode, we see

$$
\mathcal{Q}_{0}\left(-w \cdot \nabla w+f^{\nu}\right)=-w_{0,1} \partial_{x} \mathcal{Q}_{0} w-\mathcal{Q}_{0} w_{2} \partial_{y} w_{0,1} \mathbf{e}_{1}-\mathcal{Q}_{0}\left(\mathcal{Q}_{0} w \cdot \nabla \mathcal{Q}_{0} w\right)+f^{\nu} .
$$

Therefore, Theorem 4.1 implies, by applying the Parseval equality,

$$
\begin{aligned}
& \left\|\mathcal{Q}_{0} u\right\|_{L^{2}} \\
& \leq C\left\|-w_{0,1} \partial_{x} \mathcal{Q}_{0} w-\mathcal{Q}_{0} w_{2} \partial_{y} w_{0,1} \mathbf{e}_{1}-\mathcal{Q}_{0}\left(\mathcal{Q}_{0} w \cdot \nabla \mathcal{Q}_{0} w\right)+f^{\nu}\right\|_{L^{2}} \\
& \leq C\left(\left\|w_{0,1}\right\|_{L^{\infty}}\left\|\nabla \mathcal{Q}_{0} w\right\|_{L^{2}}+\left\|\mathcal{Q}_{0} w\right\|_{L^{\infty}}\left\|\partial_{y} w_{0,1}\right\|_{L^{2}}+\left\|\mathcal{Q}_{0} w\right\|_{L^{\infty}}\left\|\nabla \mathcal{Q}_{0} w\right\|_{L^{2}}+\left\|f^{\nu}\right\|_{L^{2}}\right),
\end{aligned}
$$

and similarly,

$$
\begin{aligned}
& \left\|\nabla \mathcal{Q}_{0} u\right\|_{L^{2}} \\
& \leq \frac{C}{\nu^{\frac{1}{2}}}\left(\left\|w_{0,1}\right\|_{L^{\infty}}\left\|\nabla \mathcal{Q}_{0} w\right\|_{L^{2}}+\left\|\mathcal{Q}_{0} w\right\|_{L^{\infty}}\left\|\partial_{y} w_{0,1}\right\|_{L^{2}}+\left\|\mathcal{Q}_{0} w\right\|_{L^{\infty}}\left\|\nabla \mathcal{Q}_{0} w\right\|_{L^{2}}+\left\|f^{\nu}\right\|_{L^{2}}\right) .
\end{aligned}
$$

For $n \neq 0$, by using the identity

$$
\mathcal{P}_{n}\left(-w \cdot \nabla w+f^{\nu}\right)=-w_{0,1} \partial_{x} \mathcal{P}_{n} w-\mathcal{P}_{n} w_{2} \partial_{y} w_{0,1} \mathbf{e}_{1}-\mathcal{P}_{n}\left(\mathcal{Q}_{0} w \cdot \nabla \mathcal{Q}_{0} w\right)+\mathcal{P}_{n} f^{\nu},
$$

we have from Theorem 4.1,

$\left\|\mathcal{P}_{n} u\right\|_{L^{\infty}}$

$$
\leq\left\{\begin{array}{l}
\frac{C}{|\tilde{n}|^{\frac{1}{2}} \nu^{\frac{1}{4}}}\left(\left\|w_{0,1}\right\|_{L^{\infty}}\left\|\nabla \mathcal{P}_{n} w\right\|_{L^{2}}+\left\|\mathcal{P}_{n} w\right\|_{L^{\infty}}\left\|\partial_{y} w_{0,1}\right\|_{L^{2}}+\left\|\mathcal{P}_{n}\left(\mathcal{Q}_{0} w \cdot \nabla \mathcal{Q}_{0} w\right)\right\|_{L^{2}}+\left\|\mathcal{P}_{n} f^{\nu}\right\|_{L^{2}}\right), \\
\text { if } \quad 0<|\tilde{n}| \leq \delta_{*} \nu^{-\frac{3}{4}}, \\
\frac{C}{|\tilde{n}|^{\frac{3}{2}} \nu}\left(\left\|w_{0,1}\right\|_{L^{\infty}}\left\|\nabla \mathcal{P}_{n} w\right\|_{L^{2}}+\left\|\mathcal{P}_{n} w\right\|_{L^{\infty}}\left\|\partial_{y} w_{0,1}\right\|_{L^{2}}+\left\|\mathcal{P}_{n}\left(\mathcal{Q}_{0} w \cdot \nabla \mathcal{Q}_{0} w\right)\right\|_{L^{2}}+\left\|\mathcal{P}_{n} f^{\nu}\right\|_{L^{2}}\right), \\
\text { if }|\tilde{n}|>\delta_{*} \nu^{-\frac{3}{4}}
\end{array}\right.
$$

Note that the $L^{\infty}$ estimate in the case $|\tilde{n}| \geq \nu^{-\frac{1}{2}}$ follows from (ii) and (iii) in Theorem 4.1 combined with the interpolation $\left\|\mathcal{P}_{n} u\right\|_{L^{\infty}} \leq C\left\|\partial_{y} u_{n}\right\|_{L_{y}^{2}}^{\frac{1}{2}}\left\|u_{n}\right\|_{L_{y}^{2}}^{\frac{1}{2}}$. Thus, by the Parseval equality,

$$
\begin{gathered}
\sum_{n \neq 0}\left\|\mathcal{P}_{n} u\right\|_{L^{\infty}} \leq \frac{C}{\nu^{\frac{1}{4}}}\left(|\log \nu|^{\frac{1}{2}}\left\|w_{0,1}\right\|_{L^{\infty}}\left\|\nabla \mathcal{Q}_{0} w\right\|_{L^{2}}+\sum_{n \neq 0}\left\|\mathcal{P}_{n} w\right\|_{L^{\infty}}\left\|\partial_{y} w_{0,1}\right\|_{L^{2}}\right. \\
\left.\quad+|\log \nu|^{\frac{1}{2}}\left\|\mathcal{Q}_{0}\left(\mathcal{Q}_{0} w \cdot \nabla \mathcal{Q}_{0} w\right)\right\|_{L^{2}}+|\log \nu|^{\frac{1}{2}}\left\|\mathcal{Q}_{0} f^{\nu}\right\|_{L^{2}}\right) \\
\leq \frac{C}{\nu^{\frac{1}{4}}}\left(|\log \nu|^{\frac{1}{2}}\left\|w_{0,1}\right\|_{L^{\infty}}\left\|\nabla \mathcal{Q}_{0} w\right\|_{L^{2}}+\sum_{n \neq 0}\left\|\mathcal{P}_{n} w\right\|_{L^{\infty}}\left\|\partial_{y} w_{0,1}\right\|_{L^{2}}\right. \\
\left.\quad+|\log \nu|^{\frac{1}{2}}\left\|\mathcal{Q}_{0} w\right\|_{L^{\infty}}\left\|\nabla \mathcal{Q}_{0} w\right\|_{L^{2}}+|\log \nu|^{\frac{1}{2}}\left\|f^{\nu}\right\|_{L^{2}}\right) .
\end{gathered}
$$


We note that $\left\|\mathcal{Q}_{0} w\right\|_{L^{\infty}} \leq C \sum_{n \neq 0}\left\|\mathcal{P}_{n} w\right\|_{L^{\infty}}$ holds. Collecting these, we have

$$
\|\Psi[w]\|_{X_{\nu}} \leq \frac{C|\log \nu|^{\frac{1}{2}}}{\nu^{\frac{1}{2}}}\|w\|_{X_{\nu}}^{2}+\frac{C|\log \nu|^{\frac{1}{2}}}{\nu^{\frac{1}{4}}}\left\|f^{\nu}\right\|_{L^{2}}
$$

and since the nonlinear term $-w \cdot \nabla w$ is quadratic, we can show a similar bound for the difference $\Psi[w]-\Psi\left[w^{\prime}\right]$ :

$$
\left\|\Psi[w]-\Psi\left[w^{\prime}\right]\right\|_{X_{\nu}} \leq \frac{C|\log \nu|^{\frac{1}{2}}}{\nu^{\frac{1}{2}}}\left(\|w\|_{X_{\nu}}+\left\|w^{\prime}\right\|_{X_{\nu}}\right)\left\|w-w^{\prime}\right\|_{X_{\nu}} .
$$

Hence $\Psi$ is a contraction map from $X_{\nu, \epsilon}$ into itself if $\epsilon$ is small enough and $\left\|f^{\nu}\right\|_{L^{2}}$ is small enough compared with $\epsilon|\log \nu|^{-1} \nu^{\frac{1}{4}+\frac{1}{2}}$. By the standard fixed point theorem there exists a unique fixed point $u^{*}$ of $\Psi$ in $X_{\nu, \epsilon}$, which is the unique solution to (1.7) in $X_{\nu, \epsilon}$. Note that the solution $u^{*}$ satisfies $\left\|u^{*}\right\|_{X_{\nu}} \leq C|\log \nu|^{\frac{1}{2}} \nu^{-\frac{1}{4}}\left\|f^{\nu}\right\|_{L^{2}}$, which gives (1.11). Since

$$
g=-U_{s}^{\nu} \partial_{x} u^{*}-u_{2}^{*} \partial_{y} U_{s}^{\nu} \mathbf{e}_{1}-u^{*} \cdot \nabla u^{*}+f^{\nu}
$$

belongs to $L^{2}\left(\mathbb{T}_{\kappa} \times \mathbb{R}_{+}\right)^{2}$, the elliptic regularity of the Stokes equations also implies $\nabla^{2} u^{*} \in$ $L^{2}\left(\mathbb{T}_{\kappa} \times \mathbb{R}_{+}\right)$and $\nabla p^{*} \in L^{2}\left(\mathbb{T}_{\kappa} \times \mathbb{R}_{+}\right)^{2}$. The proof is complete.

\section{References}

[1] Abramowitz, M. and Stegun, I.; Handbook of mathematical functions with formulas, graphs, and mathematical tables, National Bureau of Standards Applied Mathematics Series, 55,1964, 1046 pp.

[2] Alexandre, R., Wang, Y.-G., Xu, C.-J., and Yang, T.; Well-posedness of the Prandtl equation in Sobolev spaces. J. Amer. Math. Soc., 28(3):745-784, 2015.

[3] Cowley, S.; Laminar boundary layer theory: a 20th century paradox ? Mechanics for a new millenium, 389-412, 2001 Kluwer Academic Publisher.

[4] Dalibard, A.-L., and Masmoudi, N.; Phénomène de séparation pour l'équation de Prandtl. Séminaire Laurent Schwartz, Exp IX, 18. Ed. Ecole Polytechnique, 2016.

[5] Drazin, P. G., and Reid,W. H.; Hydrodynamic stability. Cambridge Mathematical Library. Cambridge University Press, Cambridge, second edition, 2004. With a foreword by John Miles.

[6] E, W.; Boundary layer theory and the zero-viscosity limit of the Navier-Stokes equation. Acta Math. Sin. 16, 2 (2000) 207-218.

[7] Fei, M., Tao, T., and Zhang, Z.; On the zero-viscosity limit of the Navier-Stokes equations in $\mathbb{R}_{+}^{3}$ without analyticity. J. Math. Pures Appl., 112:170-229, 2018.

[8] Gallagher, I., Higaki, M., and Maekawa, Y.; On stationary two-dimensional flows around a fast rotating disk. arXiv:1710.01029.

[9] Gargano, F., Sammartino, M., and Sciacca, V.; Singularity formation for Prandtl's equations. Phys. D, 238(19):1975-1991, 2009.

[10] Gerard-Varet, D., and Dormy, E.; On the ill-posedness of the Prandtl equation. J. Amer. Math. Soc., 23(2):591-609, 2010.

[11] Gerard-Varet, D., Maekawa, Y., and Masmoudi, N.; Gevrey stability of Prandtl expansions for 2D Navier-Stokes flows. Accepted by Duke Math. J. (available in ArXiv e-prints, July 2016)

[12] Gerard-Varet, D., and Masmoudi, N.; Well-posedness for the Prandtl system without analyticity or monotonicity. Ann. Scient. Ec. Norm. Sup., 48(4):1273-1325, 2015.

[13] Gerard-Varet, D., and Prestipino, M.; Formal derivation and stability analysis of boundary layer models in MHD. Z. Angew. Math. Phys. 68 (3), Art. 76 (2017). 
[14] Goldstein, S.; On laminar boundary layer flow near a position of separation. Quaterly J. Mech. Applied Math. 1, 43-69, 1948.

[15] Grenier, E.; On the nonlinear instability of Euler and Prandtl equations. Comm. Pure Appl. Math., 53(9):1067-1091, 2000.

[16] Grenier, E., Guo, Y., and Nguyen, T.; Spectral instability of characteristic boundary layer flows. Duke Math. J., 165:3085-3146, 2016.

[17] Grenier, E., and Nguyen, T.; Sharp bounds on linear semigroup of Navier-Stokes with boundary layer norms. Preprint arXiv, 2017.

[18] Guo, Y., Iyer, S.; Validity of steady Prandtl layer expansions. arXiv:1805.05891, May 2018.

[19] Guo, Y., Nguyen, T.; Prandtl boundary layer expansions of steady Navier-Stokes flows over a moving plate. Ann. PDE 3 (2017), no. 1, Art. 10, 58 pp.

[20] Hong, L., and Hunter, J.; Singularity formation and instability in the unsteady inviscid and viscous Prandtl equations. Comm. Math. Sci., 1:293-316, 2003.

[21] Iyer, S.; Steady Prandtl boundary layer expansions over a rotating disk. Arch. Ration. Mech. Anal. 224 (2017), no. 2, 421-469.

[22] Kukavica, I., and Vicol, V.; On the local existence of analytic solutions to the Prandtl boundary layer equations. Commun. Math. Sci., 11(1):269-292, 2013.

[23] Li, W.-X., and Yang., T.; Well-posedness in Gevrey space for the Prandtl equations with nondegenerate critical points To appear in J. Eur. Math. Soc.

[24] Liu, C., Xie, F., and Yang, T.; MHD boundary layers in Sobolev spaces without monotonicity. I. Well-posedness theory, to appear in CPAM.

[25] Liu, C., Xie, F., and Yang, T.; MHD boundary layers in Sobolev spaces without monotonicity. II. Convergence theory, preprint arXiv 2017.

[26] Lombardo, M. C., Cannone, M., and Sammartino, M.; Well-posedness of the boundary layer equations. SIAM J. Math. Anal., 35(4):987-1004, 2003.

[27] Lopes Filho, M. C., Mazzucato, A. L., and Nussenzveig Lopes H. J.; Vanishing viscosity limit for incompressible flow inside a rotating circle. Phys. D, 237(10-12):1324-1333, 2008.

[28] Maekawa, Y.; On the inviscid limit problem of the vorticity equations for viscous incompressible flows in the half plane, Comm. Pure and Applied Math., 67:1045-1128, 2014.

[29] Masmoudi, N., and Wong, T. K.; Local-in-time existence and uniqueness of solutions to the Prandtl equations by energy methods. Comm. Pure Appl. Math., 68(10):1683-1741, 2015.

[30] Matsui, S., and Shirota, T.; On separation points of solutions to Prandtl boundary layer problem. Hokkaido Math. J., 13(1): 92-108, 1984.

[31] Mazzucato, A. L., and Taylor, M. E.; Vanishing viscosity plane parallel channel flow and related singular perturbation problems. Analysis \& PDE, 1(1):35-93, 2008.

[32] Oleinik, O. A., and Samokhin.,V. N.; Mathematical models in boundary layer theory, volume 15 of Applied Mathematics and Mathematical Computation. Chapman \& Hall/CRC, Boca Raton, FL, 1999.

[33] Sammartino, M., and Caflisch, R. E.; Zero viscosity limit for analytic solutions, of the NavierStokes equation on a half-space. I. Existence for Euler and Prandtl equations. Comm. Math. Phys., 192(2):433-461, 1998.

[34] Sammartino, M., and Caflisch, R. E.; Zero viscosity limit for analytic solutions of the NavierStokes equation on a half-space. II. Construction of the Navier-Stokes solution. Comm. Math. Phys., 192(2):463-491, 1998.

[35] Schlichting, H. Boundary layer theory. Eighth edition. Springer-Verlag, Berlin, 2000. 
[36] Wang, C., Wang, Y., and Zhang, Z.; Zero-viscosity Limit of the Navier-Stokes equations in the analytic setting. Arch. Rational. Mech. Anal. 224 (2017) 555-595.

[37] Xin, Z., and Zhang., L.; On the global existence of solutions to the Prandtl's system. Adv. Math., 181:88-133, 2004. 